\title{
Intramolecular cycloaddition of azomethine ylides in the preparation of pyrrolidino- [2',3':3,4]pyrrolidino[1,2-a]benzimidazoles
}

\author{
Liping Meng, ${ }^{\dagger}$ James C. Fettinger, and Mark J. Kurth* \\ Department of Chemistry, University of California, One Shields Avenue, Davis, CA95616 \\ mjkurth@ucdavis.edu
}

\section{Supporting Information Table of Contents}

Experimental procedures for intermediates and selected library members:

Supporting Information Figure 1 plus associated text and references:

$$
\hookrightarrow \text {... }
$$

${ }^{1} \mathrm{H}$ NMR, ${ }^{13} \mathrm{C}$ NMR, HPLC/MS spectra of intermediates and selected library members: $\hookrightarrow$.

\footnotetext{
* To whom correspondence should be addressed. Fax: (530)752-8995. Tel: (530)752-8192.

${ }^{\dagger}$ On leave from the Pharmacy School of Tongji Medical College, Huangzhong University of Science \& Technology, Wuhan 430030, China
} 


\section{Experimental Procedures for Intermediates and Selected Library Members}

General Procedures. All chemicals were purchased from commercial suppliers and used without further purification. Analytical thin layer chromatography was carried out on pre-coated plates (silica gel $60 \mathrm{~F}_{254}, 250 \mu \mathrm{m}$ thickness) and visualized with UV light. Flash chromatography was performed using $60 \AA$ \&, $32-63 \mu \mathrm{m}$ silica gel (Scientific Adsorbents). Concentration refers to rotary evaporation under reduced pressure. ${ }^{1} \mathrm{H}$ NMR spectra were recorded at $300 \mathrm{MHz}$, or $600 \mathrm{MHz}$ at ambient temperature with $\mathrm{CDCl}_{3}$ as solvent. ${ }^{13} \mathrm{C} \mathrm{NMR}$ spectra were recorded at 75 $\mathrm{MHz}$, or $150 \mathrm{MHz}$ at ambient temperature with DMSO- $d_{6}$ or $\mathrm{CDCl}_{3}$ as solvent. Chemical shifts are reported in parts per million relative to $\mathrm{CDCl}_{3}\left({ }^{1} \mathrm{H}, \delta 7.26 ;{ }^{13} \mathrm{C}, \delta 77.16\right)$ or DMSO- $d_{6}\left({ }^{13} \mathrm{C}, \delta\right.$ 39.52). Infrared spectra were recorded on a FTIR spectrophotometer (Mattson Genesis II). The specifications of the LC/MS are as follows: electrospray (+) ionization, mass range $150-1500$ $\mathrm{Da}, 20 \mathrm{~V}$ cone voltage, and Xterra ${ }^{\circledR} \mathrm{MS} \mathrm{C} \mathrm{C}_{18}$ column $(2.1 \mathrm{~mm}$ x $50 \mathrm{~mm} \times 3.5 \mu \mathrm{m})$. In microwave mediated reactions, the temperature was maintained using Personal Chemistry Emrys Optimizer EXP microwave reactor, which heated the sealed samples to $130^{\circ} \mathrm{C}$ in $140 \mathrm{sec}$ and maintained that temperature for the duration of the 20 min reactions. HOBt refers to 1-hydroxybenzotriazole and EDC refers to $N$-ethyl- $N^{\prime}$-(3-dimethylaminopropyl)carbodiimide hydrochloride.

\section{General procedure for the synthesis of 2-diethoxymethyl-1H-benzimidazoles 2a-d.}

To a solution of sodium ethoxide $(1.20 \mathrm{~g}, 52.2 \mathrm{mmol} \mathrm{Na}$ in $30 \mathrm{~mL}$ dry ethanol) were added $o$ diaminoarene $(25.3 \mathrm{mmol})$ and ethyl diethoxyacetate $(5.35 \mathrm{~g}, 30.3 \mathrm{mmol})$. The mixture was refluxed for $24 \mathrm{~h}$, cooled to room temperature, and the solvent was removed under vacuum. The residue was dissolved in water, neutralized with acetic acid, and extracted with ethyl acetate. The combined organics were dried over anhydrous sodium sulfate, filtered, and evaporated to dryness. The residue was subjected to flash column chromatography $\left(\mathrm{CHCl}_{3} / \mathrm{EtOAc} \mathrm{1:1)}\right.$.

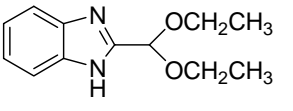

2-Diethoxymethyl-1H-benzimidazole $2 \mathrm{a}$ : yield $=82 \%$; white needles (recrystallization from EtOAc/n-hexane $1: 1), \operatorname{mp} 174-175^{\circ} \mathrm{C}$ (lit. $174-175^{\circ} \mathrm{C}$ from benzene) ${ }^{1}$ IR (neat) $v_{\max } 3327,3057,2887,1629,1593,1484,1448,1277,1070,744 \mathrm{~cm}^{-1} ;{ }^{1} \mathrm{H}$ NMR $(600$ $\left.\mathrm{MHz}, \mathrm{CDCl}_{3}\right), \delta 9.90(\mathrm{~s}, 1 \mathrm{H}), 7.78(\mathrm{~s}, 1 \mathrm{H}), 7.45(\mathrm{~s}, 1 \mathrm{H}), 7.25-7.26(\mathrm{~m}, 2 \mathrm{H}), 5.76(\mathrm{~s}, 1 \mathrm{H}), 3.64-3.76$ $(\mathrm{m}, 4 \mathrm{H}), 1.23(\mathrm{t}, 6 \mathrm{H}, J=7.2 \mathrm{~Hz}) ;{ }^{13} \mathrm{C}$ NMR $\left(150 \mathrm{MHz}, \mathrm{CDCl}_{3}\right) \delta 151.4,143.3,133.0,123.6$, 122.5, 120.4, 111.3, 97.2, 62.5, 15.3; ESIMS m/z 221.20 $(\mathrm{M}+\mathrm{H})^{+}$; Purity was determined to be $100 \%$ by HPLC analysis on the basis of absorption at $214 \mathrm{~nm}$.

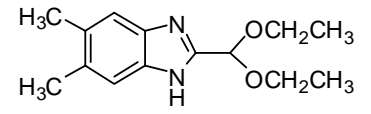

2-Diethoxymethyl-5,6-dimethyl-1H-benzimidazole $2 \mathbf{b}$ : yield $=48 \%$; white crystals (recrystallization from benzene) $\mathrm{mp} 111-112^{\circ} \mathrm{C}$; IR (neat) $v_{\max } 3283,3037,2881$, 1588, 1489, 1443, 1370, 1272, 1065, $858 \mathrm{~cm}^{-1} ;{ }^{1} \mathrm{H}$ NMR (600 MHz, $\left.\mathrm{CDCl}_{3}\right), \delta 9.32(\mathrm{~s}, 1 \mathrm{H}), 7.53$ $(\mathrm{s}, 1 \mathrm{H}), 7.21(\mathrm{~s}, 1 \mathrm{H}), 5.72(\mathrm{~s}, 1 \mathrm{H}), 3.63-3.75(\mathrm{~m}, 4 \mathrm{H}), 2.36(\mathrm{~s}, 6 \mathrm{H}), 1.25(\mathrm{t}, 6 \mathrm{H}, J=7.2 \mathrm{~Hz}) ;{ }^{13} \mathrm{C}$ NMR $\left(150 \mathrm{MHz}, \mathrm{CDCl}_{3}\right) \delta 150.5,142.0,132.8,131.4,128.6,120.3,111.4,97.2,62.4,20.7$,

\footnotetext{
${ }^{1}$ Vinot, N. S. Compt. Rend. 1961, 253, 2986.
} 
20.5, 15.4. ESIMS $m / z$ 249.12 $(\mathrm{M}+\mathrm{H})^{+}$; Purity was determined to be $98.8 \%$ by HPLC analysis on the basis of absorption at $214 \mathrm{~nm}$.

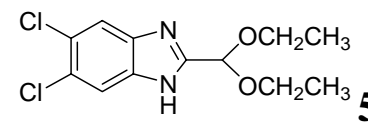

5,6-Dichloro-2-diethoxymethyl-1H-benzimidazole 2c: yield $=74 \%$; pink oil; IR (neat) $v_{\max } 3388,3093,2887,1577,1444,1289,1091,869,662 \mathrm{~cm}^{-1} ;{ }^{1} \mathrm{H}$ NMR (600 $\left.\mathrm{MHz}, \mathrm{CDCl}_{3}\right), \delta 7.71(\mathrm{~s}, 2 \mathrm{H}), 5.75(\mathrm{~s}, 1 \mathrm{H}), 3.60-3.74(\mathrm{~m}, 4 \mathrm{H}), 1.16(\mathrm{t}, 6 \mathrm{H}, J=7.2 \mathrm{~Hz}) ;{ }^{13} \mathrm{C}$ NMR $\left(150 \mathrm{MHz}, \mathrm{DMSO}-d_{6}\right) \delta 15.1,61.9,96.5,113.1,120.4,124.0,124.9,133.4,142.3,154.0$; ESIMS $\mathrm{m} / \mathrm{z} 290.99(\mathrm{M}+\mathrm{H})^{+}$; Purity was determined to be $99.1 \%$ by HPLC analysis on the basis of absorption at $214 \mathrm{~nm}$.

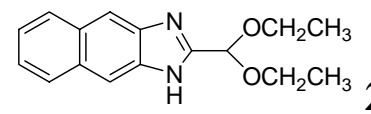

analytical data are in accord with literature values. ${ }^{2}$

\section{General procedure for the synthesis of 1-allyl-1H-benzimidazole-2-carbaldehydes 4a-d.}

2-(Diethoxymethyl)-1H-benzimidazole 2a-d $(7.6 \mathrm{mmol})$ was added to $\mathrm{NaH}(60 \%$ dispersion in mineral oil; $367 \mathrm{mg}, 9.2 \mathrm{mmol})$ in THF $(200 \mathrm{~mL})$ and refluxed for $30 \mathrm{~min}$. 3-Bromo-1-propene (1.38 g, $11.4 \mathrm{mmol})$ was added and the resulting solution refluxed for a further $8 \mathrm{~h}$. TLC of the reaction mixture showed the starting material was completely converted to product. The mixture was cooled to room temperature and water $(100 \mathrm{~mL})$ was added. The product was extracted with $\mathrm{Et}_{2} \mathrm{O}(2 \times 75 \mathrm{~mL})$, dried $\left(\mathrm{MgSO}_{4}\right)$, filtered, and evaporated to dryness to yield the crude 1-allyl2-(diethoxymethyl)-1H-benzimidazole (3a-d) as a brown viscous oil which, without purification, was subjected to the following deprotection step.

Water $(10 \mathrm{~mL})$ and $37 \%$ hydrochloride acid $(5 \mathrm{ml})$ were added to a THF $(30 \mathrm{~mL})$ solution of the crude 1-allyl-2-(diethoxymethyl)-1H-benzimidazole (3a-d) $(7.6 \mathrm{mmol})$. After refluxing for $24 \mathrm{~h}$, the reaction mixture was neutralized with saturated sodium bicarbonate and extracted with EtOAc $(3 \times 70 \mathrm{~mL})$. The extract was washed with brine $(2 \times 50 \mathrm{~mL})$, dried, filtered, and evaporated. The residue was chromatographed on silica gel (Hexane/EtOAc 2:1).

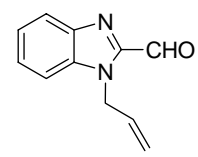

1-Allyl-1H-benzimidazole-2-carbaldehyde 4a: yield = 92\%. The analytical data are in accord with literature values. ${ }^{3}$

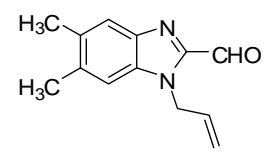

1-Allyl-5,6-dimethyl-1H-benzimidazole-2-carbaldehyde $\mathbf{4 b}$ : yield $=99 \%$; pale yellow solid $\mathrm{mp} 91-92^{\circ} \mathrm{C}$; IR (neat) $v_{\max } 3083,2866,1686,1572,1489,1438,1391 ; 1267,1019$, 915, 843, $781 \mathrm{~cm}^{-1} ;{ }^{1} \mathrm{H}$ NMR (600 MHz, $\left.\mathrm{CDCl}_{3}\right), \delta 10.04(\mathrm{~s}, 1 \mathrm{H}), 7.66(\mathrm{~s}, 1 \mathrm{H}), 7.19(\mathrm{~s}, 1 \mathrm{H})$,

\footnotetext{
${ }^{2}$ Nagashima, H.; Inoue, H.; Yoshioka, N. J. Phy. Chem. B 2004, 108, 6144.

${ }^{3}$ O'Shaughnessy, J.; Aldabbagh, F. Synthesis 2005, 1069.
} 
5.94-6.00 (m, 1H), 5.20-5.22 (m, 2H), 5.18 (br d, 1H, J=10.2 Hz), 4.99 (br d, 1H, J=10.2 Hz), $2.41(\mathrm{~s}, 3 \mathrm{H}), 2.39(\mathrm{~s}, 3 \mathrm{H}) ;{ }^{13} \mathrm{C} \mathrm{NMR}\left(150 \mathrm{MHz}, \mathrm{CDCl}_{3}\right) \delta 184.8,145.5,142.0,137.6,135.4$, 134.1, 132.4, 122.0, 117.6, 111.0, 47.0, 21.3, 20.7; ESIMS m/z 233.08 ( $\mathrm{M}+\mathrm{H}^{+}, \mathrm{M}=$ molecular mass of the corresponding hydrate aldehyde); Purity was determined to be $95.0 \%$ by HPLC analysis on the basis of absorption at $214 \mathrm{~nm}$.

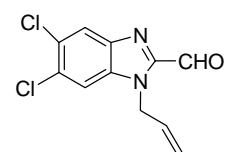

1-Allyl-5,6-dichloro-1H-benzimidazole-2-carbaldehyde 4c: yield $=52 \%$; pink

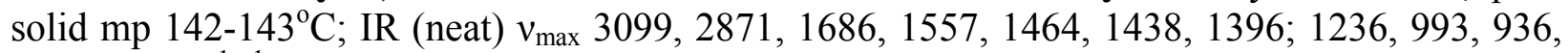
848, $796 \mathrm{~cm}^{-1}$; ${ }^{1} \mathrm{H}$ NMR $\left(600 \mathrm{MHz}, \mathrm{CDCl}_{3}\right), \delta 10.07(\mathrm{~s}, 1 \mathrm{H}), 8.01(\mathrm{~s}, 1 \mathrm{H}), 7.57(\mathrm{~s}, 1 \mathrm{H}), 5.92-5.98$ (m, 1H), 5.24 (br d, $1 \mathrm{H}, J=10.2 \mathrm{~Hz}), 5.20-5.21(\mathrm{~m}, 2 \mathrm{H}), 5.03(\mathrm{br} \mathrm{d}, 1 \mathrm{H}, J=10.2 \mathrm{~Hz}) ;{ }^{13} \mathrm{C} \mathrm{NMR}$ $\left(150 \mathrm{MHz}, \mathrm{CDCl}_{3}\right) \delta 184.6,147.2,142.0,135.5,131.6,131.5,129.0,123.6,118.6,112.7,47.4$; ESIMS $\mathrm{m} / \mathrm{z} 274.94\left(\mathrm{M}+\mathrm{H}^{+}, \mathrm{M}=\right.$ molecular mass of the corresponding hydrate aldehyde); Purity was determined to be $100 \%$ by HPLC analysis on the basis of absorption at $214 \mathrm{~nm}$.

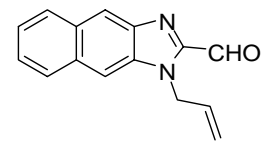

1-Allyl-1H-naphtho[2,3-d]imidazole-2-carbaldehyde $4 \mathrm{~d}$ : yield $=84 \%$; yellow solid mp 145-146 ${ }^{\circ}$; IR (neat) $v_{\max } 3047,2886,1696,1469,1422,1267,998,926,832,744 \mathrm{~cm}^{-1}$; ${ }^{1} \mathrm{H}$ NMR $\left(600 \mathrm{MHz}, \mathrm{CDCl}_{3}\right), \delta 10.20(\mathrm{~s}, 1 \mathrm{H}), 8.50(\mathrm{~s}, 1 \mathrm{H}), 8.03(\mathrm{~d}, 1 \mathrm{H}), 7.94(\mathrm{~d}, 1 \mathrm{H}), 7.85$ (s, 1H), 7.41-7.49 (m, 2H), 6.01-6.07 (m, 1H), 5.32-5.33 (m, 2H), 5.21 (br d, 1H, J=10.2 Hz), 5.08 (br d, $1 \mathrm{H}, J=10.2 \mathrm{~Hz}) ;{ }^{13} \mathrm{C}$ NMR $\left(150 \mathrm{MHz}, \mathrm{CDCl}_{3}\right) \delta 185.5,148.9,142.5,136.4,132.9,132.2$, 131.3, 129.1, 127.9, 126.1, 124.5, 120.5, 117.9, 107.2, 47.1; ESIMS m/z 255.06 ( $\mathrm{M}+\mathrm{H}^{+}, \mathrm{M}=$ molecular mass of the corresponding hydrate aldehyde); Purity was determined to be $96.1 \%$ by HPLC analysis on the basis of absorption at $214 \mathrm{~nm}$.

General procedure for the synthesis of ethyl pyrrolidino[2',3':3,4]pyrrolidino[1,2a]benzimidazole-2-carboxylic acid esters [Specific for 5aA-dA].

In a pyrex test tube, 1-allyl-1H-benzimidazole-2-carbaldehyde 4a-d (0.93 mmol), Nmethylglycine ethyl ester hydrochloride $(286 \mathrm{mg}, 1.86 \mathrm{mmol})$, and triethylamine $(185 \mathrm{mg}, 1.86$ mmol) in xylene $(3 \mathrm{~mL})$ were submitted to microwave irradiation at $130^{\circ} \mathrm{C}$ for $20 \mathrm{~min}$. The solvent was removed under vacuum and the residue was purified by flash column chromatography (EtOAc with 10\% EtOH).

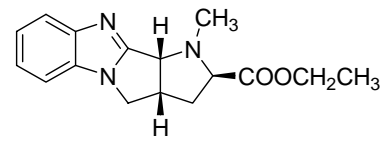

\section{Ethyl}

1-methyl-pyrrolidino[2',3':3,4]pyrrolidino[1,2-

a]benzimidazole-2-carboxylate 5aA: yield $=59 \%$; colorless crystals (recrystallization from EtOAc/n-hexane 1:1), mp 74-75 ${ }^{\circ} \mathrm{C}$; IR (neat) $v_{\max } 3062,2881,2793,1722,1618,1541,1458$, 1376, 1184, $734 \mathrm{~cm}^{-1}$; ${ }^{1} \mathrm{H}$ NMR $\left(600 \mathrm{MHz}, \mathrm{CDCl}_{3}\right), \delta 7.75(\mathrm{~m}, 1 \mathrm{H}), 7.20-7.27(\mathrm{~m}, 3 \mathrm{H}), 4.63(\mathrm{~d}$, $1 \mathrm{H}, J=7.8 \mathrm{~Hz}), 4.25(\mathrm{dd}, 1 \mathrm{H}, J=10.8 \mathrm{~Hz}, 8.4 \mathrm{~Hz}), 4.15(\mathrm{q}, 2 \mathrm{H}, J=7.2 \mathrm{~Hz}), 3.91(\mathrm{dd}, 1 \mathrm{H}, J=$ $10.8 \mathrm{~Hz}, 3 \mathrm{~Hz}), 3.79-3.85(\mathrm{~m}, 1 \mathrm{H}), 3.54(\mathrm{dd}, 1 \mathrm{H}, J=6.6 \mathrm{~Hz}, 4.8 \mathrm{~Hz}), 2.74(\mathrm{~s}, 3 \mathrm{H}), 2.38-2.42$ (m, $1 \mathrm{H}), 2.08-2.13(\mathrm{~m}, 1 \mathrm{H}), 1.23(\mathrm{t}, 3 \mathrm{H}, J=7.2 \mathrm{~Hz}) ;{ }^{13} \mathrm{C}$ NMR $\left(150 \mathrm{MHz}, \mathrm{CDCl}_{3}\right) \delta 172.8,158.3$, $147.1,131.4,123.2,123.1,120.0,110.3,67.1,64.0,61.1,49.7,44.2$, 37.4, 36.6, 14.6; ESIMS 
$\mathrm{m} / \mathrm{z} 286.04(\mathrm{M}+\mathrm{H})^{+}$; Purity was determined to be $96.7 \%$ by HPLC analysis on the basis of absorption at $214 \mathrm{~nm}$.

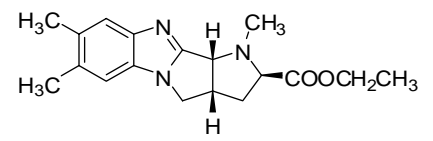

Ethyl 7,8-dimethyl-1-methyl-pyrrolidino[3,4-I]pyrrolidino[1,2a]benzimidazole-2-carboxylate 5bA: yield $=66 \%$; off-white solid $\mathrm{mp} \mathrm{mp} 144-146^{\circ} \mathrm{C}$; IR (neat) $v_{\max } 3026,2881,2793,1722,1531,1495,1469,1370,1179,1045,899,848,724 \mathrm{~cm}^{-1}$; ${ }^{1} \mathrm{H}$ NMR $\left(600 \mathrm{MHz}, \mathrm{CDCl}_{3}\right), \delta 7.54(\mathrm{~s}, 1 \mathrm{H}), 7.08(\mathrm{~s}, 1 \mathrm{H}), 4.65(\mathrm{~d}, 1 \mathrm{H}, J=7.8 \mathrm{~Hz}), 4.19-4.25(\mathrm{~m}, 3 \mathrm{H}), 3.88$ $(\mathrm{dd}, 1 \mathrm{H}, J=10.8 \mathrm{~Hz}, 2.4 \mathrm{~Hz}), 3.79-3.82(\mathrm{~m}, 1 \mathrm{H}), 3.54(\mathrm{dd}, 1 \mathrm{H}, J=6.0 \mathrm{~Hz}, 5.4 \mathrm{~Hz}), 2.79(\mathrm{~s}, 3 \mathrm{H})$, 2.41-2.46 (m, 1H), $2.35(\mathrm{~s}, 6 \mathrm{H}), 2.12-2.16(\mathrm{~m}, 1 \mathrm{H}), 1.28(\mathrm{t}, 3 \mathrm{H}, J=7.2 \mathrm{~Hz}) ;{ }^{13} \mathrm{C} \mathrm{NMR}(150$ $\left.\mathrm{MHz} \mathrm{CDCl}_{3}\right) \delta 172.9,157.9,147.8,131.6,131.1,130.6,120.5,110.2,66.8,63.9,61.0,49.3$, 44.0, 37.7, 36.5, 20.7, 20.6, 14.6; ESIMS m/z $314.13(\mathrm{M}+\mathrm{H})^{+}$; Purity was determined to be $96.1 \%$ by HPLC analysis on the basis of absorption at $214 \mathrm{~nm}$.<smiles>CCOC(=O)C1CC2CC3CN(C2)C3Nc2cc(Cl)c(Cl)cc21</smiles>

Ethyl 7,8-dichloro-1-methyl-pyrrolidino[2',3’:3,4]pyrrolidino[1,2a]benzimidazole-2-carboxylate 5cA: yield $=93 \%$; colorless crystals (recrystallization from EtOAc/n-hexane 1:1), mp 146-147 ${ }^{\circ}$; IR (neat) $v_{\max } 3104,2881,1728,1526,1453,1401,1199$, 1060, 899, 843, $801 \mathrm{~cm}^{-1}$; ${ }^{1} \mathrm{H}$ NMR $\left(300 \mathrm{MHz}, \mathrm{CDCl}_{3}\right), \delta 7.83(\mathrm{~s}, 1 \mathrm{H}), 7.41(\mathrm{~s}, 1 \mathrm{H}), 4.59(\mathrm{~d}, 1 \mathrm{H}$, $J=5.4 \mathrm{~Hz}), 4.19-4.29(\mathrm{~m}, 3 \mathrm{H}), 3.82-3.93(\mathrm{~m}, 2 \mathrm{H}), 3.64(\mathrm{dd}, 1 \mathrm{H}, J=6.9 \mathrm{~Hz}, 4.2 \mathrm{~Hz}), 2.78(\mathrm{~s}$, $3 \mathrm{H}), 2.42-2.51(\mathrm{~m}, 1 \mathrm{H}), 2.12-2.21(\mathrm{~m}, 1 \mathrm{H}), 1.31(\mathrm{t}, 3 \mathrm{H}, J=7.2 \mathrm{~Hz}) ;{ }^{13} \mathrm{C} \mathrm{NMR}(150 \mathrm{MHz}$, $\left.\mathrm{CDCl}_{3}\right) \delta 172.7,161.3,148.4,131.2,126.5,126.4,121.6,111.4,67.1,63.8,61.0,49.5,44.3$, 37.3, 36.4, 14.6; ESIMS m/z $355.94(\mathrm{M}+\mathrm{H})^{+}$; Purity was determined to be $93.9 \%$ by HPLC analysis on the basis of absorption at $214 \mathrm{~nm}$.

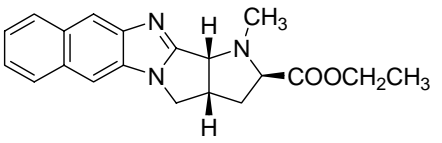

Ethyl 1-methylpyrrolidino[2',3':3,4]pyrrolidino[1,2a]naphtho[2,3-d]imidazole-2-carboxylate 5dA: yield = 52\%; off-white solid mp $143-144^{\circ} \mathrm{C}$; IR (neat) $v_{\max } 3052,2871,2793,1728,1536,1448,1407,1184,853,739 \mathrm{~cm}^{-1} ;{ }^{1} \mathrm{H}$ NMR $(600$ $\left.\mathrm{MHz}, \mathrm{CDCl}_{3}\right), \delta 8.22(\mathrm{~s}, 1 \mathrm{H}), 7.98-8.00(\mathrm{~m}, 1 \mathrm{H}), 7.91-7.92(\mathrm{~m}, 1 \mathrm{H}), 7.69(\mathrm{~s}, 1 \mathrm{H}), 7.38-7.43(\mathrm{~m}$, 2H), $4.67(\mathrm{~d}, 1 \mathrm{H}, J=7.8 \mathrm{~Hz}), 4.33(\mathrm{dd}, 1 \mathrm{H}, J=10.2 \mathrm{~Hz}, 8.4 \mathrm{~Hz}), 4.23(\mathrm{q}, 2 \mathrm{H}, J=7.2 \mathrm{~Hz}), 4.00$ $(\mathrm{dd}, 1 \mathrm{H}, J=10.2 \mathrm{~Hz}, 3.6 \mathrm{~Hz}), 3.87-3.93(\mathrm{~m}, 1 \mathrm{H}), 3.66(\mathrm{dd}, 1 \mathrm{H}, J=6.6 \mathrm{~Hz}, 4.2 \mathrm{~Hz}), 2.85(\mathrm{~s}, 3 \mathrm{H})$, 2.47-2.52 (m, 1H), 2.20-2.24 (m, 1H), $1.31(\mathrm{t}, 3 \mathrm{H}, J=7.2 \mathrm{~Hz}) ;{ }^{13} \mathrm{C} \mathrm{NMR}\left(150 \mathrm{MHz}, \mathrm{CDCl}_{3}\right) \delta$ $172.8,163.3,149.3,132.9,130.4,130.2$, 128.7, 127.5, 124.4, 123.6, 117.2, 105.6, 67.1, 63.9, 60.9, 49.2, 44.1, 37.4, 36.5, 14.5; ESIMS m/z 336.11 $(\mathrm{M}+\mathrm{H})^{+}$; Purity was determined to be $99.1 \%$ by HPLC analysis on the basis of absorption at $214 \mathrm{~nm}$.

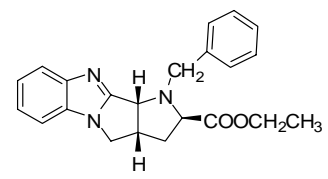

Ethyl 1-benzylpyrrolidino[2',3':3,4]pyrrolidino[1,2-a]benzimidazole-2carboxylate 5aB: yield $=43 \%$; colorless crystals (recrystallization from EtOAc/n-hexane 1:1), $\mathrm{mp} 128-129^{\circ} \mathrm{C}$; IR (neat) $v_{\max } 3057,2855,2819,1748,1500,1453,1381,1277,1200,744,703$ $\mathrm{cm}^{-1}$; ${ }^{1} \mathrm{H}$ NMR $\left(600 \mathrm{MHz}, \mathrm{CDCl}_{3}\right), \delta$ 7.71-7.23 (m, 1H), 7.11-7.28 (m, 8H), $4.69(\mathrm{~d}, 1 \mathrm{H}, J=7.8$ $\mathrm{Hz}), 4.42(\mathrm{~d}, 1 \mathrm{H}, J=13.2 \mathrm{~Hz}), 4.14$ (dd, $1 \mathrm{H}, J=10.2 \mathrm{~Hz}, 8.4 \mathrm{~Hz}), 4.00-4.10$ (m, 3H), 3.85 (dd, 
$1 \mathrm{H}, J=10.2 \mathrm{~Hz}, 3.0 \mathrm{~Hz}), 3.69-3.74(\mathrm{~m}, 1 \mathrm{H}), 3.48(\mathrm{dd}, 1 \mathrm{H}, J=7.2 \mathrm{~Hz}, 3.0 \mathrm{~Hz}), 2.31-2.35(\mathrm{~m}$, $1 \mathrm{H}), 2.05-2.10(\mathrm{~m}, 1 \mathrm{H}), 1.28(\mathrm{t}, 3 \mathrm{H}, J=6.6 \mathrm{~Hz}) ;{ }^{13} \mathrm{C} \mathrm{NMR}\left(150 \mathrm{MHz}, \mathrm{CDCl}_{3}\right) \delta 173.2,159.6$, 149.0, 138.5, 131.9, 129.4, 128.2, 127.2, 122.3, 122.1, 120.3, 109.9, 63.6, 62.4, 60.6, 53.3, 48.9, 43.7, 36.8, 14.4; ESIMS m/z $362.08(\mathrm{M}+\mathrm{H})^{+}$; Purity was determined to be $99.6 \%$ by HPLC analysis on the basis of absorption at $214 \mathrm{~nm}$.

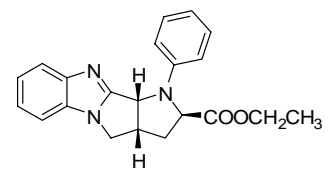

Ethyl 1-phenylpyrrolidino[2',3':3,4]pyrrolidino[1,2-a]benzimidazole-2carboxylate 5aC: yield $=10 \%$; white needles (recrystallization from $\mathrm{CH}_{3} \mathrm{CN} / \mathrm{H}_{2} \mathrm{O} 1: 2$ ), mp 188$189^{\circ} \mathrm{C}$; IR (neat) $v_{\max } 3057,2943,2092,1722,1598,1510,1453,1345,1262,1179,734,693$ $\mathrm{cm}^{-1}$; ${ }^{1} \mathrm{H}$ NMR $\left(600 \mathrm{MHz}, \mathrm{CDCl}_{3}\right), \delta$ 7.78-7.79 (m, 1H), 7.22-7.33 (m, 5H), 7.08-7.10 (m, 2H), 6.82-6.84 (m, 1H), $5.36(\mathrm{~d}, 1 \mathrm{H}, J=8.4 \mathrm{~Hz}), 4.53(\mathrm{~d}, 1 \mathrm{H}, J=7.8 \mathrm{~Hz}), 4.25(\mathrm{dd}, 1 \mathrm{H}, J=10.2 \mathrm{~Hz}$, $7.2 \mathrm{~Hz}$ ), 4.13-4.22 (m, 2H), 4.04 (br d, 1H, $J=10.2 \mathrm{~Hz}), 3.91-3.97(\mathrm{~m}, 1 \mathrm{H}), 2.53(\mathrm{dd}, 1 \mathrm{H}, J=$ $12.6 \mathrm{~Hz}, 7.8 \mathrm{~Hz}), 2.26-2.32(\mathrm{~m}, 1 \mathrm{H}), 1.21(\mathrm{t}, 3 \mathrm{H}, J=7.2 \mathrm{~Hz}) ;{ }^{13} \mathrm{C} \mathrm{NMR}\left(150 \mathrm{MHz}, \mathrm{CDCl}_{3}\right) \delta$ $173.8,159.5,149.0,145.9,132.1,129.6,122.8,122.5,121.0,118.8,113.9$, 109.9, 62.9, 61.4, 61.0, 46.2, 43.8, 35.8, 14.5; ESIMS $\mathrm{m} / \mathrm{z} 348.05(\mathrm{M}+\mathrm{H})^{+}$; Purity was determined to be $100 \%$ by HPLC analysis on the basis of absorption at $214 \mathrm{~nm}$.

\section{General procedure for EDC coupling to give pyrrolidino[2',3':3,4]pyrrolidino[1,2- a]benzimidazole-2-carboxamides.}

To a THF (10mL) solution of ethyl pyrrolidino[2',3':3,4]pyrrolidino[1,2-a]benzimidazole-2carboxylate $5 \mathbf{a A}-\mathbf{d A}(1.85 \mathrm{mmol})$ was added $5 \mathrm{M} \mathrm{NaOH}(4.8 \mathrm{~mL})$. The reaction mixture was stirred at $60^{\circ} \mathrm{C}$ for $3 \mathrm{~h}$. The reaction mixture was allowed to cool to room temperature, neutralized by concentrated $\mathrm{HCl}$ to $\mathrm{pH} 7$, and evaporated in vacuo to dryness. Addition of ethanol resulted in a white precipitate which was removed by filtration. The filtrate was evaporated in vacuo and the residue, crude pyrrolidino[2',3':3,4]pyrrolidino[1,2a]benzimidazole-2-carboxylic acid $\mathbf{6 a A - d A}$, was used in the next step without purification.

The pyrrolidino[2',3':3,4]pyrrolidino[1,2-a]benzimidazole-2-carboxylic acid 6aA-dA (0.44 mmol) was dissolved in DMF $(2 \mathrm{~mL})$ and THF $(1.5 \mathrm{~mL})$. Amine (1-12, $1.32 \mathrm{mmol})$, Nhydroxybenzotriazole $(89 \mathrm{mg}, 0.66 \mathrm{mmol})$, and triethylamine $(0.66 \mathrm{mmol})$ were added; the solution was cooled to $0{ }^{\circ} \mathrm{C}$ over $30 \mathrm{~min}$. EDC hydrochloride [ $N$-(3-dimethylaminopropyl)- $N$ ethylcarbodiimide hydrochloride. $0.66 \mathrm{mmol}$ ] was added and the reaction was allowed to warm to room temperature with stirring for $24 \mathrm{~h}$. The resulting reaction mixture was diluted with water $(15 \mathrm{~mL})$ and extracted with EtOAc $(3 \times 15 \mathrm{~mL})$. The combined organics were washed with aq. sodium bicarbonate, water, $1 \mathrm{~N}$ aq $\mathrm{HCl}$, and brine, dried over sodium sulfate, filtered, and concentrated to give the crude material. Purification by preparative HPLC delivered the pyrrolidino[2',3':3,4]pyrrolidino[1,2-a]imidazole-2-carboxamides.

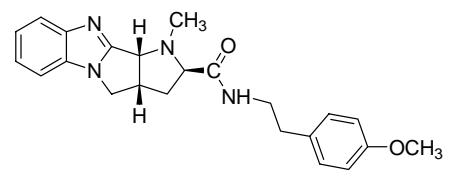

N-(4-Methoxyphenethyl)-1methylpyrrolidino[2',3’:3,4]pyrrolidino[1,2-a]benzimidazole-2-carboxamide 7aA\{1\}: yield 
$=78 \%$; colorless crystals (recrystallization from EtOAc/n-hexane $1: 1$ ), mp $172-173^{\circ} \mathrm{C}$; IR (neat) $v_{\max } 3404,3042,2834,1681,1613,1510,1453,1411,1184,812,750 \mathrm{~cm}^{-1} ;{ }^{1} \mathrm{H}$ NMR $(600 \mathrm{MHz}$, $\left.\mathrm{CDCl}_{3}\right), \delta$ 7.73-7.75 $(\mathrm{m}, 1 \mathrm{H}), 7.29-7.31(\mathrm{~m}, 1 \mathrm{H}), 7.22-7.26(\mathrm{~m}, 2 \mathrm{H}), 7.10-7.13(\mathrm{~m}, 2 \mathrm{H}), 6.83-$ $6.85(\mathrm{~m}, 2 \mathrm{H}), 6.78(\mathrm{br} \mathrm{s}, 1 \mathrm{H}, \mathrm{NH}), 4.72(\mathrm{~d}, 1 \mathrm{H}, J=7.2 \mathrm{~Hz}), 4.20$ (dd, $1 \mathrm{H}, J=10.2 \mathrm{~Hz}, 7.8 \mathrm{~Hz})$, $3.88(\mathrm{dd}, 1 \mathrm{H}, J=10.2 \mathrm{~Hz}, 3.0 \mathrm{~Hz}), 3.78(\mathrm{~s}, 3 \mathrm{H}), 3.58-3.63(\mathrm{~m}, 1 \mathrm{H}), 3.54-3.57(\mathrm{~m}, 1 \mathrm{H}), 3.44-$ $3.50(\mathrm{~m}, 1 \mathrm{H}), 3.06(\mathrm{dd}, 1 \mathrm{H}, J=10.2 \mathrm{~Hz}, 9.0 \mathrm{~Hz}), 2.77-2.80(\mathrm{dt}, 2 \mathrm{H}, J=7.2 \mathrm{~Hz}, 6.6 \mathrm{~Hz}), 2.58$ (s, 3H), 2.13-2.23 (m, 2H); ${ }^{13} \mathrm{C}$ NMR $\left(150 \mathrm{MHz}, \mathrm{CDCl}_{3}\right) \delta$ 172.5, 158.5, 157.6, 148.9, 132.1, $130.9,129.9,122.8,122.5,120.5,114.2,110.1,67.9,64.0,55.5,48.9,43.7,40.3,38.9,37.0$, 35.0; ESIMS $\mathrm{m} / \mathrm{z} 391.16(\mathrm{M}+\mathrm{H})^{+}$; Purity was determined to be $96.0 \%$ by HPLC analysis on the basis of absorption at $214 \mathrm{~nm}$.

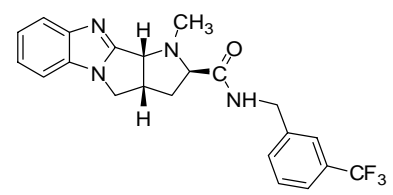

1-Methyl-2-(3-(trifluoromethyl)benzyl)pyrrolidino[2',3':3,4] pyrrolidino[1,2-a]benzimidazole-2-carboxamide $7 \mathbf{a A}\{2\}$ : yield $=89 \%$; colorless oil; ${ }^{1} \mathrm{H}$ NMR (600 MHz, $\mathrm{CDCl}_{3}$ ), $\delta$ 7.71-7.73 (m, 1H), 7.41-7.52 (m, 4H), 7.22-7.29 (m, 3H), 4.72 (br d, $1 \mathrm{H}, J$ $=7.2 \mathrm{~Hz}), 4.50(\mathrm{dd}, 1 \mathrm{H}, \mathrm{J}=15.6,6.6 \mathrm{~Hz}), 4.44(\mathrm{dd}, 1 \mathrm{H}, \mathrm{J}=15.6,6.6 \mathrm{~Hz}), 4.18(\mathrm{~m}, 1 \mathrm{H}), 3.87(\mathrm{br}$ d, $1 \mathrm{H}, J=10.2 \mathrm{~Hz}$ ), 3.58-3.64 (m, 1H), $3.14($ br t, $1 \mathrm{H}, J=8.1 \mathrm{~Hz}), 2.67(\mathrm{~s}, 3 \mathrm{H}), 2.25-2.30(\mathrm{~m}$, $1 \mathrm{H}), 2.18-2.22(\mathrm{~m}, 1 \mathrm{H}) ;{ }^{13} \mathrm{C} \mathrm{NMR}\left(150 \mathrm{MHz}, \mathrm{CDCl}_{3}\right) \delta 172.7,157.5,148.8,139.7,132.0,131.2$ (q), 131.0, 129.5, 124.5 (q), 124.4 (q), 124.2 (q), 122.8, 122.5, 120.3, 110.2, 67.8, 64.0, 49.0, 43.6, 42.6, 38.9, 37.0; ESIMS $\mathrm{m} / \mathrm{z} 415.18(\mathrm{M}+\mathrm{H})^{+}$; Purity was determined to be $93.2 \%$ by HPLC analysis on the basis of absorption at $214 \mathrm{~nm}$.

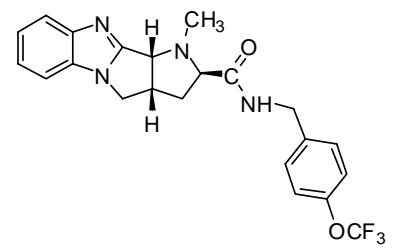

1-Methyl-N-(4-

(trifluoromethoxy)benzyl)pyrrolidino[2',3':3,4]pyrrolidino[1,2-a]benzimidazole-2-

carboxamide 7aA $\{3\}$ : yield $=65 \%$; off-white solid $\mathrm{mp} 131-133^{\circ} \mathrm{C}$; IR (neat) $v_{\max } 3290,3062$, $2824,1665,1505,1453,1272,822,734 \mathrm{~cm}^{-1}$; ${ }^{1} \mathrm{H}$ NMR $\left(600 \mathrm{MHz}, \mathrm{CDCl}_{3}\right), \delta$ 7.74-7.75 (m, 1H), 7.18-7.32 (m, 7H), 4.76 (d, $1 \mathrm{H}, J=7.2 \mathrm{~Hz}), 4.43-4.51(\mathrm{~m}, 2 \mathrm{H}), 4.21(\mathrm{brt}, 1 \mathrm{H}, J=9.0 \mathrm{~Hz}), 3.87$ $(\mathrm{t}, 1 \mathrm{H}, J=9.0 \mathrm{~Hz}), 3.66(\mathrm{~m}, 1 \mathrm{H}), 3.18(\mathrm{t}, 1 \mathrm{H}, J=7.8 \mathrm{~Hz}), 2.70(\mathrm{~s}, 3 \mathrm{H}), 2.24-2.32(\mathrm{~m}, 2 \mathrm{H}) ;{ }^{13} \mathrm{C}$ NMR $\left(150 \mathrm{MHz}, \mathrm{CDCl}_{3}\right) \delta 172.5,157.3,148.7,148.6,137.2,131.9,129.2,128.5,122.7,122.4$, 121.4, 121.3, 121.5 (q), 120.3, 110.1, 67.7, 64.0, 48.8, 43.5, 42.3, 38.8, 37.0; ESIMS m/z 431.03 $(\mathrm{M}+\mathrm{H})^{+}$; Purity was determined to be $93.8 \%$ by HPLC analysis on the basis of absorption at $214 \mathrm{~nm}$.

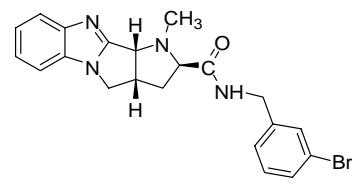

N-(3-Bromobenzyl)-1-methylpyrrolidino[2',3':3,4]pyrrolidino[1,2-

a]benzimidazole-2-carboxamide 7aA $\{4\}$ : yield $=65 \%$; off-white solid $\mathrm{mp} 50-51^{\circ} \mathrm{C}$; IR (neat) $v_{\max } 3264,3057,2804,1660,1515,1453,1215,739,698 \mathrm{~cm}^{-1} ;{ }^{1} \mathrm{H}$ NMR $\left(600 \mathrm{MHz}, \mathrm{CDCl}_{3}\right), \delta$ 7.73-7.75 (m, 1H), 7.19-7.40 (m, 6H), $4.75(\mathrm{~d}, 1 \mathrm{H}, J=7.2 \mathrm{~Hz}), 4.45(\mathrm{dd}, 1 \mathrm{H}, \mathrm{J}=15.0 \mathrm{~Hz}, 6.6$ $\mathrm{Hz}), 4.39$ (dd, 1H, J = 15.0 Hz, $5.4 \mathrm{~Hz}), 4.20(\mathrm{dd}, 1 \mathrm{H}, J=10.8 \mathrm{~Hz}, 7.8 \mathrm{~Hz}), 3.89$ (dd, $1 \mathrm{H}, J=$ 
$10.8 \mathrm{~Hz}, 3.0 \mathrm{~Hz}$ ), 3.64-3.67 (m, 1H), $3.15(\mathrm{dd}, 1 \mathrm{H}, J=8.4 \mathrm{~Hz}, 8.4 \mathrm{~Hz}), 2.68(\mathrm{~s}, 3 \mathrm{H}), 2.22-2.31$ $(\mathrm{m}, 2 \mathrm{H}) ;{ }^{13} \mathrm{C}$ NMR $\left(150 \mathrm{MHz}, \mathrm{CDCl}_{3}\right) \delta 172.6,157.4,148.7,140.9,132.0,130.9,130.8,130.6$, $126.5,122.9,122.8,122.6,120.4,110.2,67.8,64.0,49.0,43.6,42.5,38.9,37.1$; ESIMS m/z $426.98(\mathrm{M}+\mathrm{H})^{+}$; Purity was determined to be $97.2 \%$ by HPLC analysis on the basis of absorption at $214 \mathrm{~nm}$.

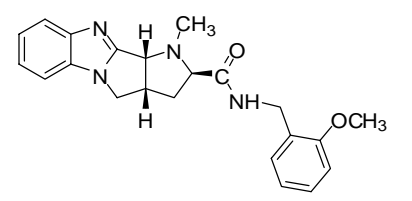

N-(2-Methoxybenzyl)-1-methylpyrrolidino[2',3':3,4]pyrrolidino[1,2a]benzimidazole-2-carboxamide 7aA $\{6\}$ : yield $=85 \%$; pale brown oil; ${ }^{1} \mathrm{H}$ NMR $(300 \mathrm{MHz}$, $\left.\mathrm{CDCl}_{3}\right), \delta$ 7.75-7.78 (m, 1H), 7.19-7.34 (m, 5H), 6.89-6.96 (m, 2H), $4.77(\mathrm{~d}, 1 \mathrm{H}, J=7.5 \mathrm{~Hz})$, 4.43-4.51 (m, 2H), $4.21(\mathrm{dd}, 1 \mathrm{H}, J=10.5 \mathrm{~Hz}, 7.8 \mathrm{~Hz}), 3.89(\mathrm{dd}, 1 \mathrm{H}, J=10.5 \mathrm{~Hz}, 3.0 \mathrm{~Hz}), 3.87$ (s, $1 \mathrm{H}), 3.62-3.70(\mathrm{~m}, 1 \mathrm{H}), 3.12(\mathrm{t}, 1 \mathrm{H}, J=7.5 \mathrm{~Hz}), 2.67(\mathrm{~s}, 3 \mathrm{H}), 2.20-2.35(\mathrm{~m}, 2 \mathrm{H}) ;{ }^{13} \mathrm{C}$ NMR $\left(75 \mathrm{MHz}, \mathrm{CDCl}_{3}\right) \delta 172.0,157.6,157.5,148.7,131.9,129.8,129.1,126.1,122.6,122.3,120.7$, 120.2, 110.4, 110.0, 67.8, 63.8, 55.4, 48.8, 43.5, 39.1, 38.7, 36.7; ESIMS m/z 377.12 (M + H) ${ }^{+}$; Purity was determined to be $94.2 \%$ by HPLC analysis on the basis of absorption at $214 \mathrm{~nm}$.

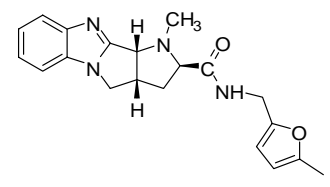

1-Methyl-N-((5-methylfuran-2yl)methyl)pyrrolidino[2',3':3,4]pyrrolidino[1,2-a]benzimidazole-2-carboxamide 7 aA $\{8\}$ : yield $=86 \%$; dark orange oil; ${ }^{1} \mathrm{H}$ NMR $\left(600 \mathrm{MHz}, \mathrm{CDCl}_{3}\right), \delta$ 7.74-7.76 $(\mathrm{m}, 1 \mathrm{H}), 7.30-7.32(\mathrm{~m}$, 1H), 7.24-7.26 (m, 2H), 7.03 (br s, 1H, NH), 6.09 (d, $1 \mathrm{H}, J=3.0 \mathrm{~Hz}), 5.89(\mathrm{~m}, 1 \mathrm{H}), 4.78(\mathrm{~d}, 1 \mathrm{H}$, $J=6.6 \mathrm{~Hz}), 4.42(\mathrm{dd}, 1 \mathrm{H}, \mathrm{J}=15 \mathrm{~Hz}, 5.4 \mathrm{~Hz}), 4.37(\mathrm{dd}, 1 \mathrm{H}, \mathrm{J}=15 \mathrm{~Hz}, 5.4 \mathrm{Hx}), 4.22(\mathrm{dd}, 1 \mathrm{H}, J=$ $10.8 \mathrm{~Hz}, 7.8 \mathrm{~Hz}), 3.91(\mathrm{dd}, 1 \mathrm{H}, J=10.8 \mathrm{~Hz}, 3.0 \mathrm{~Hz}$ ), 3.64-3.69 (m, $1 \mathrm{H}), 3.13(\mathrm{dd}, 1 \mathrm{H}, J=7.8$ $\mathrm{Hz}, 7.8 \mathrm{~Hz}), 2.69(\mathrm{~s}, 3 \mathrm{H}), 2.22-2.32(\mathrm{~m}, 2 \mathrm{H}), 2.26(\mathrm{~s}, 3 \mathrm{H}) ;{ }^{13} \mathrm{C} \mathrm{NMR}\left(150 \mathrm{MHz}, \mathrm{CDCl}_{3}\right) \delta 172.3$, 157.6, 152.3, 149.4, 148.9, 132.1, 122.8, 122.5, 120.5, 110.1, 108.4, 106.5, 67.8, 64.0, 49.0, 43.6, 38.9, 37.0, 36.4, 13.8; ESIMS $\mathrm{m} / \mathrm{z} 351.09(\mathrm{M}+\mathrm{H})^{+}$; Purity was determined to be $90.1 \%$ by HPLC analysis on the basis of absorption at $214 \mathrm{~nm}$.

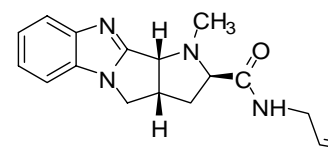

= N-Allyl-1-methylpyrrolidino[2',3':3,4]pyrrolidino[1,2-a]benzimidazole2-carboxamide 7aA $\{10\}$ : yield $=63 \%$; off-white solid $\mathrm{mp} 107-109^{\circ} \mathrm{C}$; IR (neat) $v_{\max } 3280,3062$, $2830,1650,1515,1448,1215,926,734 \mathrm{~cm}^{-1} ;{ }^{1} \mathrm{H}$ NMR $\left(300 \mathrm{MHz}, \mathrm{CDCl}_{3}\right), \delta$ 7.73-7.77 (m, $\left.1 \mathrm{H}\right)$, 7.22-7.34 (m, 3H), $6.92(\mathrm{br} \mathrm{s}, 1 \mathrm{H}, \mathrm{NH}), 5.79-5.92(\mathrm{~m}, 1 \mathrm{H}), 5.14-5.24(\mathrm{~m}, 2 \mathrm{H}), 4.80(\mathrm{~d}, 1 \mathrm{H}, J=$ $7.2 \mathrm{~Hz}), 4.24(\mathrm{dd}, 1 \mathrm{H}, J=10.5 \mathrm{~Hz}, 7.8 \mathrm{~Hz}), 3.86-3.96(\mathrm{~m}, 3 \mathrm{H}), 3.63-3.73(\mathrm{~m}, 1 \mathrm{H}), 3.13(\mathrm{dd}, 1 \mathrm{H}$, $J=8.1 \mathrm{~Hz}, 8.1 \mathrm{~Hz}), 2.72(\mathrm{~s}, 3 \mathrm{H}), 2.22-2.36(\mathrm{~m}, 2 \mathrm{H}) ;{ }^{13} \mathrm{C} \mathrm{NMR}\left(75 \mathrm{MHz}, \mathrm{CDCl}_{3}\right) \delta 172.3$, 157.5, 148.8, 134.2, 132.0, 122.7, 122.4, 120.3, 116.6, 110.0, 67.8, 64.0, 48.8, 43.6, 41.4, 38.9, 37.0; ESIMS $\mathrm{m} / \mathrm{z} 297.13(\mathrm{M}+\mathrm{H})^{+}$; Purity was determined to be $91.3 \%$ by HPLC analysis on the basis of absorption at $214 \mathrm{~nm}$. 


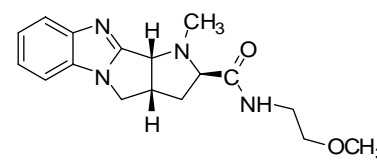

N-(2-Methoxyethyl)-1-methylpyrrolidino[2',3':3,4]pyrrolidino[1,2-

a]benzimidazole-2-carboxamide $7 \mathbf{7 a A}\{11\}$ : yield $=58 \%$; off-white solid $\mathrm{mp} 112-114^{\circ} \mathrm{C} ;{ }^{1} \mathrm{H}$ NMR $\left(300 \mathrm{MHz}, \mathrm{CDCl}_{3}\right), \delta$ 7.75-7.79 (d, $\left.1 \mathrm{H}\right), 7.24-7.35(\mathrm{~m}, 3 \mathrm{H}), 7.04(\mathrm{br} \mathrm{s}, 1 \mathrm{H}, \mathrm{NH}), 4.82(\mathrm{~d}$, $1 \mathrm{H}, J=7.2 \mathrm{~Hz}), 4.26(\mathrm{dd}, 1 \mathrm{H}, J=10.8 \mathrm{~Hz}, 8.1 \mathrm{~Hz}), 3.94(\mathrm{dd}, 1 \mathrm{H}, J=10.8 \mathrm{~Hz}, 3.3 \mathrm{~Hz}$ ), 3.64$3.75(\mathrm{~m}, 1 \mathrm{H}), 3.41-3.55(\mathrm{~m}, 4 \mathrm{H}), 3.38(\mathrm{~s}, 3 \mathrm{H}), 3.15(\mathrm{dd}, 1 \mathrm{H}, J=8.4 \mathrm{~Hz}, 7.5 \mathrm{~Hz}), 2.72(\mathrm{~s}, 3 \mathrm{H})$, 2.21-2.36 (m, 2H); ${ }^{13} \mathrm{C}$ NMR $\left(150 \mathrm{MHz}, \mathrm{CDCl}_{3}\right) \delta 172.5,157.6,148.7,131.9,122.6,122.3$, $120.3,110.0,71.3,67.7,63.9,58.9,48.9,43.4,38.9,38.7,36.7$; ESIMS $\mathrm{m} / \mathrm{z} 315.14(\mathrm{M}+\mathrm{H})^{+}$; Purity was determined to be $96.2 \%$ by HPLC analysis on the basis of absorption at $214 \mathrm{~nm}$.

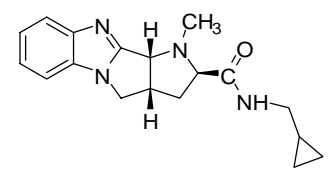

N-(Cyclopropylmethyl)-1-methylpyrrolidino[2',3':3,4]pyrrolidino[1,2a]benzimidazole-2-carboxamide 7aA $\{12\}$ : yield $=61 \%$; pale yellow oil; ${ }^{1} \mathrm{H}$ NMR $(600 \mathrm{MHz}$, $\left.\mathrm{CDCl}_{3}\right), \delta$ 7.70-7.72 (m, 1H), 7.18-7.27 (m, 3H), $6.79($ br s, $1 \mathrm{H}, \mathrm{NH}), 4.77(\mathrm{~d}, 1 \mathrm{H}, J=7.2 \mathrm{~Hz})$, 4.19 (dd, $1 \mathrm{H}, J=10.2 \mathrm{~Hz}, 7.8 \mathrm{~Hz}), 3.87$ (dd, $1 \mathrm{H}, J=10.2 \mathrm{~Hz}, 3.6 \mathrm{~Hz}$ ), 3.62-3.66 (m, 1H), 3.02$3.15(\mathrm{~m}, 3 \mathrm{H}), 2.67(\mathrm{~s}, 3 \mathrm{H}), 2.18-2.27(\mathrm{~m}, 2 \mathrm{H}), 0.88-0.94(\mathrm{~m}, 1 \mathrm{H}), 0.45-0.49(\mathrm{~m}, 2 \mathrm{H}), 0.15-0.17$ $(\mathrm{m}, 2 \mathrm{H}) ;{ }^{13} \mathrm{C}$ NMR $\left(150 \mathrm{MHz}, \mathrm{CDCl}_{3}\right) \delta 172.3,157.5,148.7,132.0,122.8,122.6,120.4,110.2$, 67.9, 64.1, 49.0, 43.9, 43.6, 39.0, 37.0, 11.0, 3.57, 3.55; ESIMS m/z $311.16(\mathrm{M}+\mathrm{H})^{+}$; Purity was determined to be $97.5 \%$ by HPLC analysis on the basis of absorption at $214 \mathrm{~nm}$.

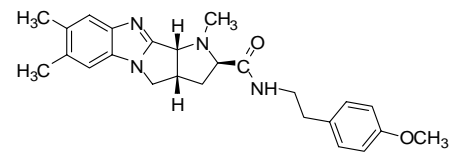

1,7,8-Trimethyl-N-(4methoxyphenethyl)pyrrolidino[2',3':3,4]pyrrolidino[1,2-a]benzimidazole-2-carboxamide 7bA $\{1\}$ : yield $=81 \%$; off-white solid mp $132-134^{\circ} \mathrm{C} ;{ }^{1} \mathrm{H}$ NMR $\left(600 \mathrm{MHz}, \mathrm{CDCl}_{3}\right), \delta 7.51(\mathrm{~s}$, $1 \mathrm{H}), 7.12(\mathrm{~d}, 2 \mathrm{H}, J=7.8 \mathrm{~Hz}$ ), $7.09(\mathrm{~s}, 1 \mathrm{H}), 6.84(\mathrm{~d}, 2 \mathrm{H}, J=7.8 \mathrm{~Hz}), 6.81$ (br s, $1 \mathrm{H}, \mathrm{NH}), 4.71$ $(\mathrm{d}, 1 \mathrm{H}, J=6.6 \mathrm{~Hz}), 4.21(\mathrm{t}, 1 \mathrm{H}, J=10.2 \mathrm{~Hz}), 3.84(\mathrm{dd}, 1 \mathrm{H}, J=10.2 \mathrm{~Hz}), 3.79(\mathrm{~s}, 3 \mathrm{H}), 3.53-3.62$ (m, 2H), 3.46-3.50 (m, 1H), $3.05(\mathrm{dd}, 1 \mathrm{H}, J=8.4 \mathrm{~Hz}, 7.2 \mathrm{~Hz}), 2.76-2.82(\mathrm{~m}, 2 \mathrm{H}), 2.56(\mathrm{~s}, 3 \mathrm{H})$, $2.36(\mathrm{~s}, 6 \mathrm{H}), 2.14-2.24(\mathrm{~m}, 2 \mathrm{H}) ;{ }^{13} \mathrm{C}$ NMR $\left(150 \mathrm{MHz}, \mathrm{CDCl}_{3}\right) \delta 172.5,158.5,156.6,147.2$, 132.0, 131.5, 130.9, 130.5, 129.9, 120.4, 114.2, 110.4, 67.9, 63.9, 55.5, 48.9, 43.8, 40.3, 38.9, 37.0, 35.0, 20.7, 20.6; ESIMS $\mathrm{m} / \mathrm{z} 419.15(\mathrm{M}+\mathrm{H})^{+}$; Purity was determined to be $94.3 \%$ by HPLC analysis on the basis of absorption at $214 \mathrm{~nm}$.

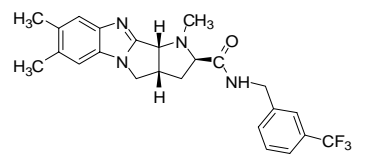

1,7,8-Trimethyl-2-(3-

(trifluoromethyl)benzyl)pyrrolidino[2',3':3,4]pyrrolidino[1,2-a]benzimidazole-2-

carboxamide $\mathbf{7 b A}\{2\}$ : yield $=59 \%$; white solid $\mathrm{mp} 122-123^{\circ} \mathrm{C}$; IR (neat) $v_{\max } 3290,2969,2814$, $1660,1520,1448,1329,1164,1076,848,750,698 \mathrm{~cm}^{-1} ;{ }^{1} \mathrm{H}$ NMR $\left(600 \mathrm{MHz}, \mathrm{CDCl}_{3}\right), \delta$ 7.44$7.54(\mathrm{~m}, 5 \mathrm{H}), 7.27$ (br s, 1H, NH), $7.09(\mathrm{~s}, 1 \mathrm{H}), 4.75(\mathrm{~d}, 1 \mathrm{H}, J=6.6 \mathrm{~Hz}), 4.55(\mathrm{dd}, 1 \mathrm{H}, J=15.0$ $\mathrm{Hz}, 6.6 \mathrm{~Hz}), 4.49(\mathrm{dd}, 1 \mathrm{H}, \mathrm{J}=15.0 \mathrm{~Hz}, 6.4 \mathrm{~Hz}), 4.18(\mathrm{dd}, 1 \mathrm{H}, J=10.8 \mathrm{~Hz}, 7.8 \mathrm{~Hz}), 3.86(\mathrm{dd}$, $1 \mathrm{H}, J=10.8 \mathrm{~Hz}, 3.0 \mathrm{~Hz}), 3.62-3.66(\mathrm{~m}, 1 \mathrm{H}), 3.17(\mathrm{dd}, 1 \mathrm{H}, J=7.8 \mathrm{~Hz}, 7.8 \mathrm{~Hz}), 2.67(\mathrm{~s}, 3 \mathrm{H})$, $2.35(\mathrm{~s}, 3 \mathrm{H}), 2.36(\mathrm{~s}, \mathrm{H}), 2.27(\mathrm{dd} 1 \mathrm{H}, \mathrm{J}=7.8 \mathrm{~Hz}, 6.6 \mathrm{~Hz}) ;{ }^{13} \mathrm{C}$ NMR $\left(150 \mathrm{MHz}, \mathrm{CDCl}_{3}\right) \delta \delta$ $172.8,156.5,147.4,139.7,132.0,131.5$ (q), 131.4, 129.5, 124.5 (q), 124.4 (q), 124.2 (q), 122.8, 
122.6, 120.4, 110.4, 67.9, 64.0, 48.9, 43.8, 42.6, 38.9, 37.2, 20.7, 20.6; ESIMS m/z 443.11 (M + $\mathrm{H})^{+}$; Purity was determined to be $97.3 \%$ by HPLC analysis on the basis of absorption at $214 \mathrm{~nm}$.

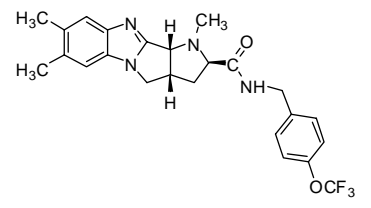

(trifluoromethoxy)benzyl)pyrrolidino[2',3':3,4]pyrrolidino[1,2-a]benzimidazole-2-

1,7,8-Trimethyl-N-(4carboxamide 7bA $\{3\}$ : yield $=87 \%$; white solid $\mathrm{mp} 180-182^{\circ} \mathrm{C} ;{ }^{1} \mathrm{H}$ NMR $\left(600 \mathrm{MHz}, \mathrm{CDCl}_{3}\right), \delta$ $7.51(\mathrm{~s}, 1 \mathrm{H}), 7.30-7.31(\mathrm{~m}, 2 \mathrm{H}), 7.18-7.22(\mathrm{~m}, 3 \mathrm{H}), 7.09(\mathrm{~s}, 1 \mathrm{H}, \mathrm{NH}), 4.75(\mathrm{~d}, 1 \mathrm{H}, J=7.2 \mathrm{~Hz})$, 4.49 (dd, $1 \mathrm{H}, J=15.0 \mathrm{~Hz}, 6.0 \mathrm{~Hz}$ ), 4.44 (dd, $1 \mathrm{H}, J=15 \mathrm{~Hz}, 5.4 \mathrm{~Hz}$ ), 4.19 (dd, $1 \mathrm{H}, J=10.8 \mathrm{~Hz}$, $7.8 \mathrm{~Hz}$ ), 3.87 (dd, $1 \mathrm{H}, J=10.8 \mathrm{~Hz}, 3.0 \mathrm{~Hz}), 3.66(\mathrm{~m}, 1 \mathrm{H}), 3.17$ (dd, $1 \mathrm{H}, J=7.8 \mathrm{~Hz}, 7.8 \mathrm{~Hz}), 2.68$ $(\mathrm{s}, 3 \mathrm{H}), 2.37(\mathrm{~s} 3 \mathrm{H}), 2.36(\mathrm{~s}, 3 \mathrm{H}), 2.27-2.29(\mathrm{~m}, 2 \mathrm{H}) ;{ }^{13} \mathrm{C} \mathrm{NMR}\left(150 \mathrm{MHz}, \mathrm{CDCl}_{3}\right) \delta 172.7$, 156.6, 148.8, 147.5, 137.4, 132.0, 131.4, 130.6, 129.4, 129.3, 121.5 (q), 120.5, 110.4, 67.9, 64.0, $48.9,43.7,42.4,39.0,37.2,20.7,20.6$; ESIMS $\mathrm{m} / \mathrm{z} 459.10(\mathrm{M}+\mathrm{H})^{+}$; Purity was determined to be $94.3 \%$ by HPLC analysis on the basis of absorption at $214 \mathrm{~nm}$.

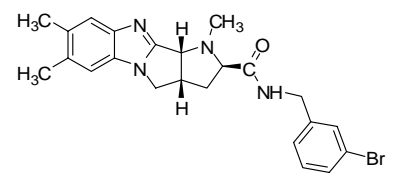

bromobenzyl)pyrrolidino[2',3':3,4]pyrrolidino[1,2-a]benzimidazole-2-carboxamide

1,7,8-Trimethyl-N-(37bA $\{4\}$ : yield $=85 \%$; yellow oil; ${ }^{1} \mathrm{H}$ NMR $\left(600 \mathrm{MHz}, \mathrm{CDCl}_{3}\right), \delta 7.50(\mathrm{~s}, 1 \mathrm{H}), 7.39-7.41(\mathrm{~m}$, 2H), 7.20-7.21 (m, 3H), 7.09 (s, 1H), 4.74 (d, $1 \mathrm{H}, J=7.8 \mathrm{~Hz}), 4.46$ (dd, $1 \mathrm{H}, J=15 \mathrm{~Hz}, 6.6 \mathrm{~Hz})$, $4.40(\mathrm{dd}, 1 \mathrm{H}, \mathrm{J}=15 \mathrm{~Hz}, 6.0 \mathrm{~Hz}), 4.18(\mathrm{dd}, 1 \mathrm{H}, J=10.2 \mathrm{~Hz}, 7.8 \mathrm{~Hz}), 3.86(\mathrm{dd}, 1 \mathrm{H}, J=10.2 \mathrm{~Hz}$, $3.6 \mathrm{~Hz}$ ), 3.62-3.67 (m, 1H), $3.15(\mathrm{dd}, 1 \mathrm{H}, J=7.8 \mathrm{~Hz}, 7.8 \mathrm{~Hz}), 2.66(\mathrm{~s}, 3 \mathrm{H}), 2.36(\mathrm{~s}, 3 \mathrm{H}), 2.35(\mathrm{~s}$, $3 \mathrm{H}), 2.27(\mathrm{dd}, 2 \mathrm{H}, J=7.8 \mathrm{~Hz}, 7.8 \mathrm{~Hz}) ;{ }^{13} \mathrm{C}$ NMR $\left(150 \mathrm{MHz}, \mathrm{CDCl}_{3}\right) \delta 172.7,156.6,147.4$, $140.9,132.0,131.4,130.9,130.6,126.5,123.0,120.4,110.4,67.9,64.0,48.9,43.8,42.5,39.0$, 37.2, 20.7, 20.6; ESIMS $\mathrm{m} / \mathrm{z} 454.98(\mathrm{M}+\mathrm{H})^{+}$; Purity was determined to be $91.2 \%$ by HPLC analysis on the basis of absorption at $214 \mathrm{~nm}$.

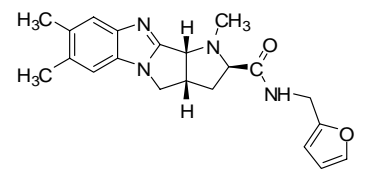

1,7,8-Trimethyl-N-(furan-2ylmethyl)pyrrolidino[2',3':3,4]pyrrolidino[1,2-a]benzimidazole-2-carboxamid 7bA\{7\}: yield $=77 \%$; off-white solid mp $189-190^{\circ} \mathrm{C} ;{ }^{1} \mathrm{H}$ NMR $\left(600 \mathrm{MHz}, \mathrm{CDCl}_{3}\right), \delta 7.40(\mathrm{~s}, 1 \mathrm{H}), 7.14(\mathrm{~s}, 1 \mathrm{H})$, $7.01($ br s, $1 \mathrm{H}, \mathrm{NH}), 6.98(\mathrm{~s}, 1 \mathrm{H}), 6.22(\mathrm{br} \mathrm{s}, 1 \mathrm{H}), 6.11(\mathrm{br} \mathrm{d}, 1 \mathrm{H}, J=2.4 \mathrm{~Hz}), 4.65(\mathrm{~d}, 1 \mathrm{H}, J=$ $7.2 \mathrm{~Hz}), 4.32-4.39(\mathrm{~m}, 2 \mathrm{H}), 4.06(\mathrm{dd}, 1 \mathrm{H}, J=9.1 \mathrm{~Hz}, 8.9 \mathrm{~Hz}), 3.75(\mathrm{~d}, 1 \mathrm{H}, J=10.2 \mathrm{~Hz}), 3.52-$ $3.54(\mathrm{~m}, 1 \mathrm{H}), 3.03(\mathrm{dd}, 1 \mathrm{H}, J=7.8 \mathrm{~Hz}, 7.8 \mathrm{~Hz}), 2.55(\mathrm{~s}, 3 \mathrm{H}), 2.25$ (s, 3H), 2.24 (s, 3H), 2.13$2.16(\mathrm{~m}, 1 \mathrm{H}) ;{ }^{13} \mathrm{C}$ NMR $\left(150 \mathrm{MHz}, \mathrm{CDCl}_{3}\right) \delta 172.4,156.6,151.3,147.4,142.5,131.9,131.3$, 130.5, 120.4, 110.6, 110.3, 107.5, 67.8, 63.9, 48.8, 43.6, 38.8, 37.0, 36.1, 20.6, 20.5; ESIMS m/z $365.12(\mathrm{M}+\mathrm{H})^{+}$; Purity was determined to be $97.0 \%$ by HPLC analysis on the basis of absorption at $214 \mathrm{~nm}$. 


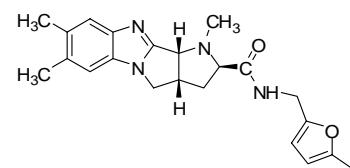

1,7,8-Trimethyl-N-((5-methylfuran-2yl)methyl)pyrrolidino[2',3':3,4]pyrrolidino[1,2-a]benzimidazole-2-carboxamide $7 \mathrm{bA}\{8\}$ : yield $=73 \%$; pale yellow solid mp $116-118^{\circ} \mathrm{C} ;{ }^{1} \mathrm{H}$ NMR $\left(600 \mathrm{MHz}, \mathrm{CDCl}_{3}\right), \delta 7.51(\mathrm{~s}, 1 \mathrm{H}), 7.09$ (s, 1H), 7.05 (br s, 1H, NH), $6.10(\mathrm{~d}, 1 \mathrm{H}, J=3.0 \mathrm{~Hz}), 5.90(\mathrm{~d}, 1 \mathrm{H}, J=3.0 \mathrm{~Hz}), 4.77(\mathrm{~d}, 1 \mathrm{H}, J=$ $7.2 \mathrm{~Hz}$ ), $4.43(\mathrm{dd}, 1 \mathrm{H}, J=15.0 \mathrm{~Hz}, 6.0 \mathrm{~Hz}), 4.38(\mathrm{dd}, 1 \mathrm{H}, \mathrm{J}=15.0 \mathrm{~Hz}, 5.4 \mathrm{~Hz}), 4.19$ (dd, 1H, $J=$ $10.2 \mathrm{~Hz}, 7.8 \mathrm{~Hz}$ ), 3.87 (dd, $1 \mathrm{H}, J=10.2 \mathrm{~Hz}, 3.0 \mathrm{~Hz}$ ), 3.64-3.69 (m, 1H), 3.14 (dd, $1 \mathrm{H}, J=7.2$ $\mathrm{Hz}, 7.2 \mathrm{~Hz}), 2.67(\mathrm{~s}, 3 \mathrm{H}), 2.37(\mathrm{~s}, 3 \mathrm{H}), 2.36(\mathrm{~s}, 3 \mathrm{H}), 2.24-2.31(\mathrm{~m}, 5 \mathrm{H}) ;{ }^{13} \mathrm{C} \mathrm{NMR}(150 \mathrm{MHz}$, $\left.\mathrm{CDCl}_{3}\right) \delta 172.4,156.7,152.2,149.5,147.3,132.0,131.4,130.5,120.4,110.3,108.4,106.5,67.9$, 64.0, 48.9, 43.7, 38.9, 37.0, 36.4, 20.7, 20.6, 13.8; ESIMS $\mathrm{m} / \mathrm{z} 379.15(\mathrm{M}+\mathrm{H})^{+}$; Purity was determined to be $90.1 \%$ by HPLC analysis on the basis of absorption at $214 \mathrm{~nm}$.

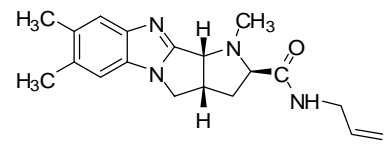

N-Allyl-1,7,8-trimethylpyrrolidino[2',3':3,4]pyrrolidino[1,2-

a]benzimidazole-2-carboxamide $\mathbf{7 b A}\{\mathbf{1 0}\}$ : yield $=83 \%$; white solid $\mathrm{mp} 184-185^{\circ} \mathrm{C}$; IR (neat) $v_{\max } 3290,2974,2804,1655,1515,1448,1360,926,838 \mathrm{~cm}^{-1}$; ${ }^{1} \mathrm{H}$ NMR $\left(600 \mathrm{MHz}, \mathrm{CDCl}_{3}\right), \delta$ $7.52(\mathrm{~s}, 1 \mathrm{H}), 7.10(\mathrm{~s}, 1 \mathrm{H}), 6.93(\mathrm{br} \mathrm{s}, 1 \mathrm{H}, \mathrm{NH}), 5.85-5.86(\mathrm{~m}, 1 \mathrm{H}), 5.15-5.22(\mathrm{~m}, 2 \mathrm{H}), 4.78(\mathrm{~d}$, $1 \mathrm{H}, J=5.4 \mathrm{~Hz}), 4.18(\mathrm{br} \mathrm{s}, 1 \mathrm{H}), 3.87-3.96(\mathrm{~m}, 3 \mathrm{H}), 3.66(\mathrm{br} \mathrm{s}, 1 \mathrm{H}), 3.14(\mathrm{br} \mathrm{s}, 1 \mathrm{H}), 2.70(\mathrm{~s}, 3 \mathrm{H})$, $2.37(\mathrm{~s}, 6 \mathrm{H}), 2.24-2.30(\mathrm{~m}, 2 \mathrm{H}) ;{ }^{13} \mathrm{C}$ NMR $\left(150 \mathrm{MHz}, \mathrm{CDCl}_{3}\right) \delta 172.4,156.6,147.4,134.3$, 131.9, 131.3, 130.5, 120.4, 116.6, 110.3, 67.9, 63.9, 48.8, 43.7, 41.4, 38.9, 37.1, 20.6, 20.5; ESIMS $\mathrm{m} / \mathrm{z} 325.12(\mathrm{M}+\mathrm{H})^{+}$; Purity was determined to be $98.9 \%$ by HPLC analysis on the basis of absorption at $214 \mathrm{~nm}$.

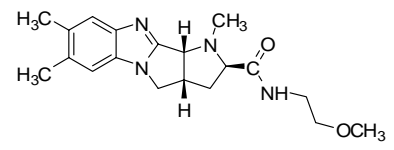

methoxyethyl)pyrrolidino[2',3':3,4]pyrrolidino[1,2-a]benzimidazole-2-carboxamide

1,7,8-Trimethyl-N-(27bA $\{11\}$ : yield $=64 \%$; white solid mp $196-198^{\circ} \mathrm{C} ;{ }^{1} \mathrm{H}$ NMR $\left(600 \mathrm{MHz}, \mathrm{CDCl}_{3}\right), \delta 7.52(\mathrm{~s}, 1 \mathrm{H})$, 7.10 (s, 1H), 7.07 (br s, 1H, NH), $4.78(\mathrm{~d}, 1 \mathrm{H}, J=6.6 \mathrm{~Hz}), 4.18$ (dd, $1 \mathrm{H}, J=9.6 \mathrm{~Hz}, 9.6 \mathrm{~Hz})$, 3.86 (br d, $1 \mathrm{H}, J=9.6 \mathrm{~Hz}), 3.63-3.68(\mathrm{~m}, 1 \mathrm{H}), 3.40-3.54(\mathrm{~m}, 4 \mathrm{H}), 3.38(\mathrm{~s}, 3 \mathrm{H}), 3.11(\mathrm{dd}, 1 \mathrm{H}, J=$ $7.8 \mathrm{~Hz}, 7.8 \mathrm{~Hz}), 2.68(\mathrm{~s}, 3 \mathrm{H}), 2.36(\mathrm{~s}, 6 \mathrm{H}), 2.22-2.30(\mathrm{~m}, 2 \mathrm{H}) ;{ }^{13} \mathrm{C} \mathrm{NMR}\left(150 \mathrm{MHz}, \mathrm{CDCl}_{3}\right) \delta$ 172.7, 156.8, 147.4, 131.9, 131.3, 130.6, 120.4, 110.3, 71.5, 67.9, 64.0, 59.0, 49.0, 43.7, 39.0, 38.9, 36.9, 20.7, 20.6; ESIMS $\mathrm{m} / \mathrm{z} 343.13(\mathrm{M}+\mathrm{H})^{+}$; Purity was determined to be $94.0 \%$ by HPLC analysis on the basis of absorption at $214 \mathrm{~nm}$.

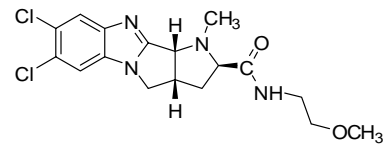

7,8-Dichloro-N-(2-methoxyethyl)-1methylpyrrolidino[2',3':3,4]pyrrolidino[1,2-a]benzimidazole-2-carboxamide 7cA\{11\}: yield $=83 \%$; yellow solid $\mathrm{mp} 114-116^{\circ} \mathrm{C}$; IR (neat) $v_{\max } 3316,3047,2840,1665,1541,1450,1401$, 1096, 832, $801 \mathrm{~cm}^{-1} ;{ }^{1} \mathrm{H}$ NMR (600 MHz, $\mathrm{CDCl}_{3}$ ), $\delta 7.85$ (s, $\left.1 \mathrm{H}\right), 7.43$ (s, 1H), 7.11 (br s, $1 \mathrm{H}$, $\mathrm{NH}), 4.89(\mathrm{~d}, 1 \mathrm{H}, J=6.6 \mathrm{~Hz}), 4.25(\mathrm{dd}, 1 \mathrm{H}, \mathrm{J}=9.0 \mathrm{~Hz}, 9.0 \mathrm{~Hz}), 3.92(\mathrm{~d}, 1 \mathrm{H}, \mathrm{J}=9.0 \mathrm{~Hz}), 3.78-$ $3.84(\mathrm{~m}, 1 \mathrm{H}), 3.40-3.50(\mathrm{~m}, 4 \mathrm{H}), 3.35(\mathrm{~s}, 3 \mathrm{H}), 3.10-3.12(\mathrm{dd}, 1 \mathrm{H}, J=7.8 \mathrm{~Hz}, 7.8 \mathrm{~Hz}), 2.71(\mathrm{~s}$, $3 \mathrm{H}), 2.32-2.37(\mathrm{~m}, 1 \mathrm{H}), 2.22-2.28(\mathrm{~m}, 1 \mathrm{H}) ;{ }^{13} \mathrm{C} \mathrm{NMR}\left(150 \mathrm{MHz}, \mathrm{CDCl}_{3}\right) \delta$ 171.3, 158.7, 146.3, 
130.6, 127.4, 127.3, 121.0, 111.7, 71.1, 67.9, 64.1, 58.9, 49.5, 43.7, 38.9, 38.1, 36.5; ESIMS m/z $384.99(\mathrm{M}+\mathrm{H})^{+}$; Purity was determined to be $92.4 \%$ by HPLC analysis on the basis of absorption at $214 \mathrm{~nm}$.

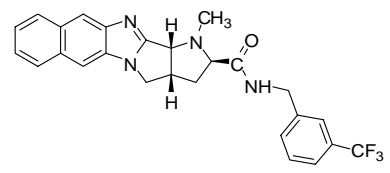

1-Methyl-2-(3-

(trifluoromethyl)benzyl)pyrrolidino[2',3':3,4]pyrrolidino[1,2-a]naphtho[2,3-d]imidazole-2carboxamide 7dA $\{2\}$ : yield $=80 \%$; yellow solid mp $141-142^{\circ} \mathrm{C} ;{ }^{1} \mathrm{H} \mathrm{NMR}\left(600 \mathrm{MHz}, \mathrm{CDCl}_{3}\right), \delta$ $8.21(\mathrm{~s}, 1 \mathrm{H}), 7.98(\mathrm{~d}, 1 \mathrm{H}, J=7.8 \mathrm{~Hz}), 7.9(\mathrm{~d}, 1 \mathrm{H}, J=7.8 \mathrm{~Hz}), 7.69(\mathrm{~s}, 1 \mathrm{H}), 7.39-7.56(\mathrm{~m}, 6 \mathrm{H})$, 7.29 (br s, $1 \mathrm{H}, \mathrm{NH}), 4.81(\mathrm{~d}, 1 \mathrm{H}, J=7.2 \mathrm{~Hz}), 4.57(\mathrm{dd}, 1 \mathrm{H}, J=15.0 \mathrm{~Hz}, 6.6 \mathrm{~Hz}), 4.51(\mathrm{dd}, 1 \mathrm{H}, \mathrm{J}$ $=15.0 \mathrm{~Hz}, 5.4 \mathrm{~Hz}), 4.26(\mathrm{dd}, 1 \mathrm{H}, J=10.2 \mathrm{~Hz}, 7.8 \mathrm{~Hz}), 3.97(\mathrm{~m}, 1 \mathrm{H}), 3.62-3.66(\mathrm{~m}, 1 \mathrm{H}), 3.25$ $(\mathrm{dd}, 1 \mathrm{H}, J=8.4 \mathrm{~Hz}, 8.4 \mathrm{~Hz}), 2.74(\mathrm{~s}, 3 \mathrm{H}), 2.30-2.36(\mathrm{~m}, 2 \mathrm{H}) ;{ }^{13} \mathrm{C}$ NMR $\left(150 \mathrm{MHz}, \mathrm{CDCl}_{3}\right) \delta$ 172.6, 161.7, 148.8, 139.5, 132.6, 131.2 (q), 131.1, 130.4, 130.3, 129.4, 128.7, 127.5, 125.0, 124.7 (q), 124.6 (q), 124.4 (q), 123.8, 117.2, 105.9, 67.8, 64.0, 48.8, 43.6, 42.6, 38.8, 37.1; ESIMS $\mathrm{m} / \mathrm{z} 465.10(\mathrm{M}+\mathrm{H})^{+}$; Purity was determined to be $96.4 \%$ by HPLC analysis on the basis of absorption at $214 \mathrm{~nm}$.

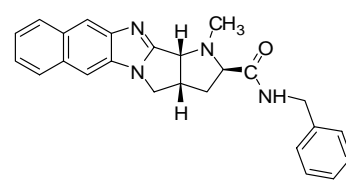

N-Benzyl-1-methylpyrrolidino[2',3':3,4]pyrrolidino[1,2-a]naphtho[2,3d]imidazole-2-carboxamide 7dA $\mathbf{5}\}$ : yield $=65 \%$; yellow oil; ${ }^{1} \mathrm{H}$ NMR $\left(300 \mathrm{MHz}, \mathrm{CDCl}_{3}\right), \delta$ $8.12(\mathrm{~s}, 1 \mathrm{H}), 7.83-7.92(\mathrm{~m}, 2 \mathrm{H}), 7.59(\mathrm{~s}, 1 \mathrm{H}), 7.18-7.38(\mathrm{~m}, 7 \mathrm{H}), 7.08$ (br s, $1 \mathrm{H}, \mathrm{NH}), 4.69(\mathrm{~d}$, $1 \mathrm{H}, J=7.2 \mathrm{~Hz}), 4.40(\mathrm{~d}, 2 \mathrm{H}, \mathrm{J}=2.7 \mathrm{~Hz}), 4.14(\mathrm{dd}, 1 \mathrm{H}, J=10.2 \mathrm{~Hz}, 7.8 \mathrm{~Hz}), 3.84(\mathrm{dd}, 1 \mathrm{H}, J=$ $10.2 \mathrm{~Hz}, 3.0 \mathrm{~Hz}), 3.52-3.64(\mathrm{~m}, 1 \mathrm{H}), 3.15(\mathrm{dd}, 1 \mathrm{H}, J=7.8 \mathrm{~Hz}, 7.8 \mathrm{~Hz}), 2.66$ (s, 3H), 2.20-2.30 $(\mathrm{m}, 2 \mathrm{H}) ;{ }^{13} \mathrm{C}$ NMR $\left(75 \mathrm{MHz}, \mathrm{CDCl}_{3}\right) \delta 172.4,162.0,149.0,138.5,132.7,130.5,130.3,129.0$, 128.8, 128.0, 127.8, 127.6, 124.7, 123.9, 117.3, 105.9, 68.0, 64.2, 49.0, 43.6, 43.3, 39.0, 37.1; ESIMS $\mathrm{m} / \mathrm{z} 397.09(\mathrm{M}+\mathrm{H})^{+}$; Purity was determined to be $91.7 \%$ by HPLC analysis on the basis of absorption at $214 \mathrm{~nm}$. 


\section{Supporting Information - Figure 1}

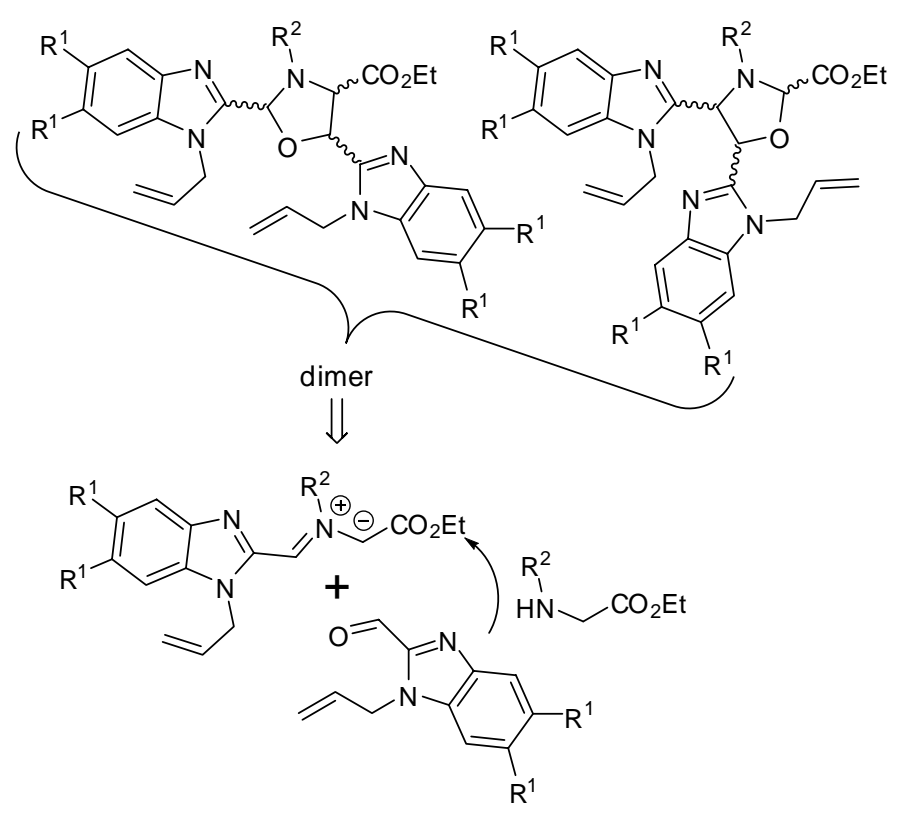

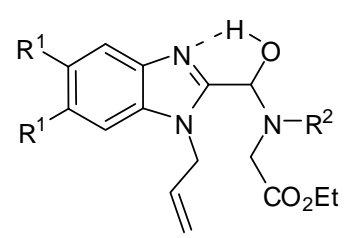

hemiaminal

Dimer formation occurs when the azomethine ylide undergoes an intermolecular addition to its precursor aldehyde (e.g., 4a-d) producing ethyl 3-(substituted)oxazolidine-4-carboxylates as a complex mixture of regio- and stereoisomers. The data, consisting of crude LC trace and LCMS as well as ${ }^{1} \mathrm{H}-\mathrm{NMR}$, supporting this conclusion are depicted on pages S14-S18 [(1)crude LC trace on page S14, (2) Dimer LC-MS on page S15, (3) Dimer ${ }^{1}$ H-NMR on page S16, (4) Hemiaminal LC-MS on page S17, and (5) Hemiaminal ${ }^{1}$ H-NMR on page S18]. Dimer formation is documented in the literature examples noted in manuscript reference 8 and reproduced here:

(8) (a) Brome, V. A.; Harwood, L. M.; Osborn, H. M. I. Can. J. Chem. 2006, 84, 1448. (b) Aldous, D. J.; Drew, M. G. B.; Draffin, W. N.; Hamelin, E. M.-N.; Harwood, L. M.; Thurairatnam, S. Synthesis, 2005, 3271. (c) Travnicek, M.; Potacek, M. ARKIVOC (Gainesville, FL, U. S.) 2001, 156. (d) Rudas, M.; Fejes, I.; Nyerges, M.; Szollosy, A.; Toke, L.; Groundwater, P. W. J. Chem. Soc., Perkin Trans. 1 1999, 1167. (e) Felluga, F.; Pitacco, G.; Visintin, C.; Valentin, E. Helv. Chim. Acta 1997, 80, 1457. (f) Kanemasa, S.; Sakamoto, K.; Tsuge, O. Bull. Chem. Soc. Jpn. 1989, 62, 1960. 
(1) Crude LC trace from $4 a+N$-Bn-Gly-OEt.

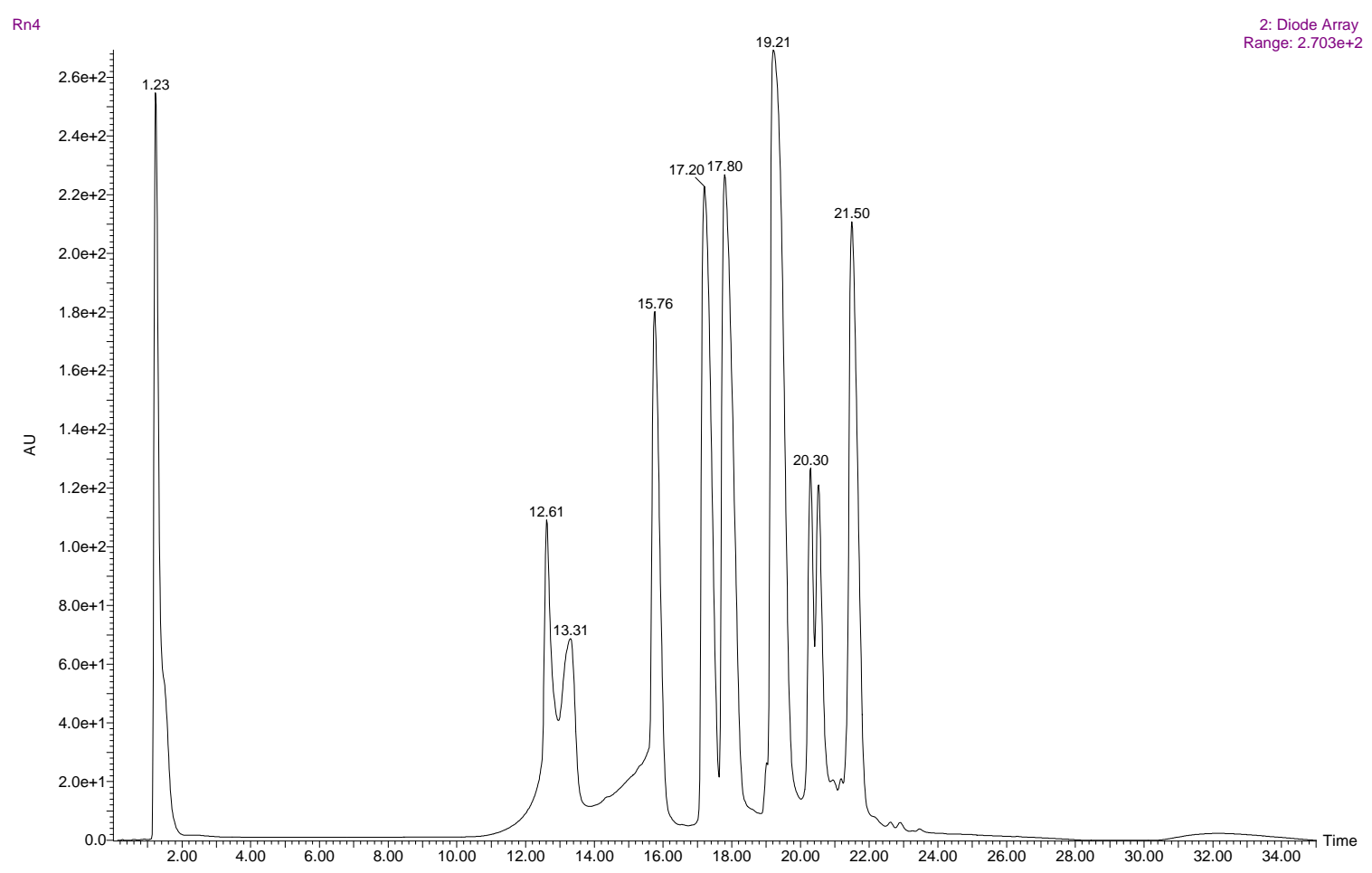

Peak 15.76: hemiaminal by-product from $\mathbf{4 a}+N$-Bn-Gly-OEt

Peak 17.80: desired product 5aB from $\mathbf{4 a}+N$-Bn-Gly-OEt

Peaks 17.20, 19.21, 20.30 (both peaks), and 21.50: dimer by-products from $4 \mathbf{4 a}+N$-Bn-Gly-OEt 
(2) Dimer LC-MS from 4a $+N$-Bn-Gly-OEt (dimer peak 19.21 from Crude LC trace).

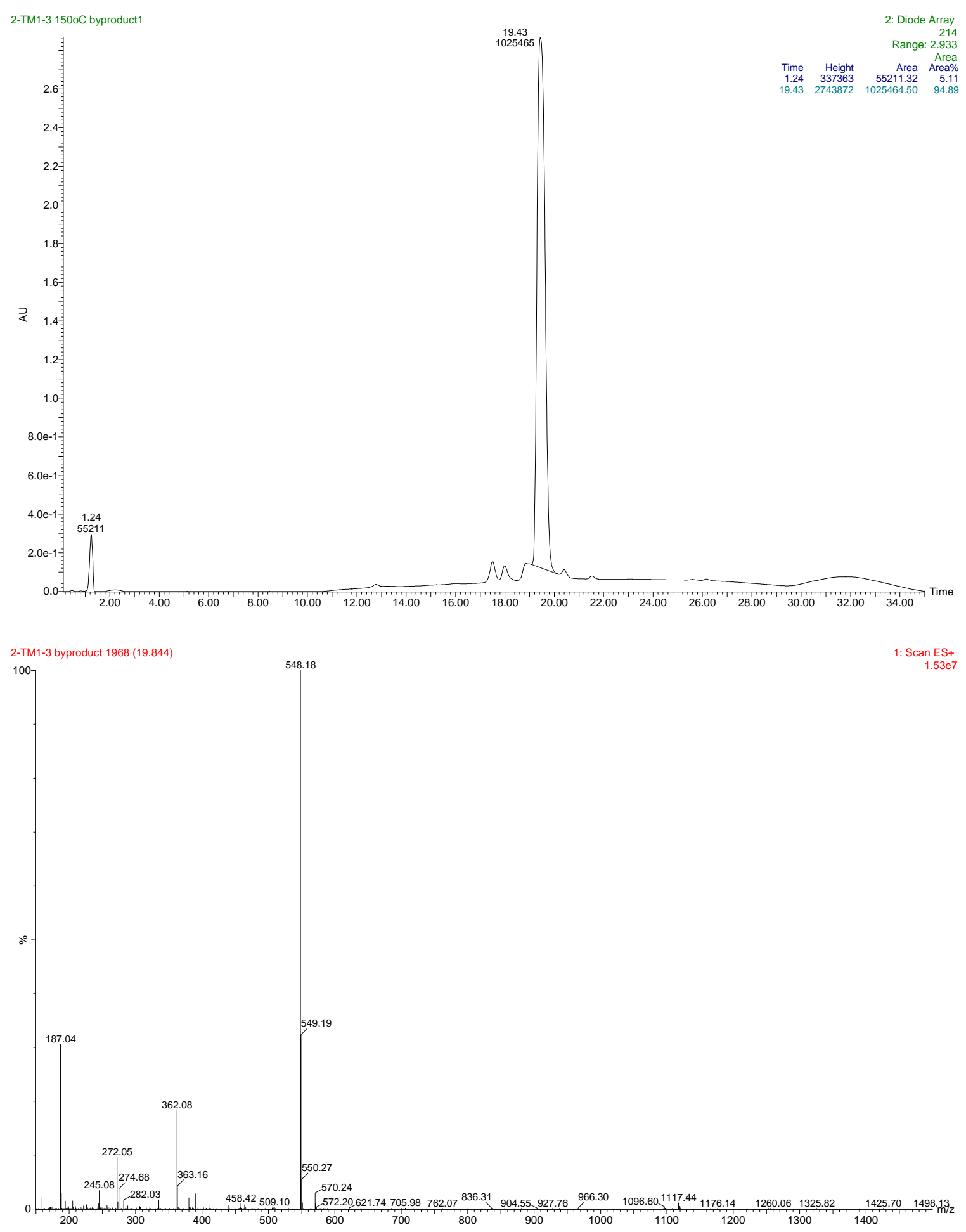


(3) Dimer ${ }^{1} \mathbf{H}$-NMR from 4a $+N$-Bn-Gly-OEt (dimer peak 19.21 from Crude LC trace).

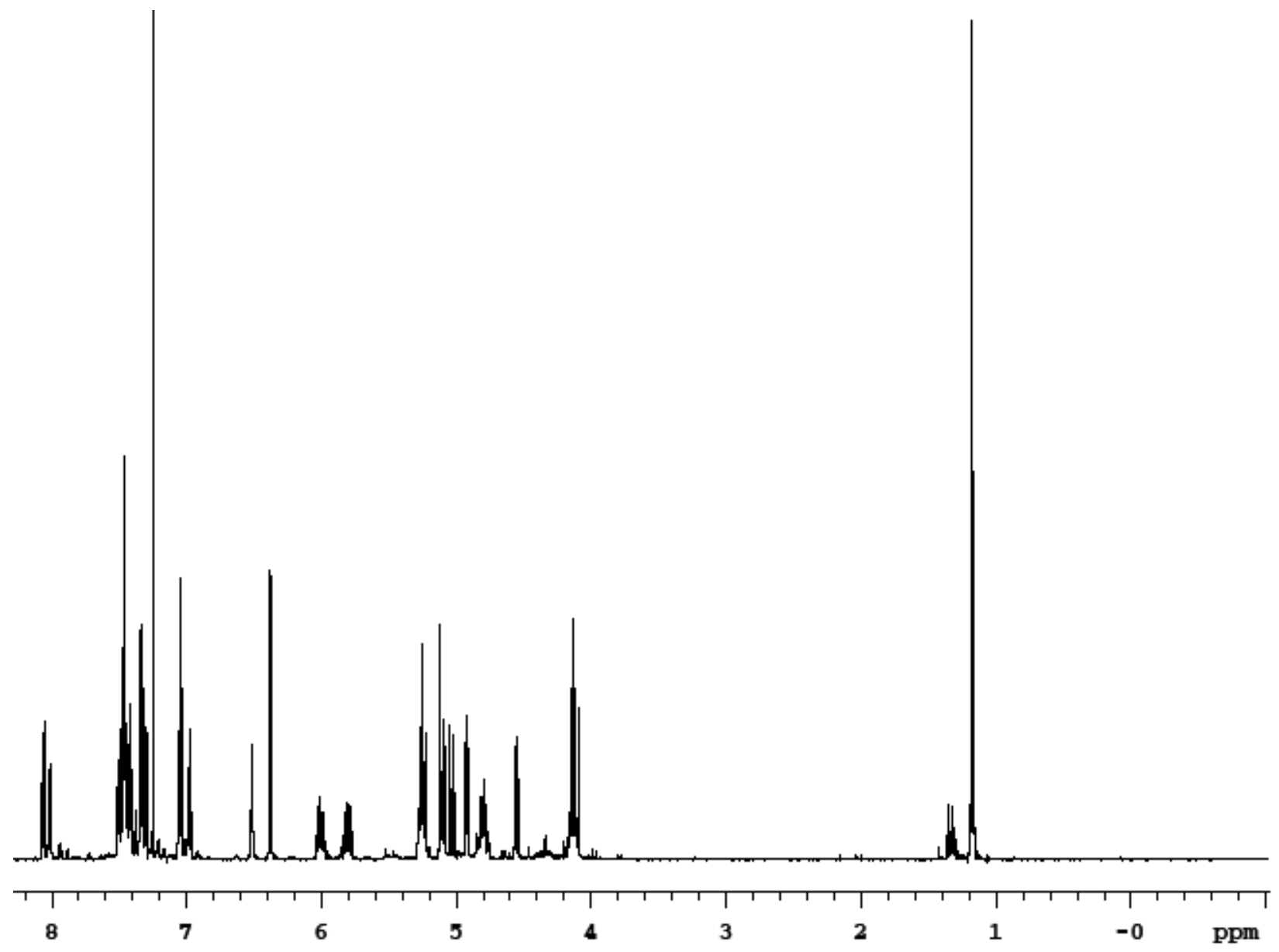


(4) Hemiaminal LC-MS from 4a $+N$-Bn-Gly-OEt.

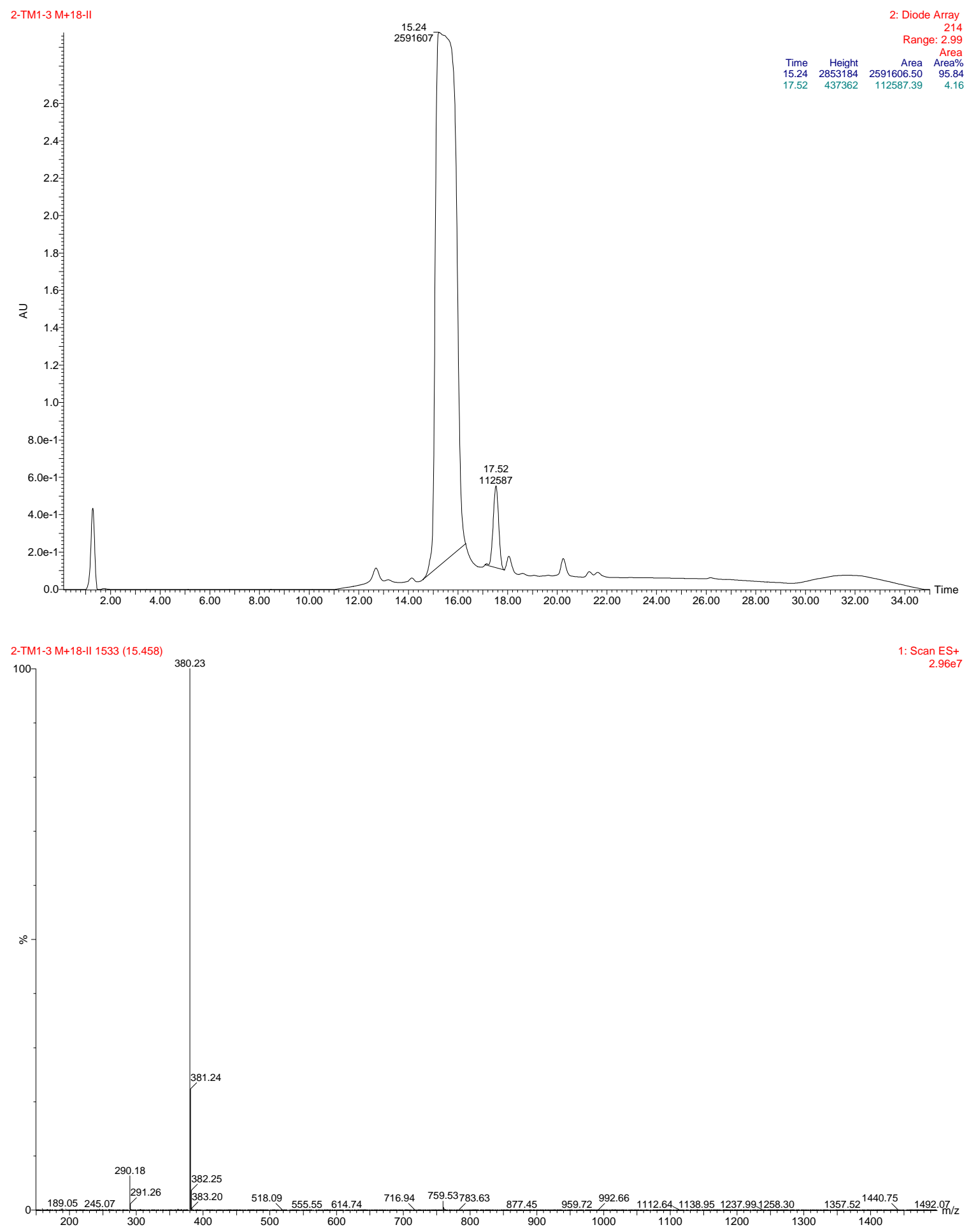


(5) Hemiaminal ${ }^{1} \mathbf{H}$-NMR from $4 \mathbf{a}+N$-Bn-Gly-OEt.

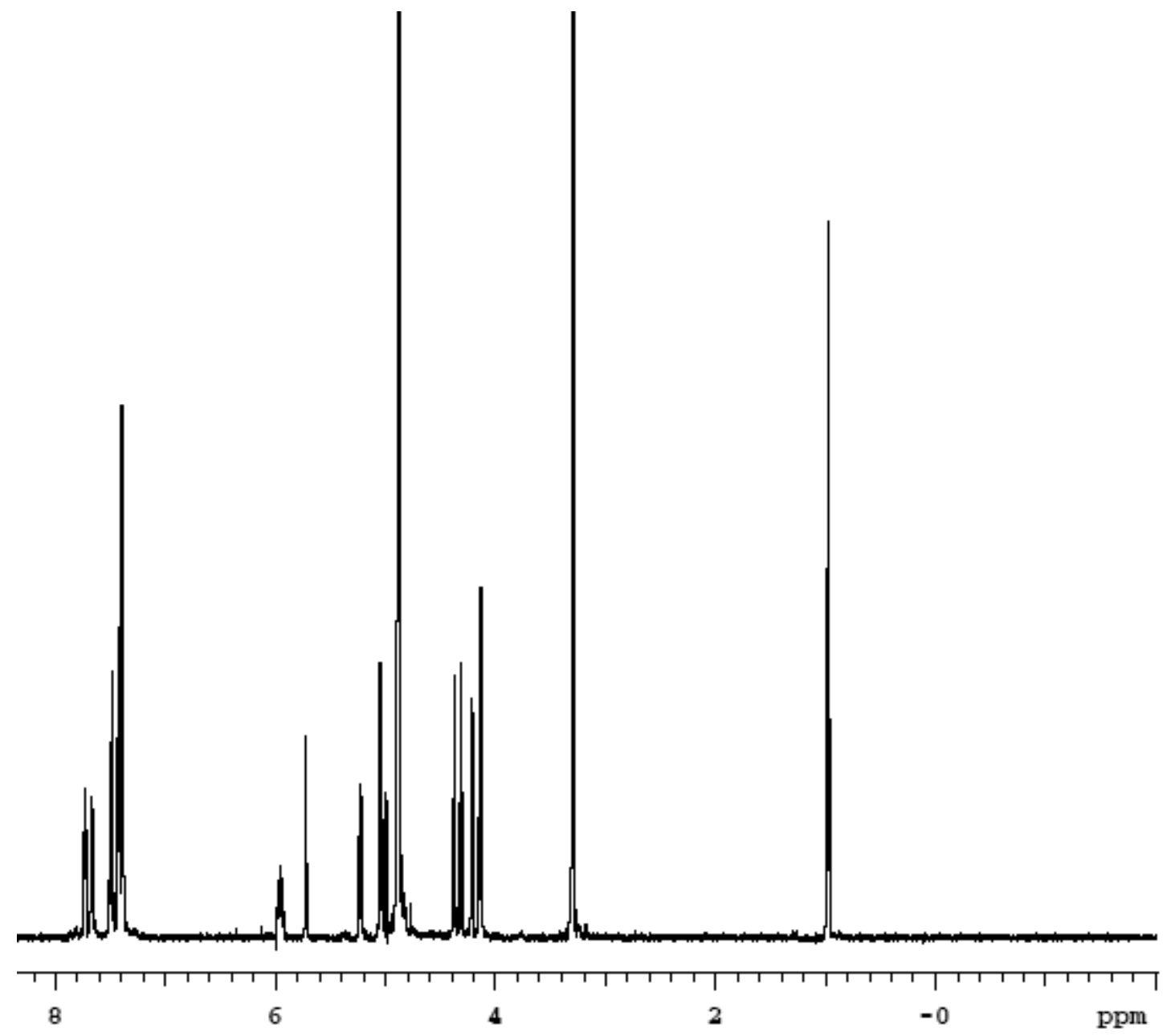


${ }^{1}{ }_{H}$ NMR, ${ }^{13}$ C NMR, HPLC/MS Spectra of Intermediates and Selected Library Members<smiles>CCOC(OCC)c1nc2ccccc2[nH]1</smiles>

$2 a$

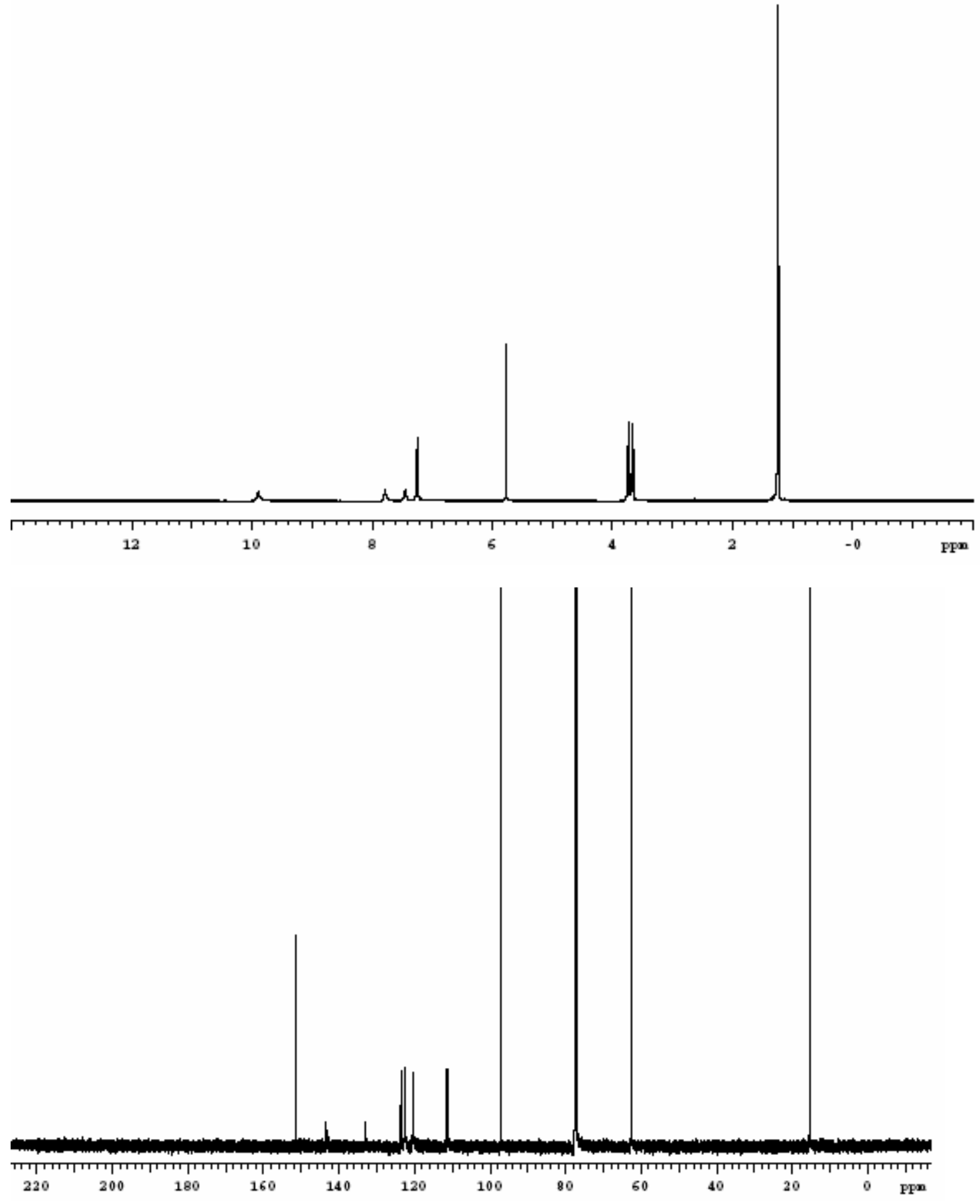




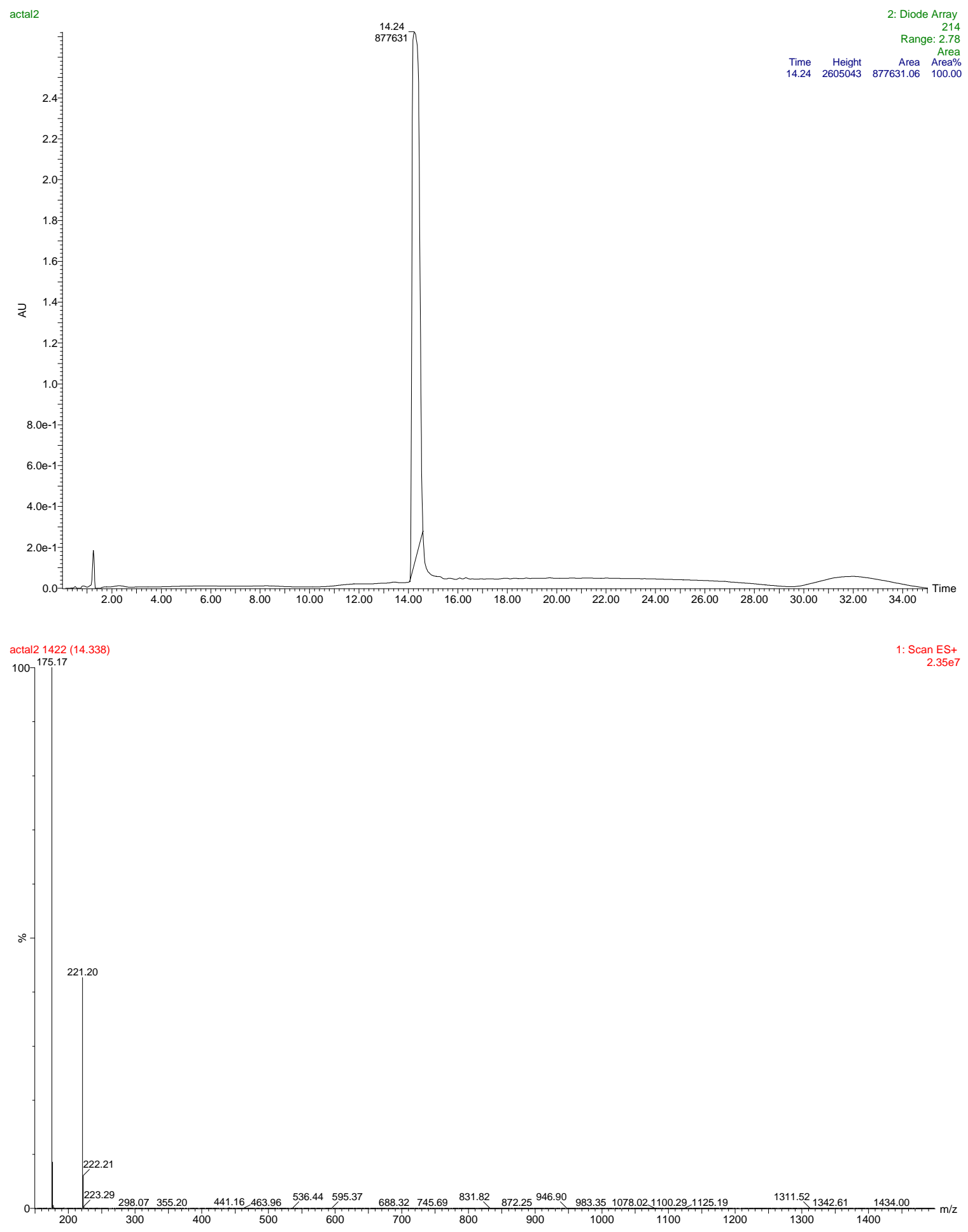




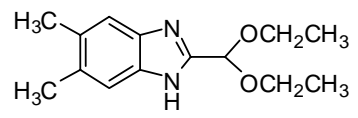

2b
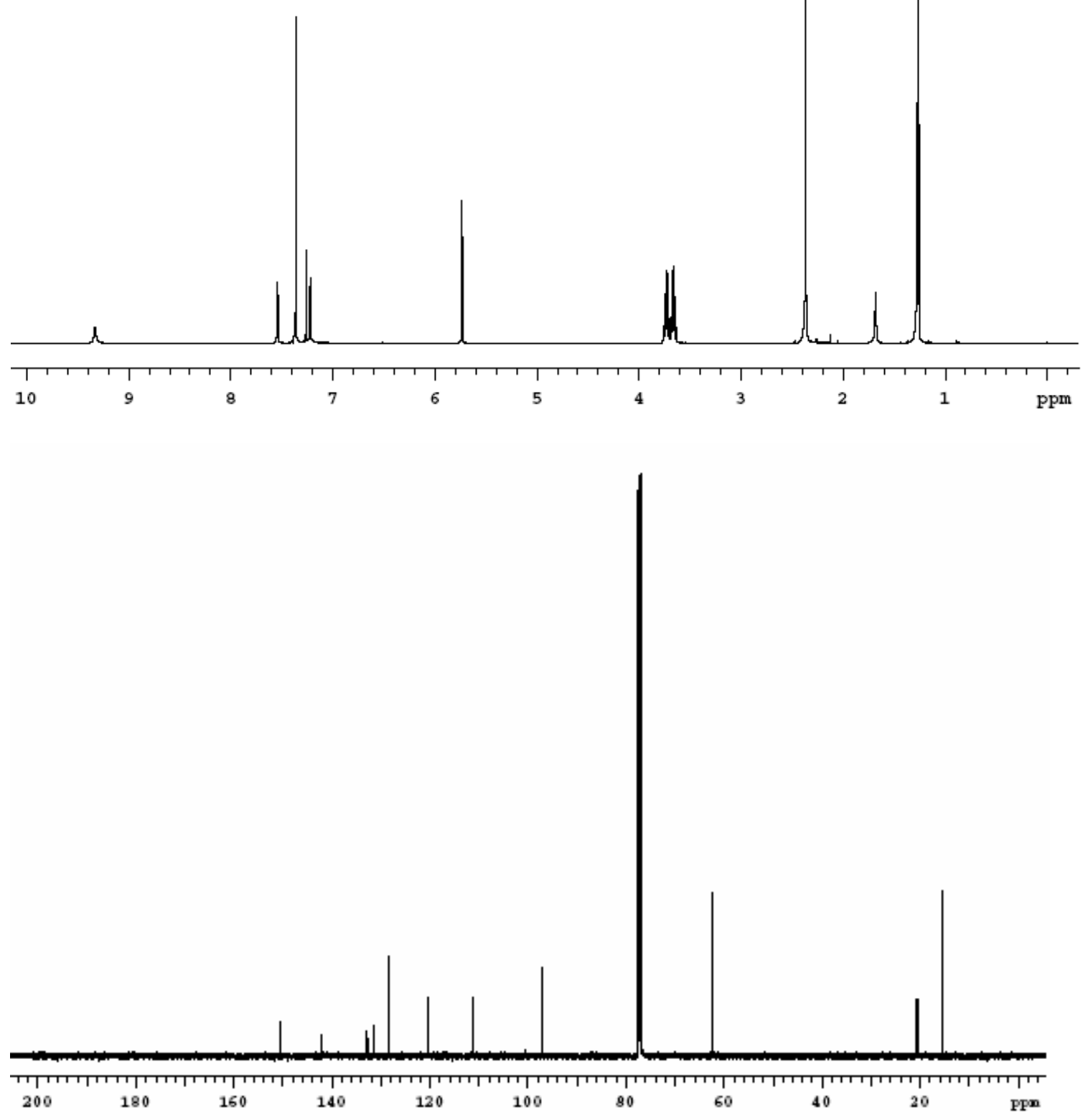

S21 
$2 b$

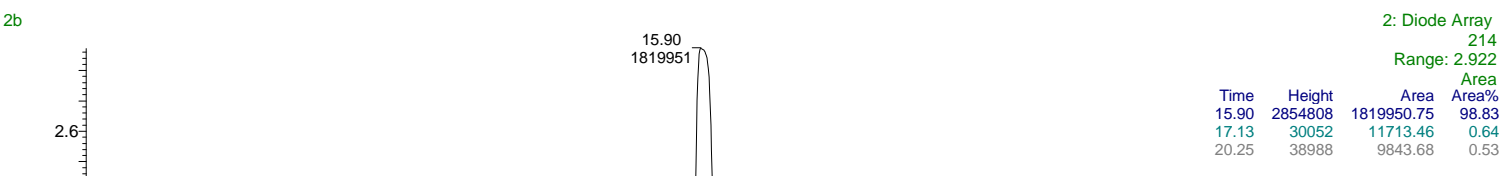

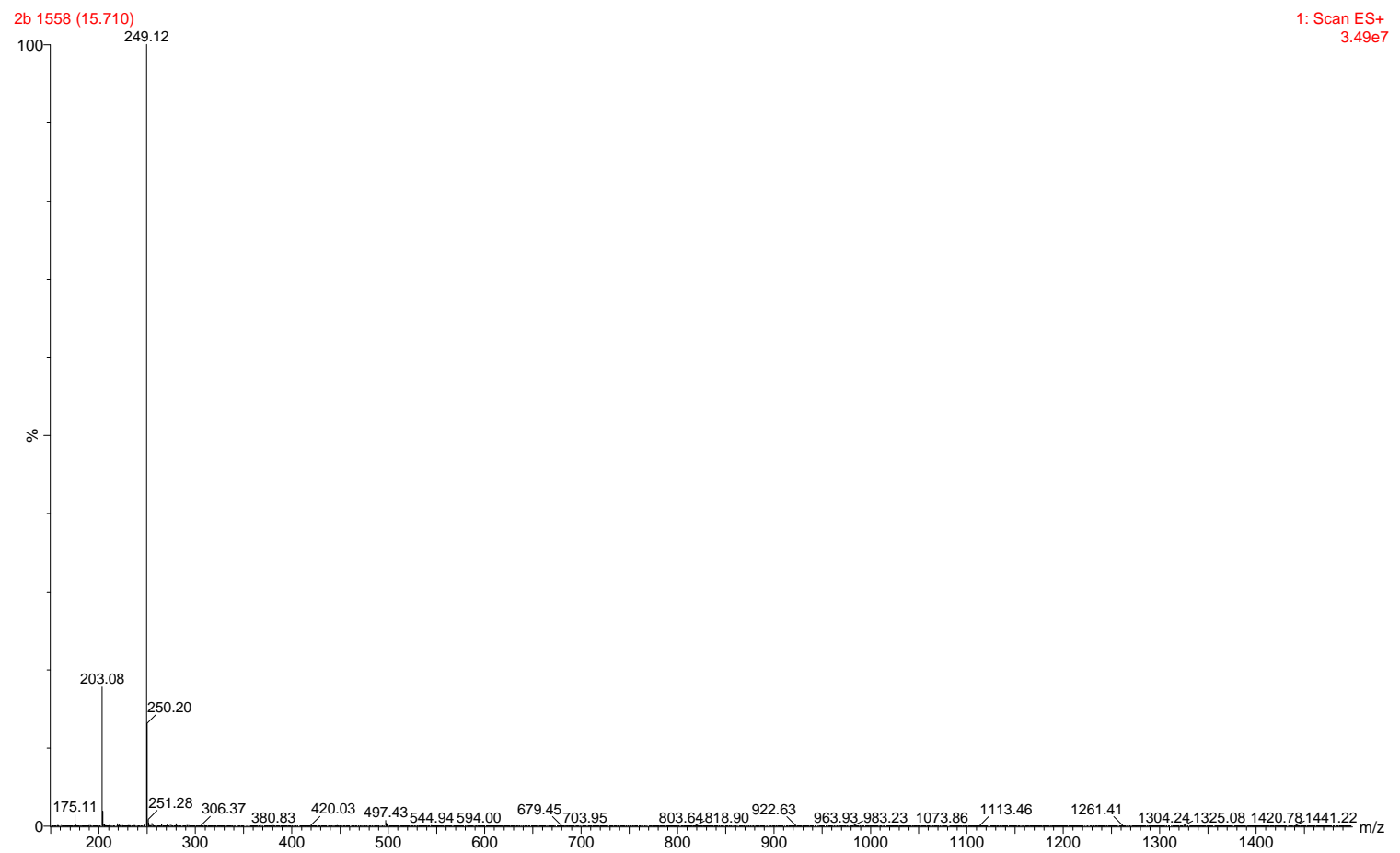



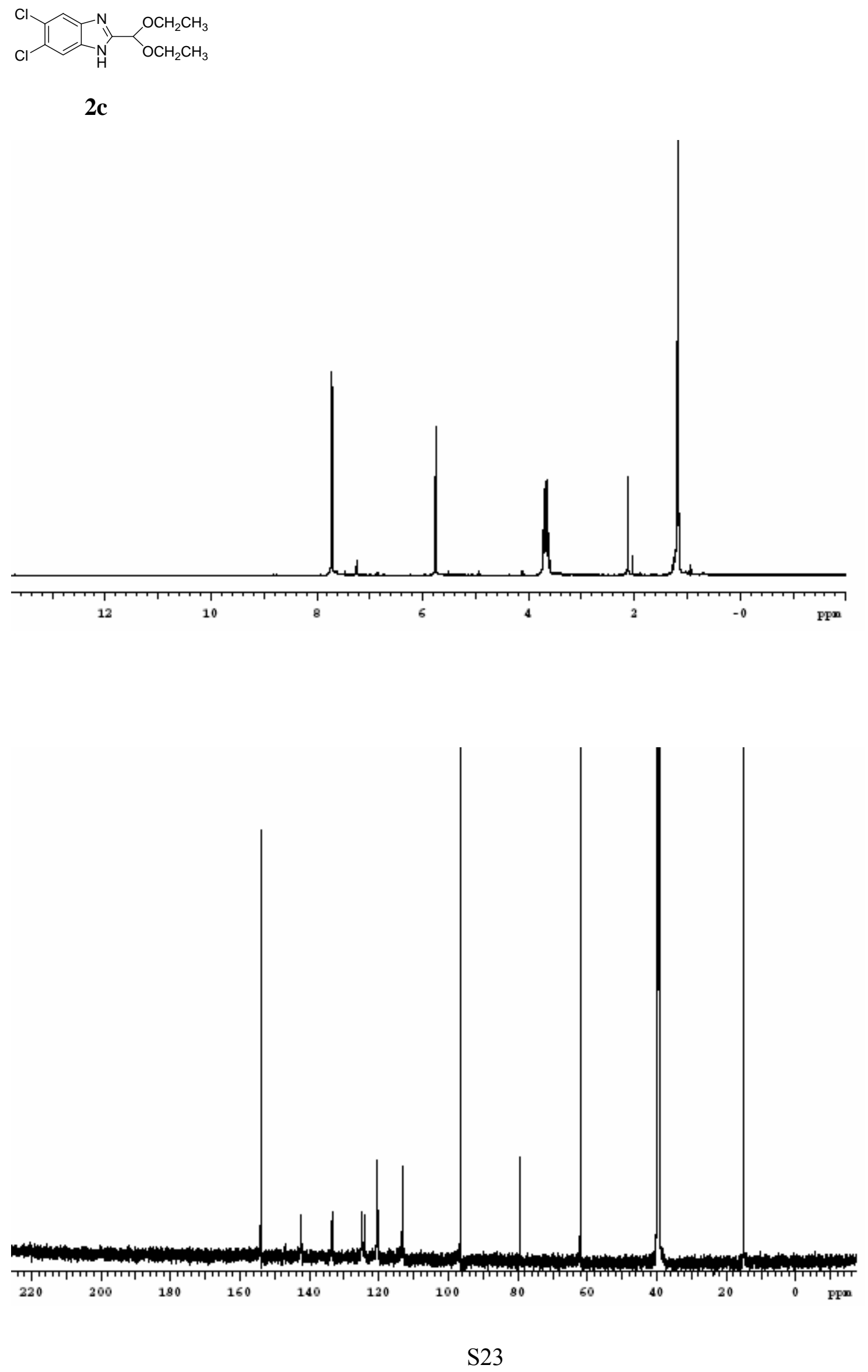


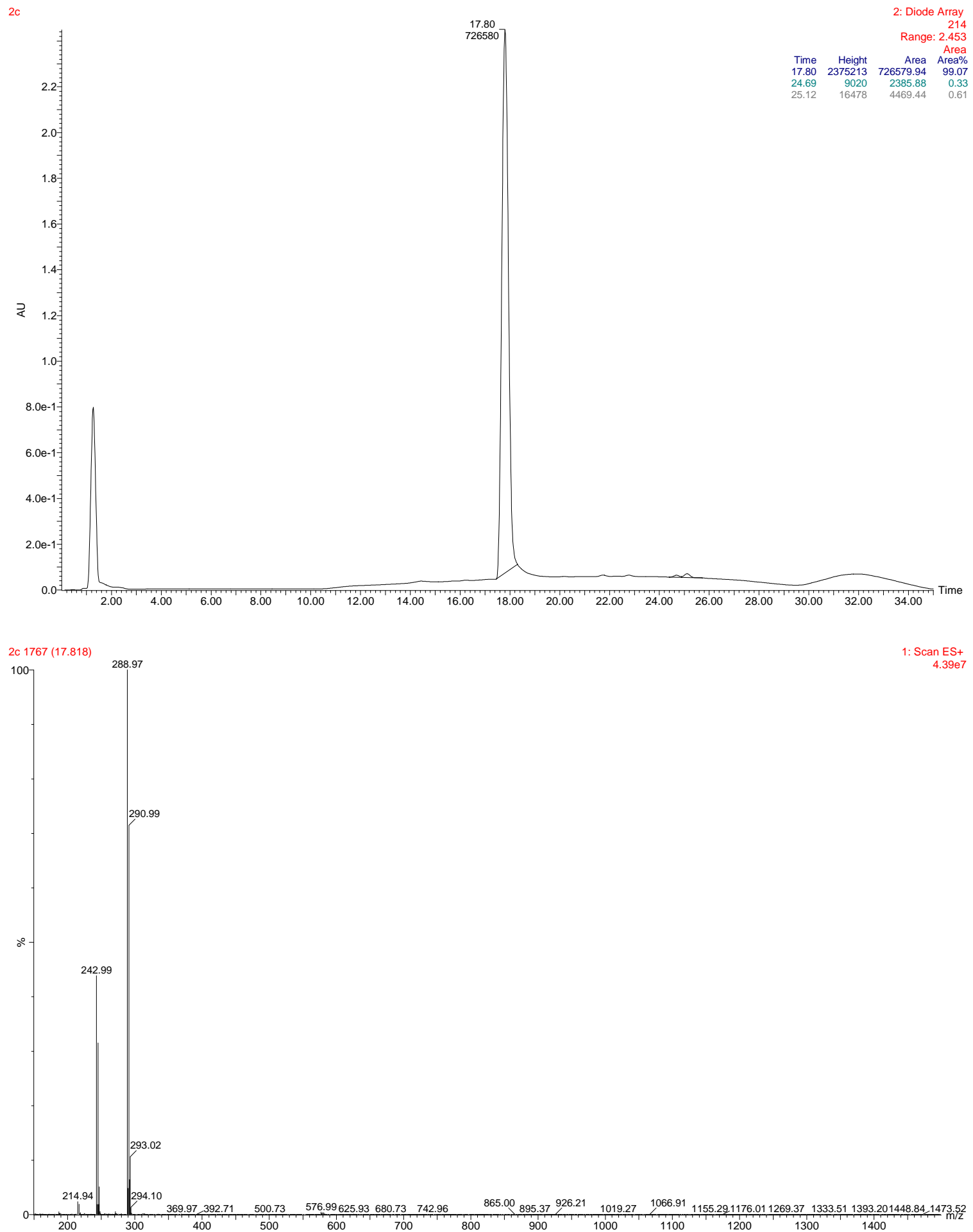




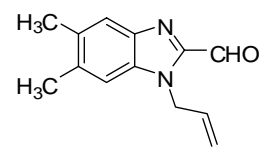

4b
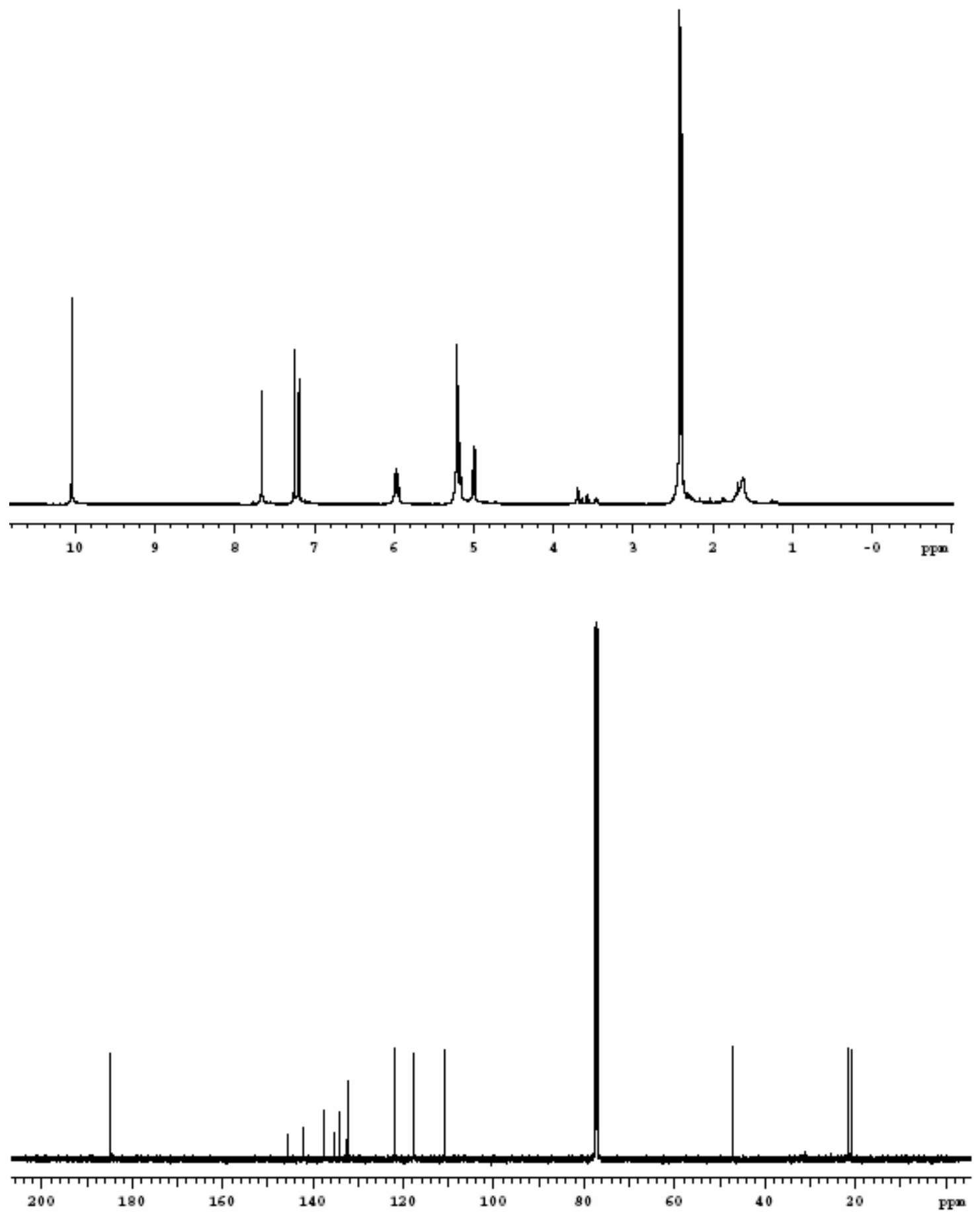

S25 
$4 b$

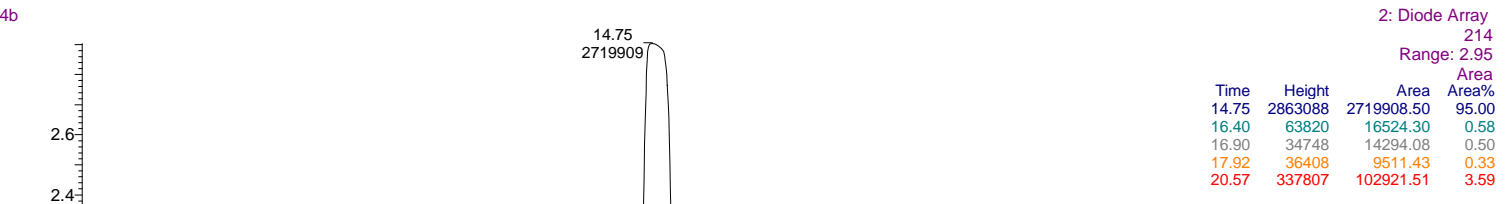

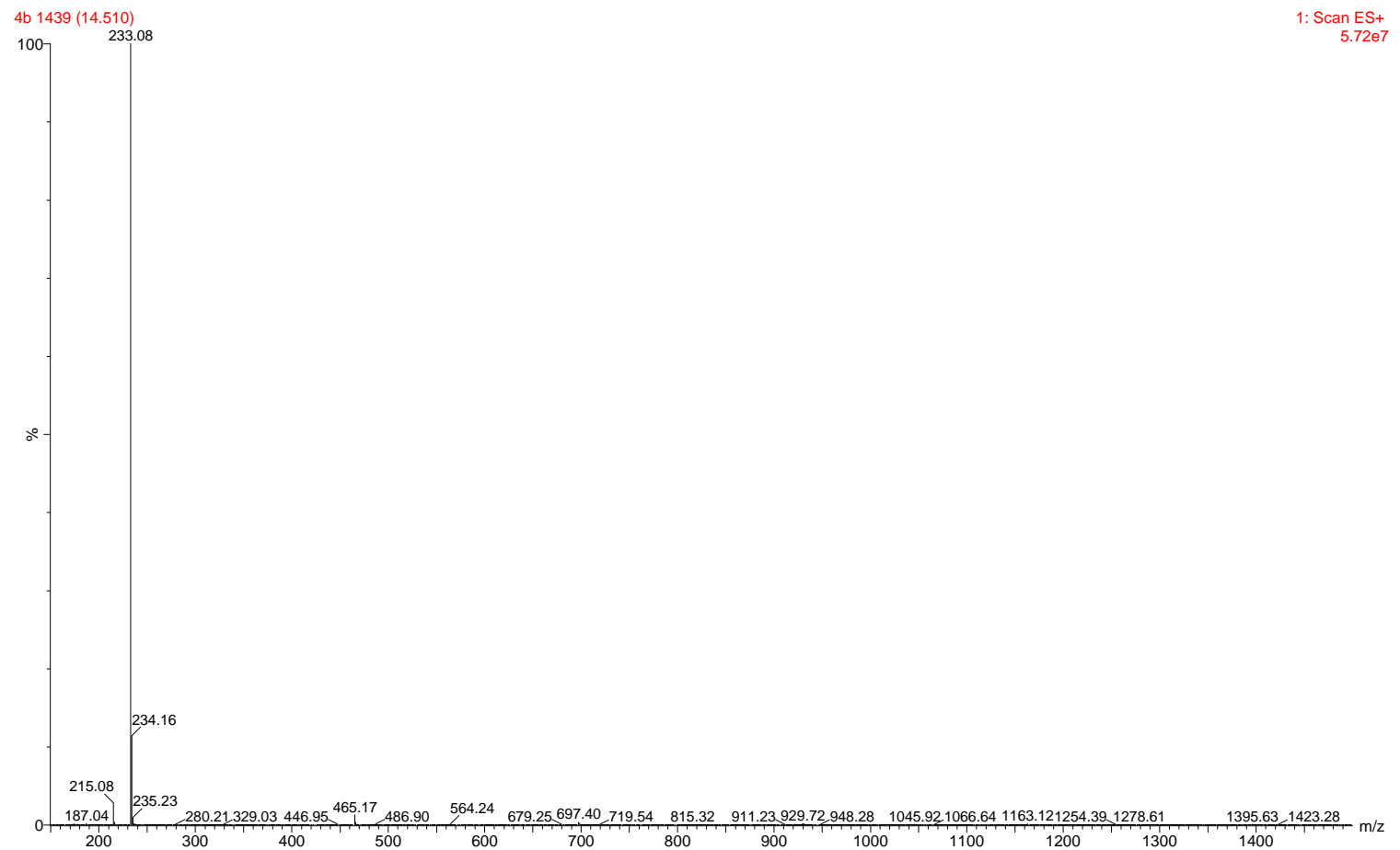




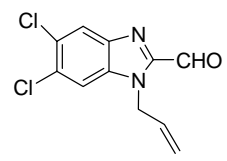

4c
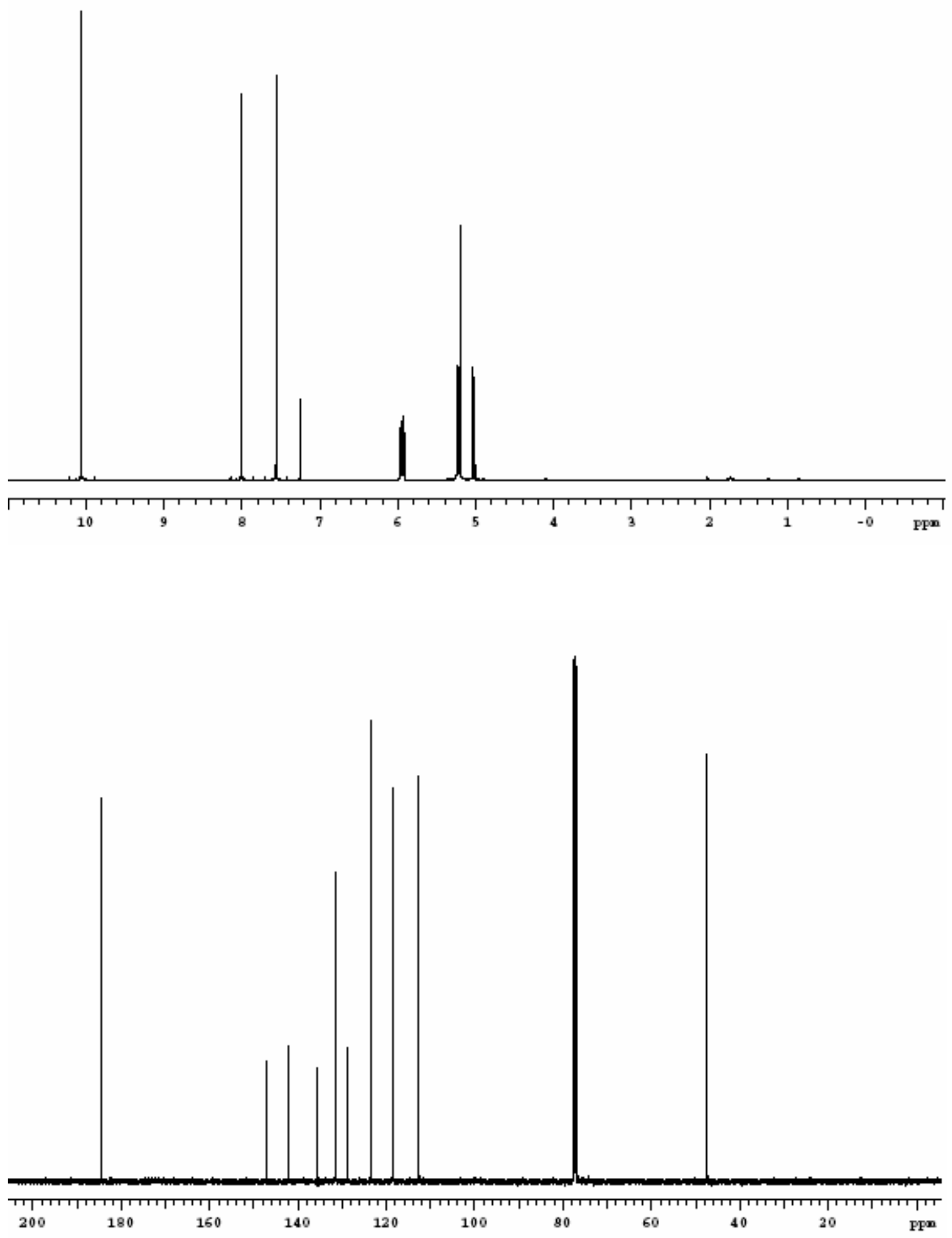

S27 


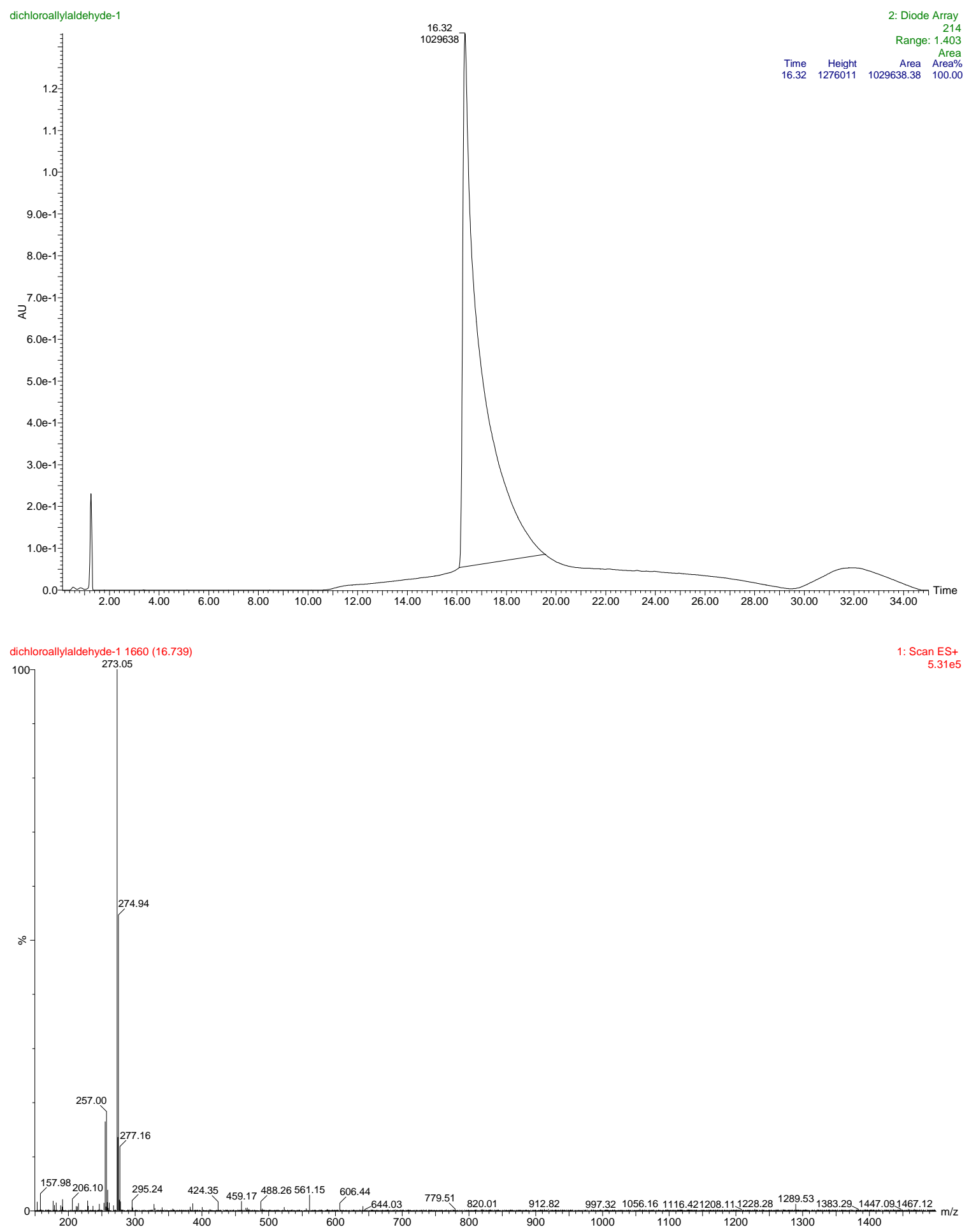




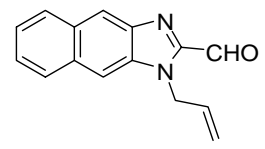

4d
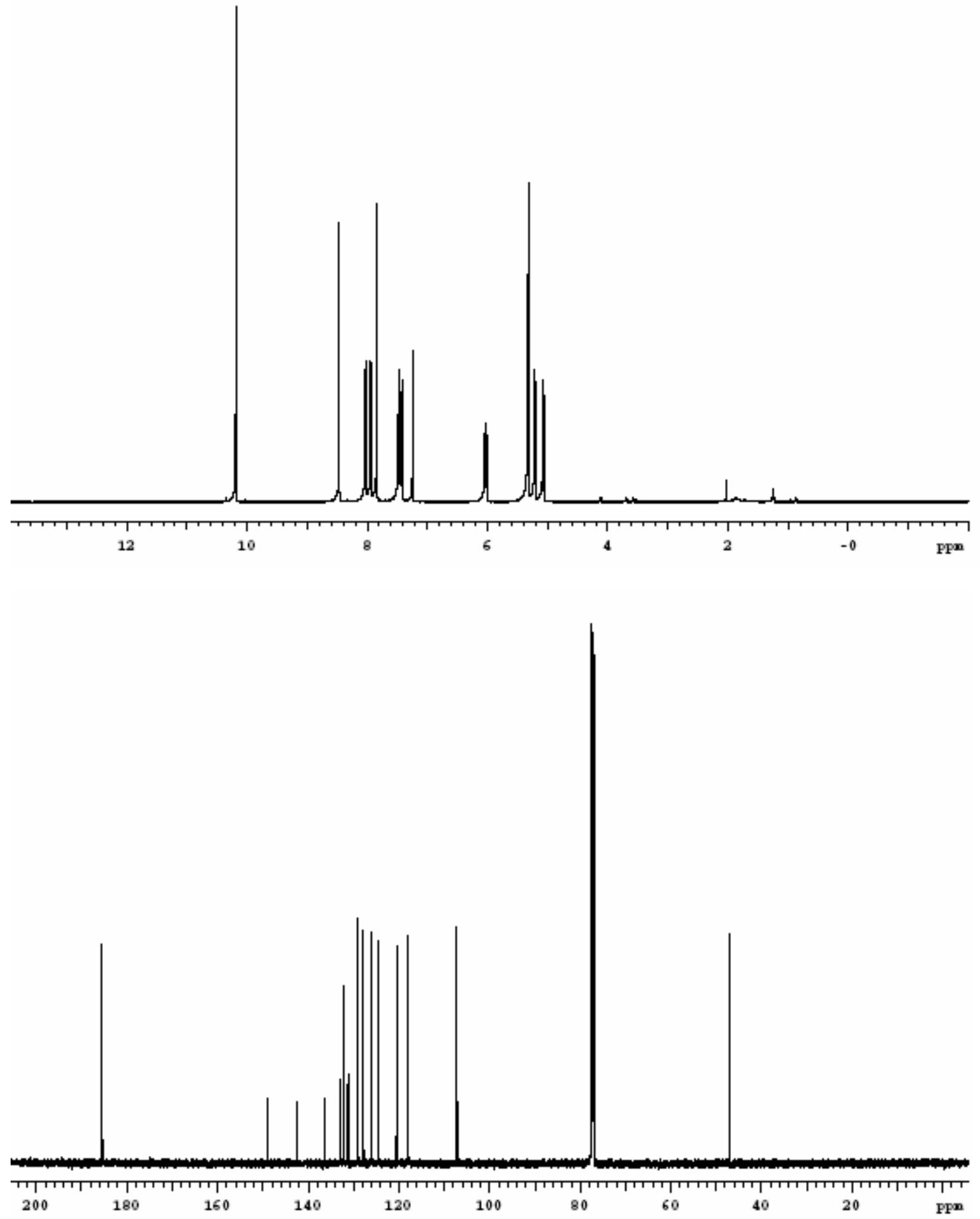
$4 d$
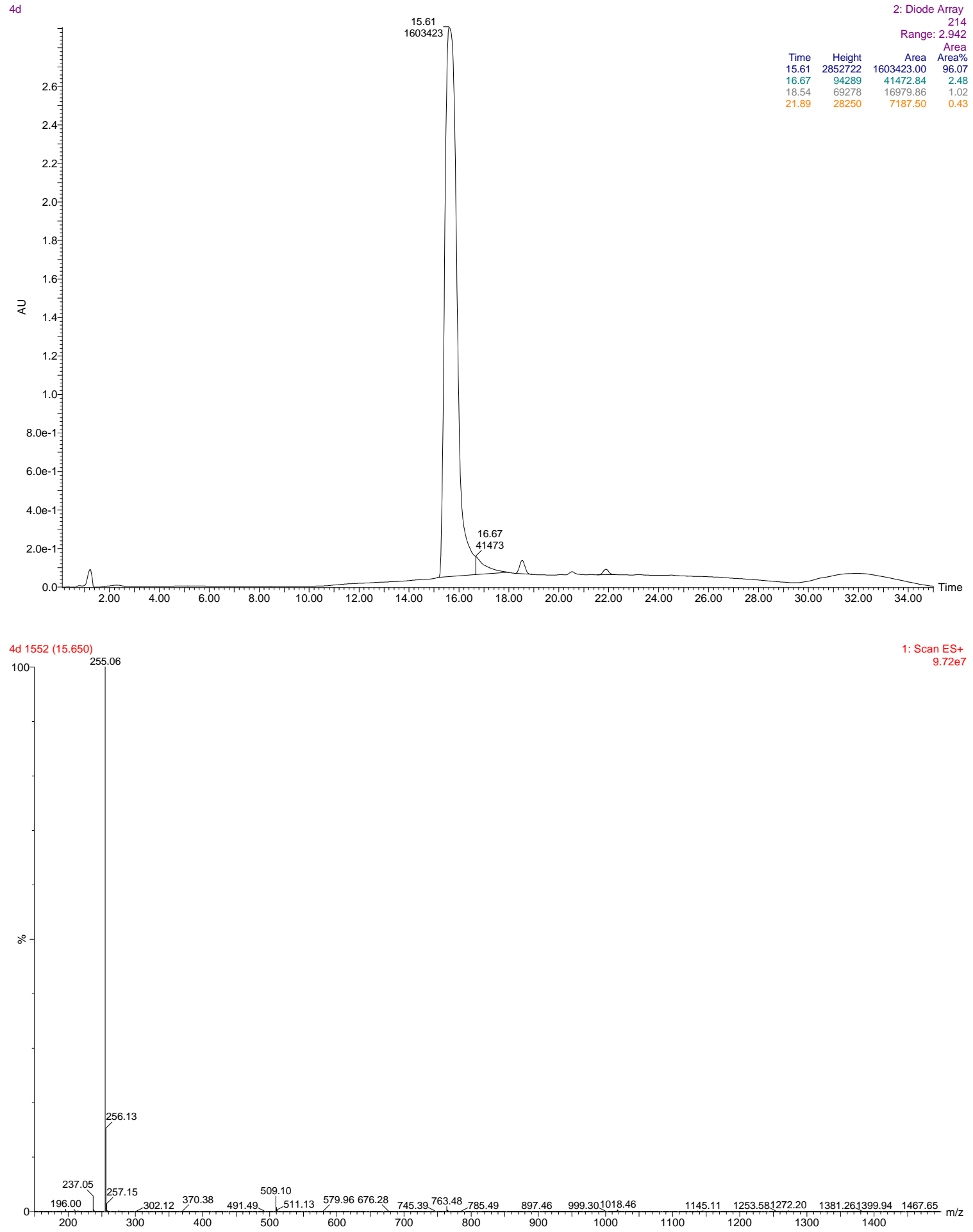

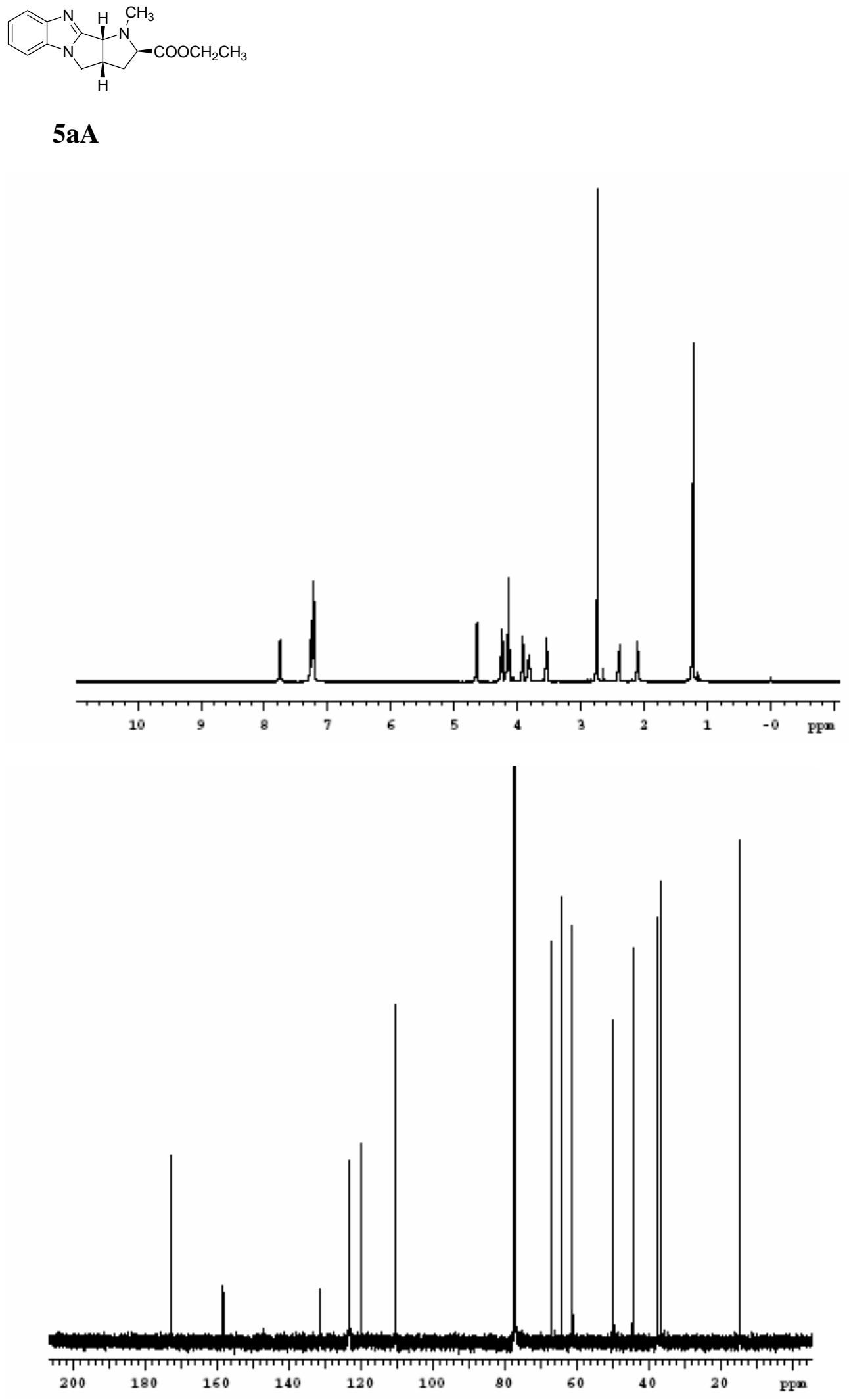

S31 

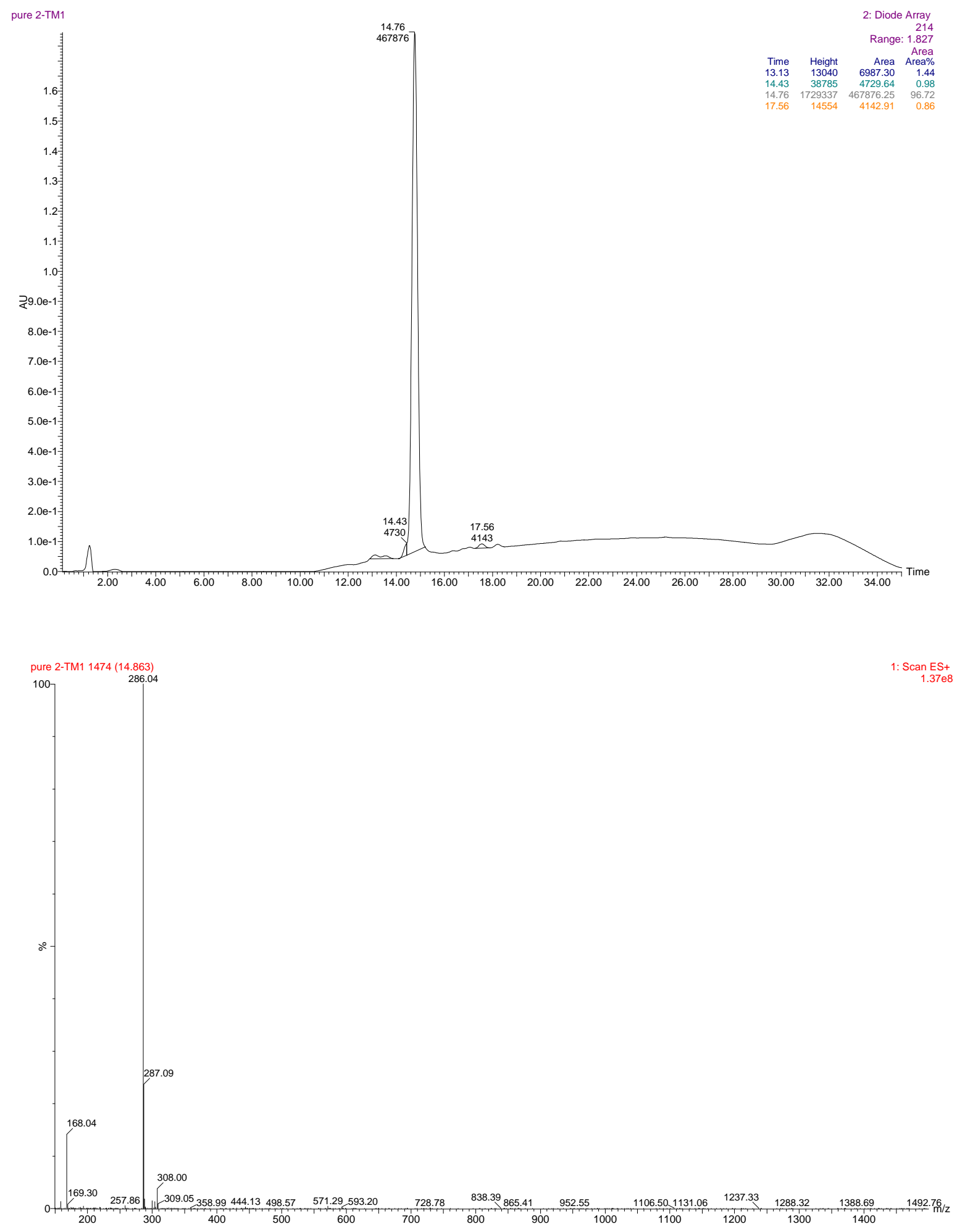


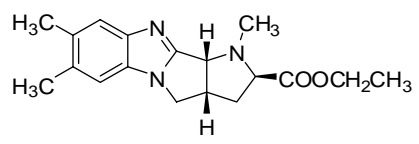

5bA
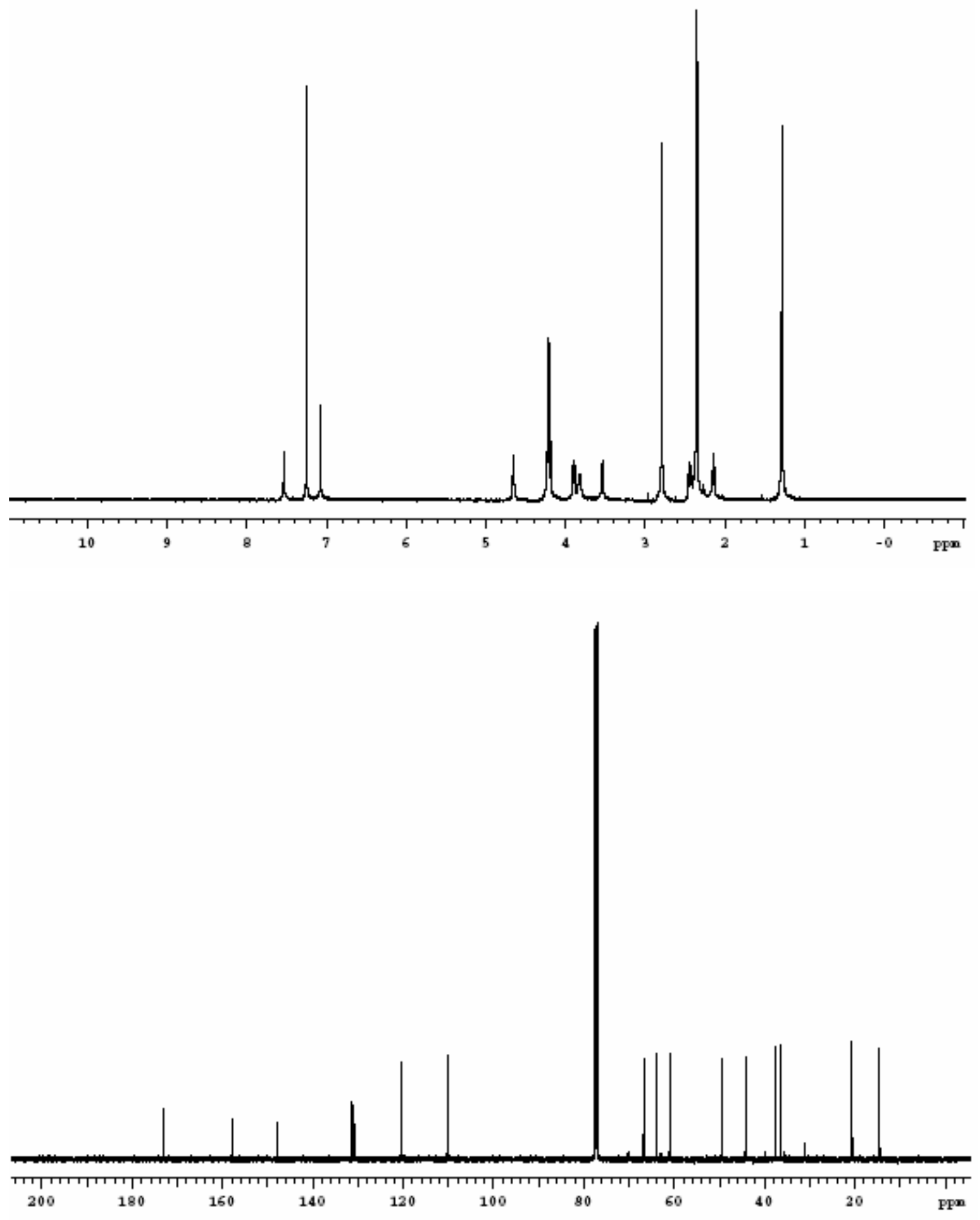

S33 


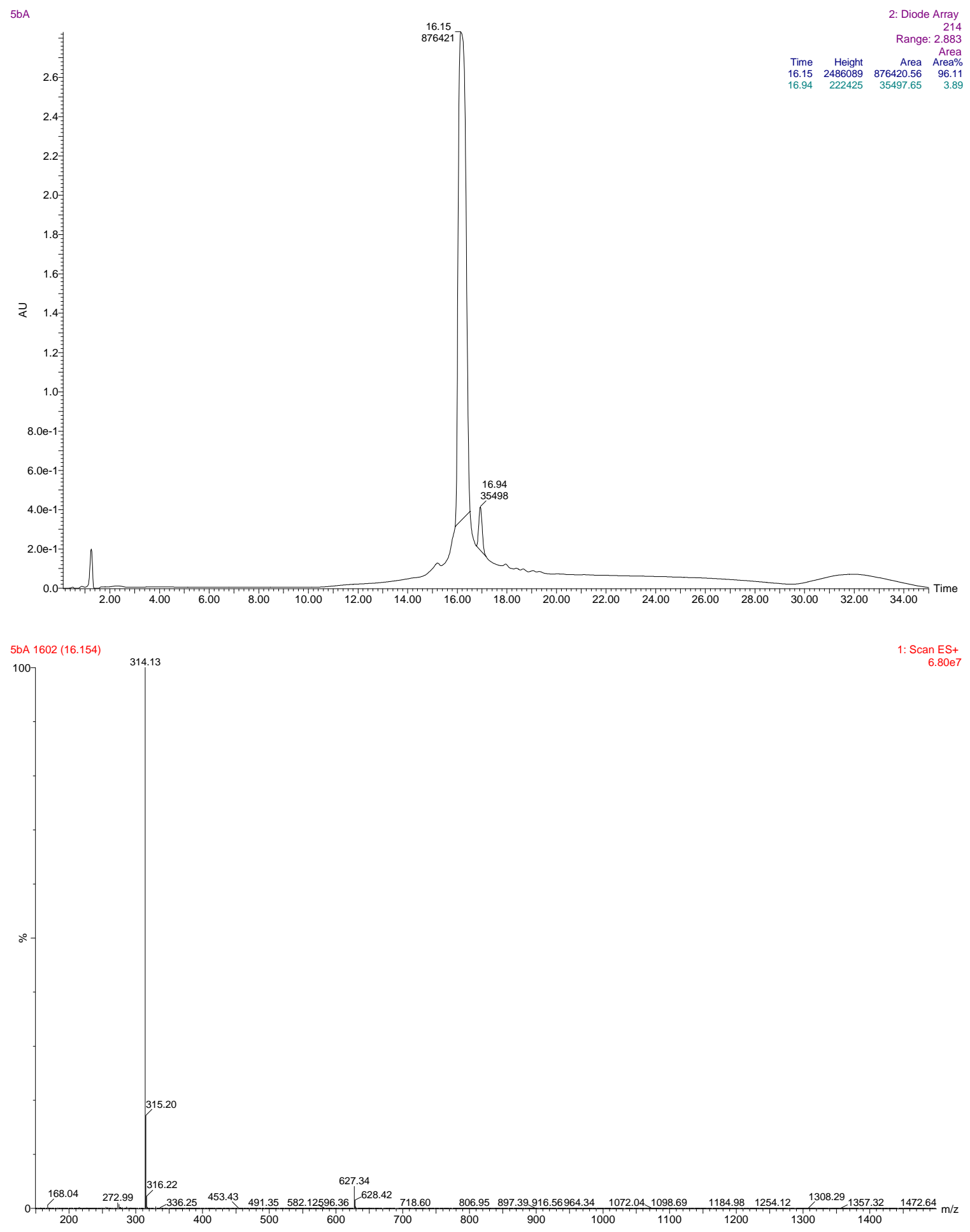




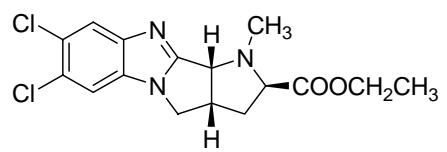

5cA
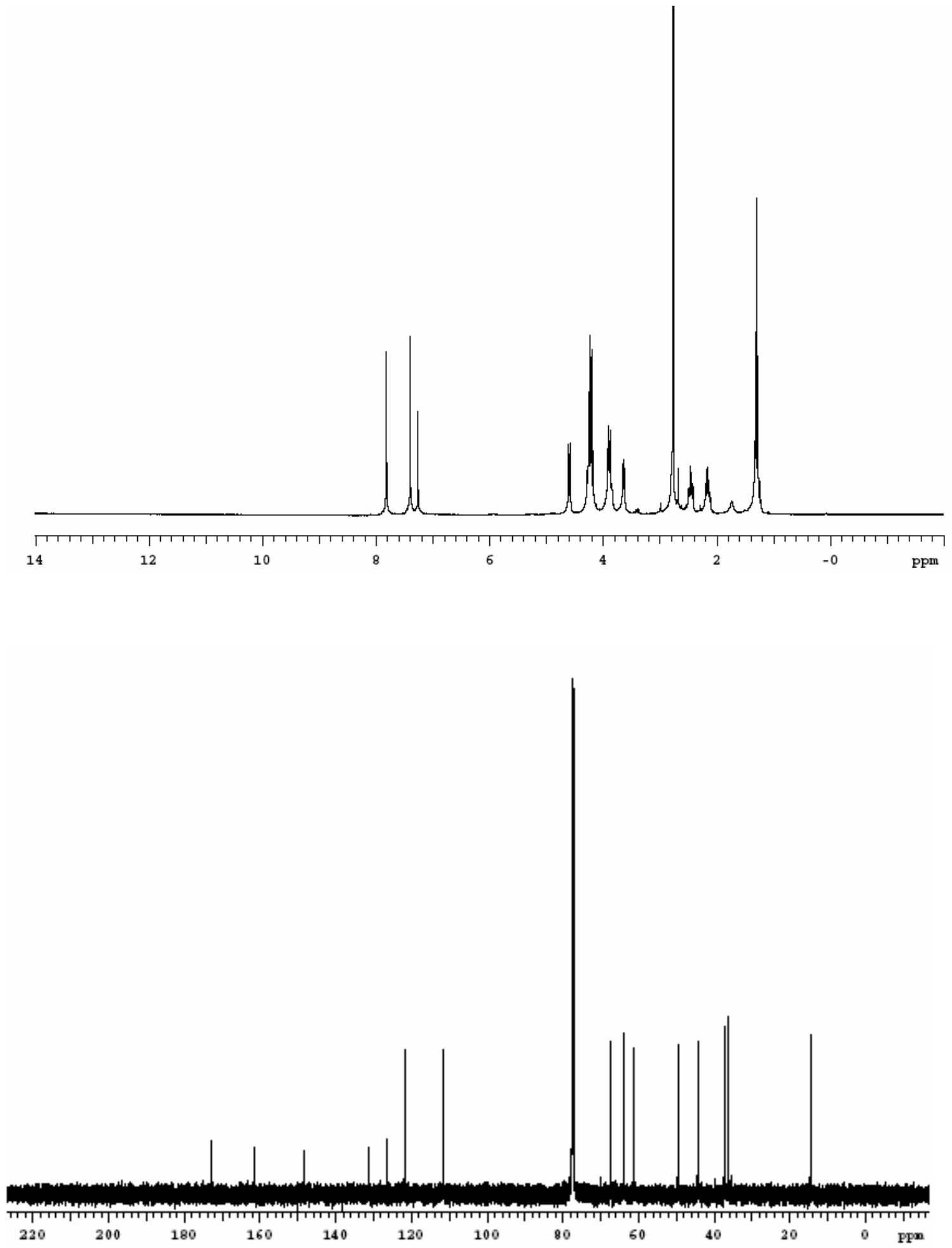

S35 


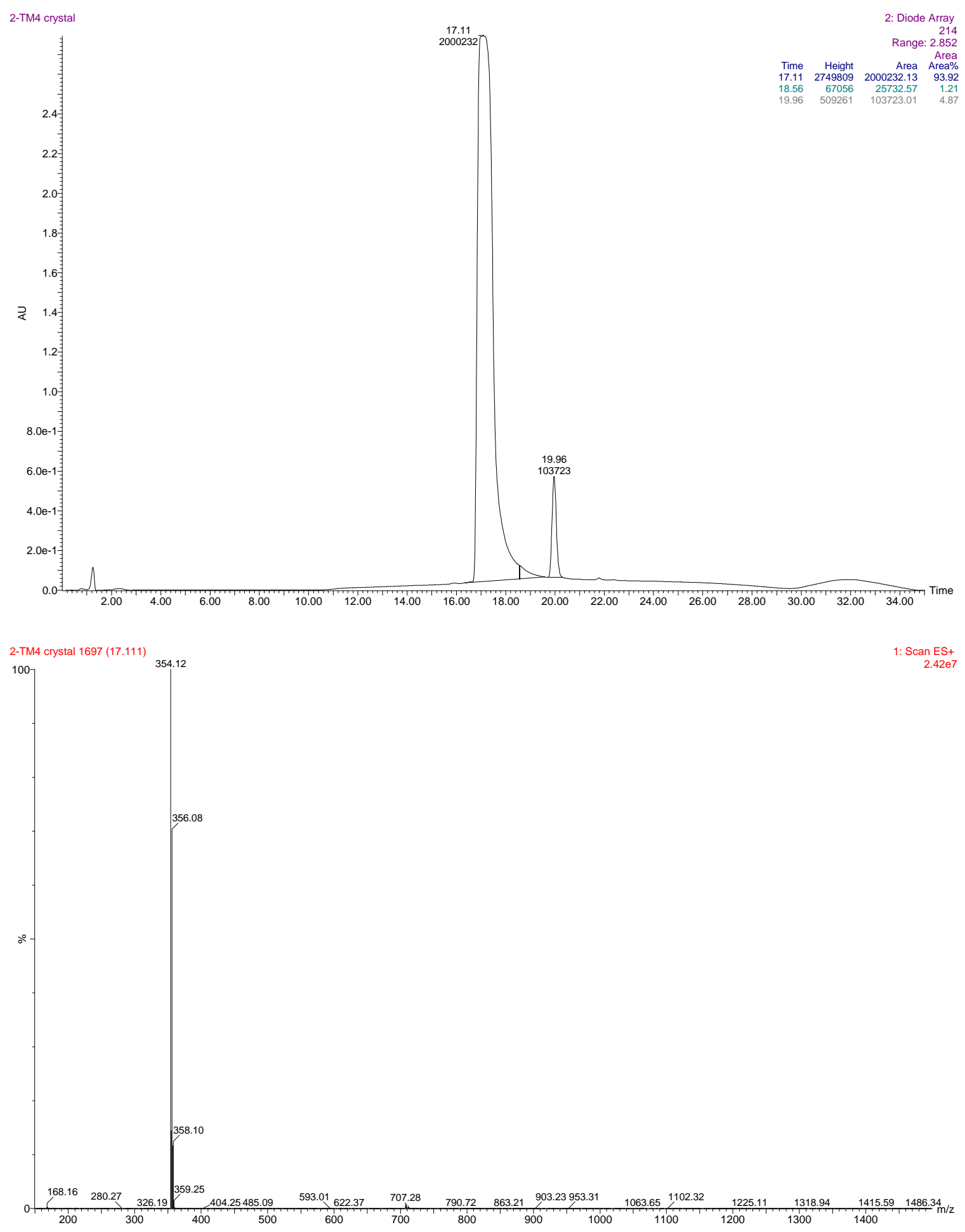



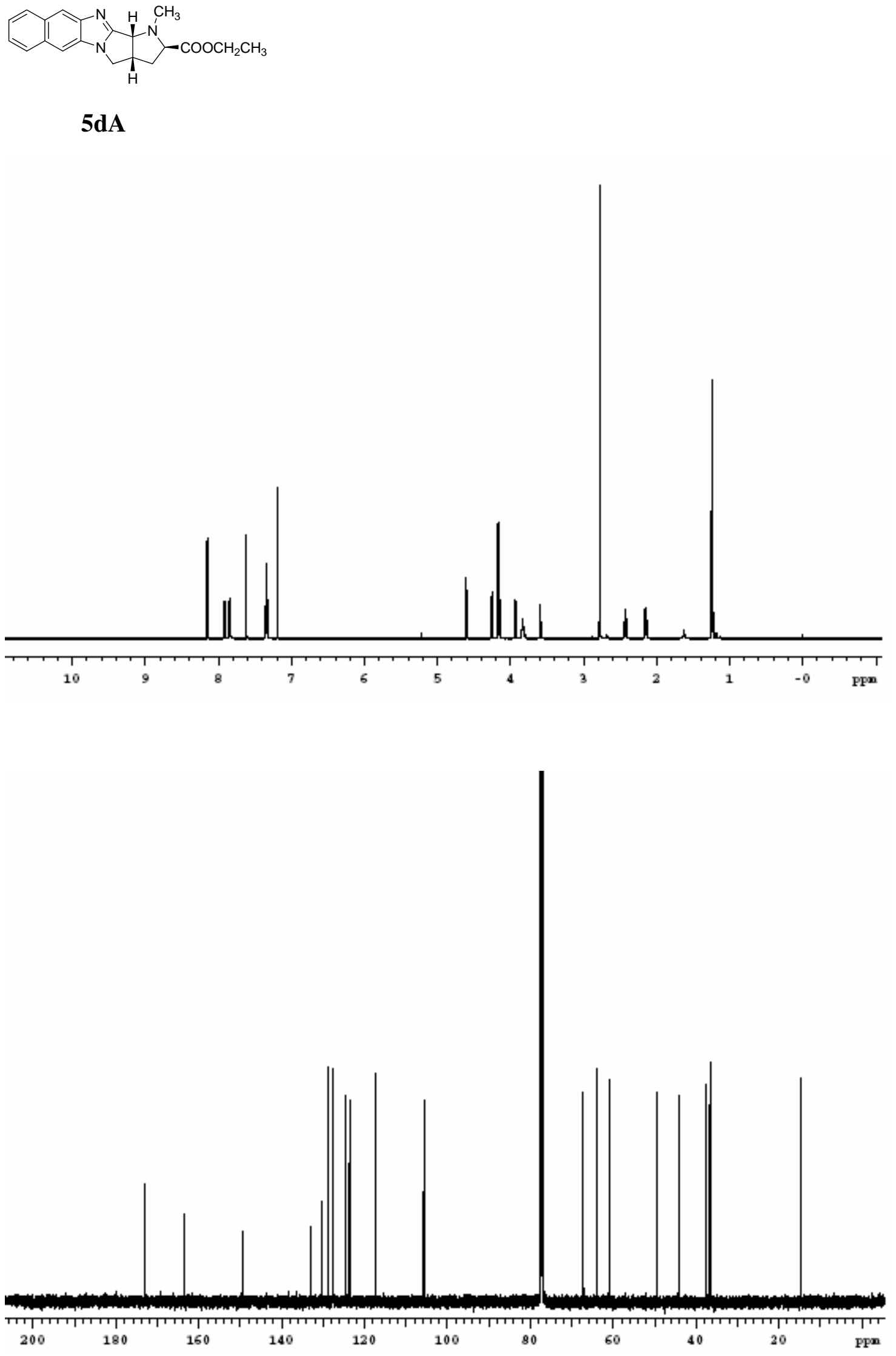

S37 


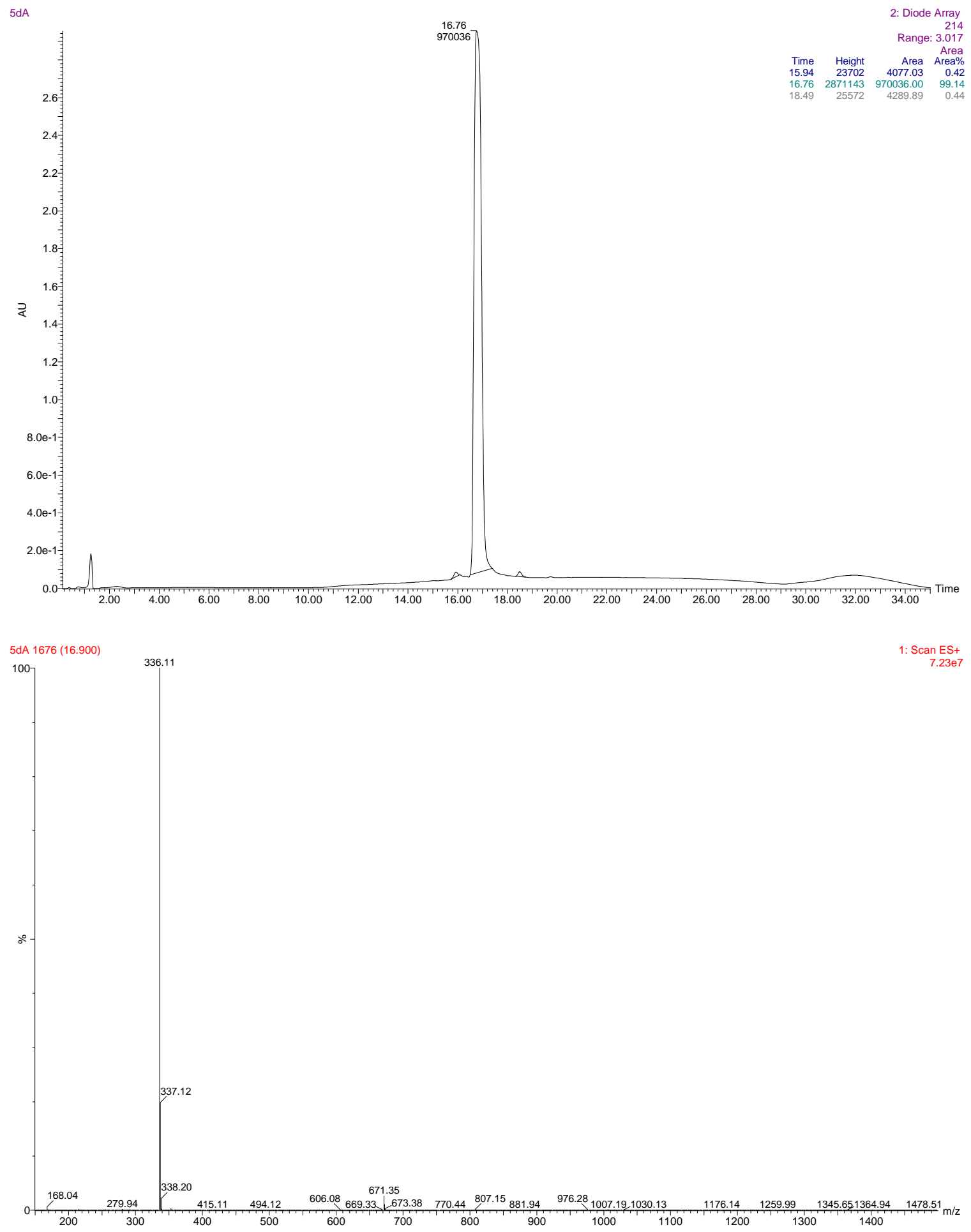




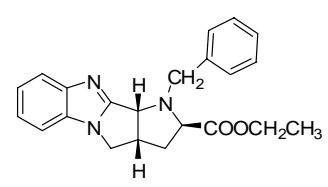

$5 a B$
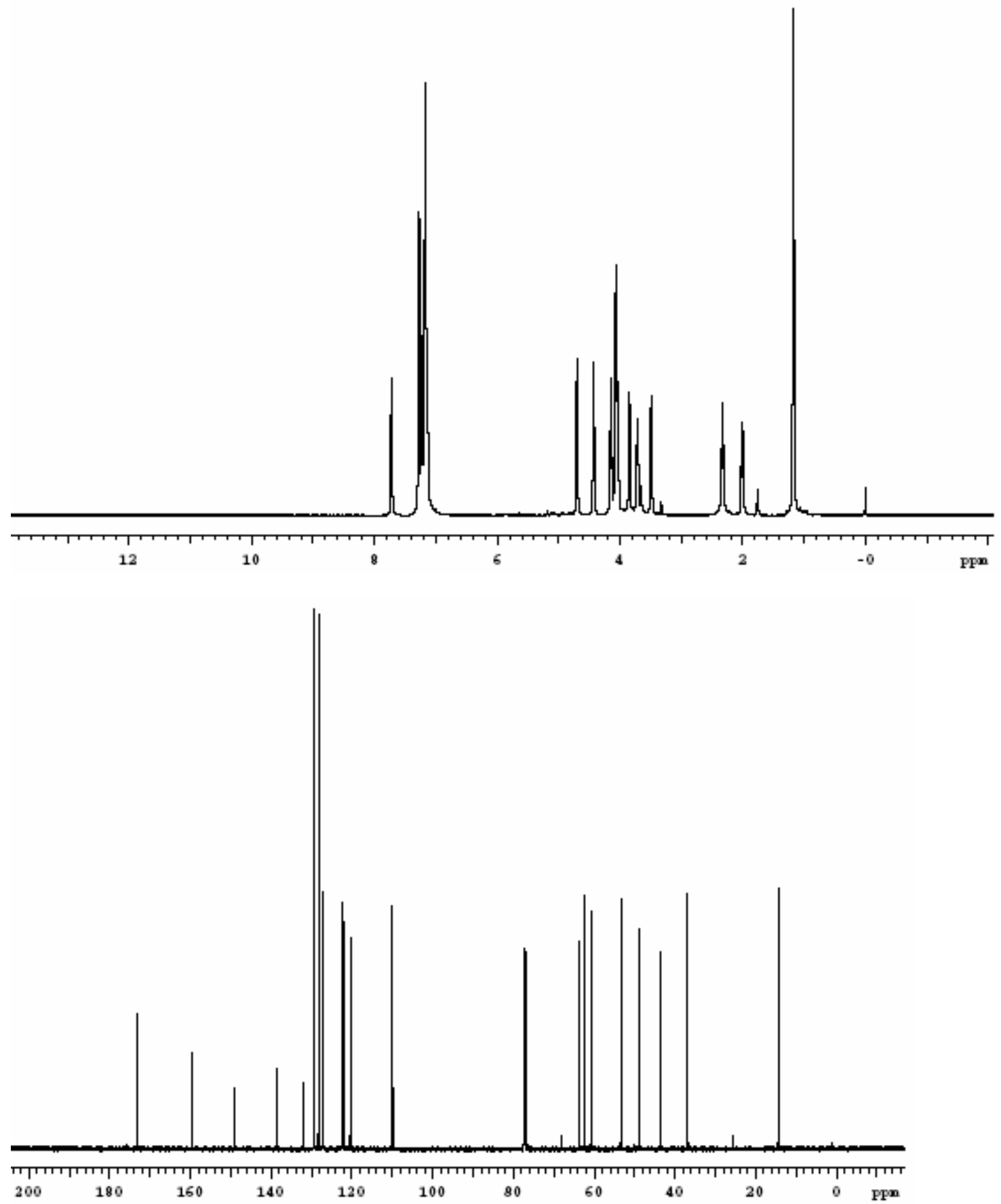


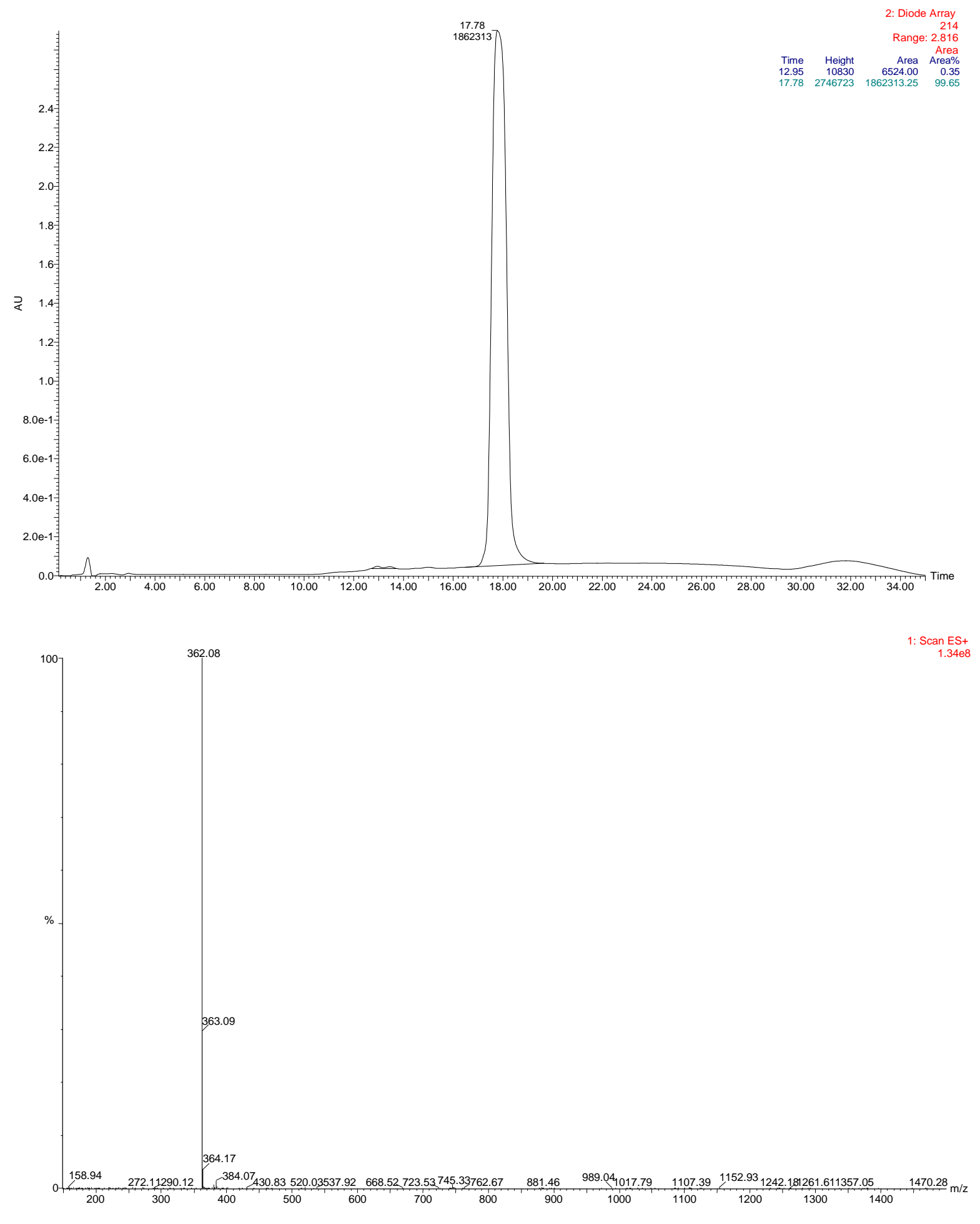




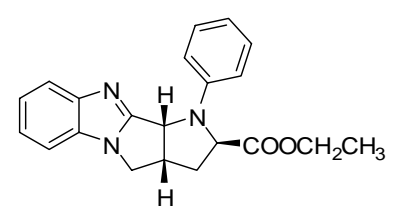

$5 a C$
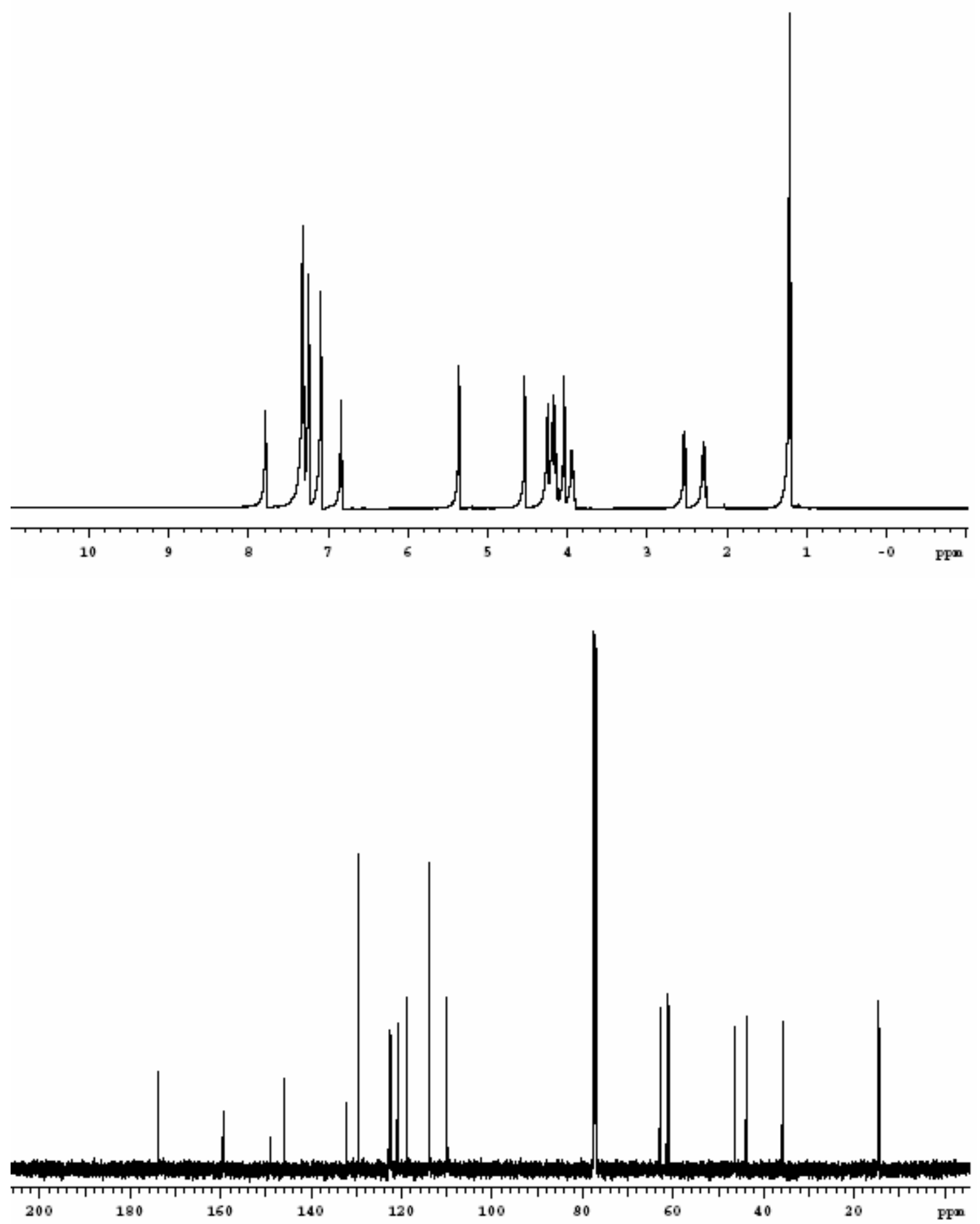


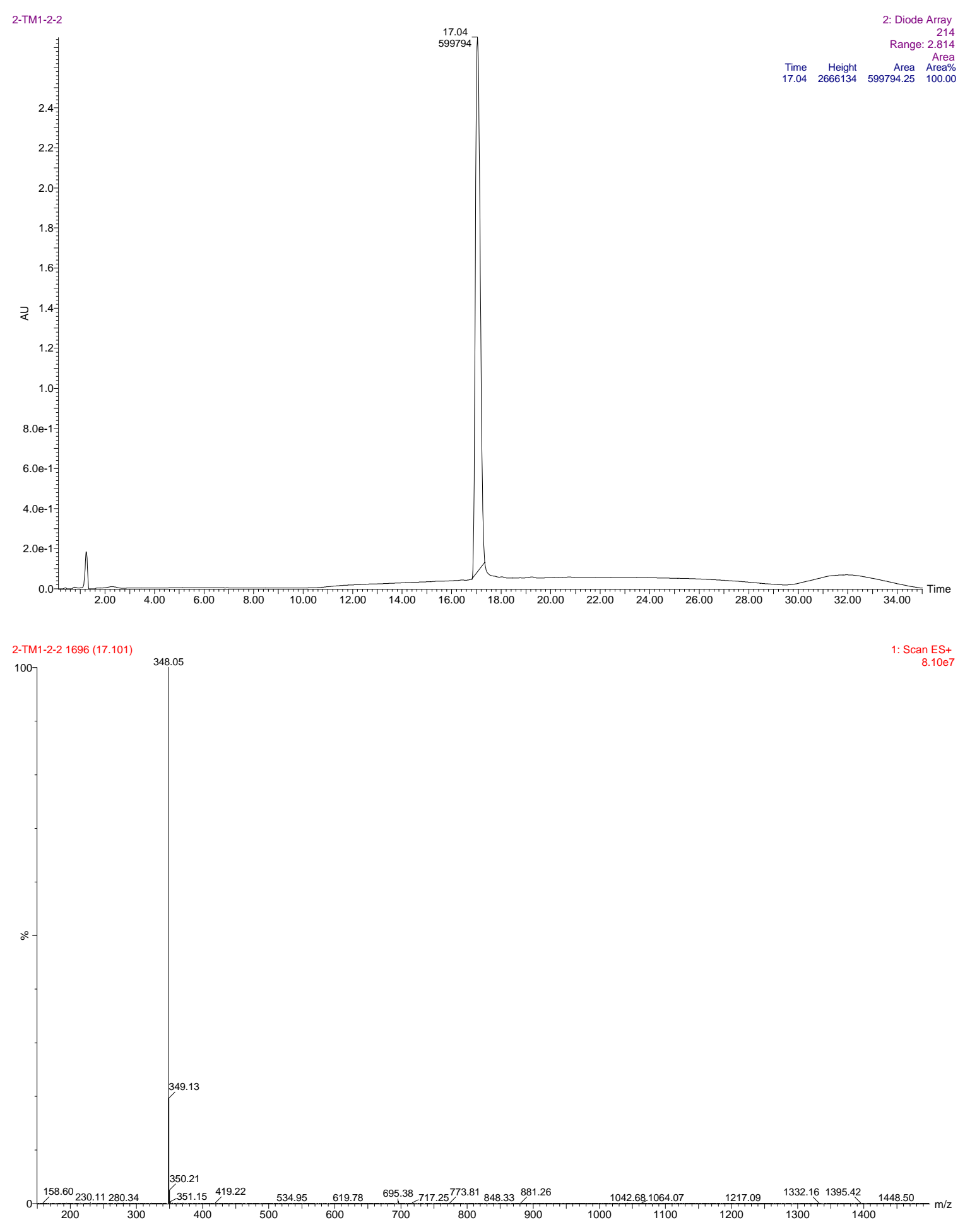




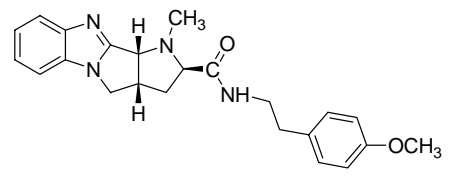

7aA $\{1\}$
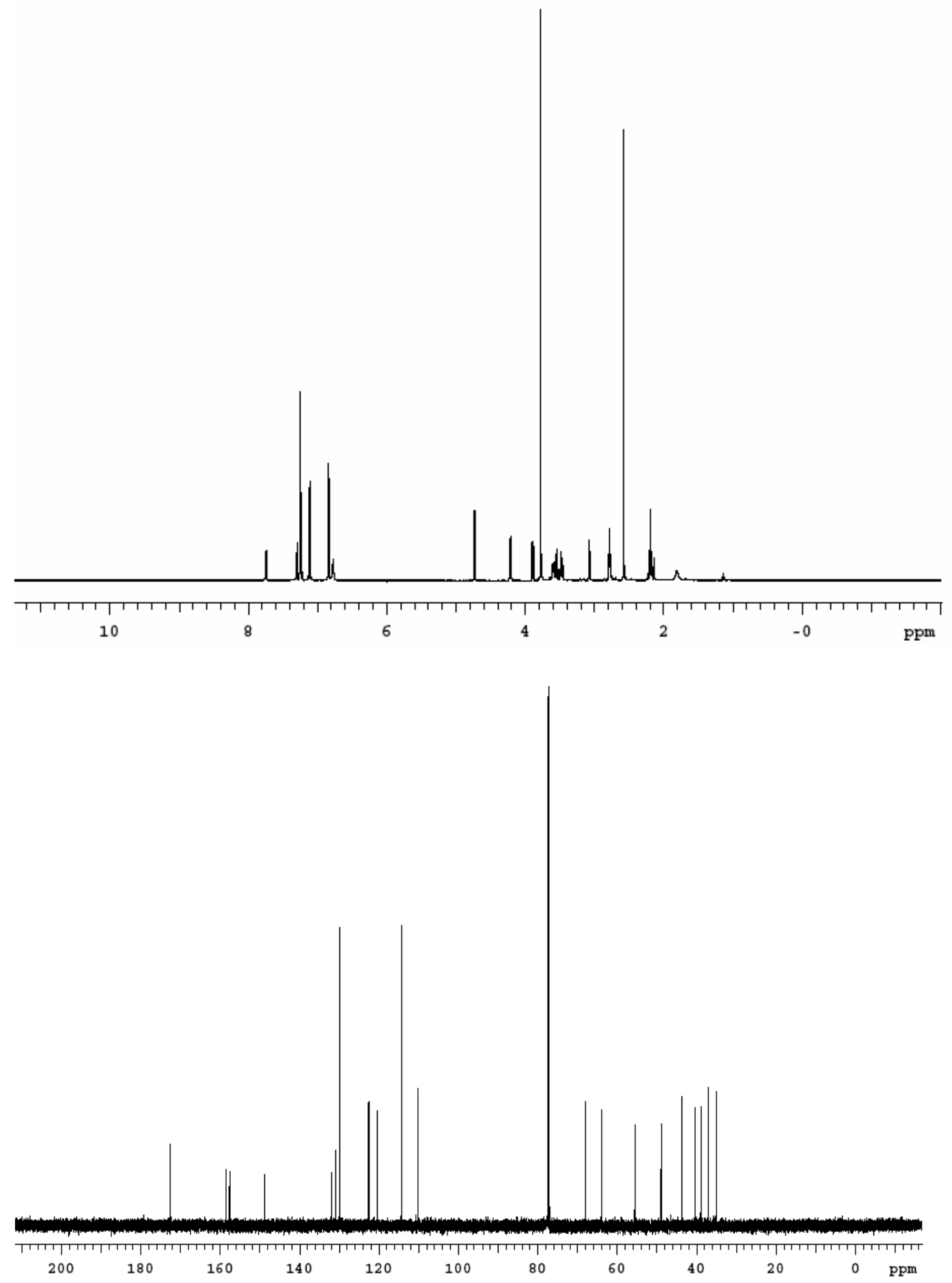


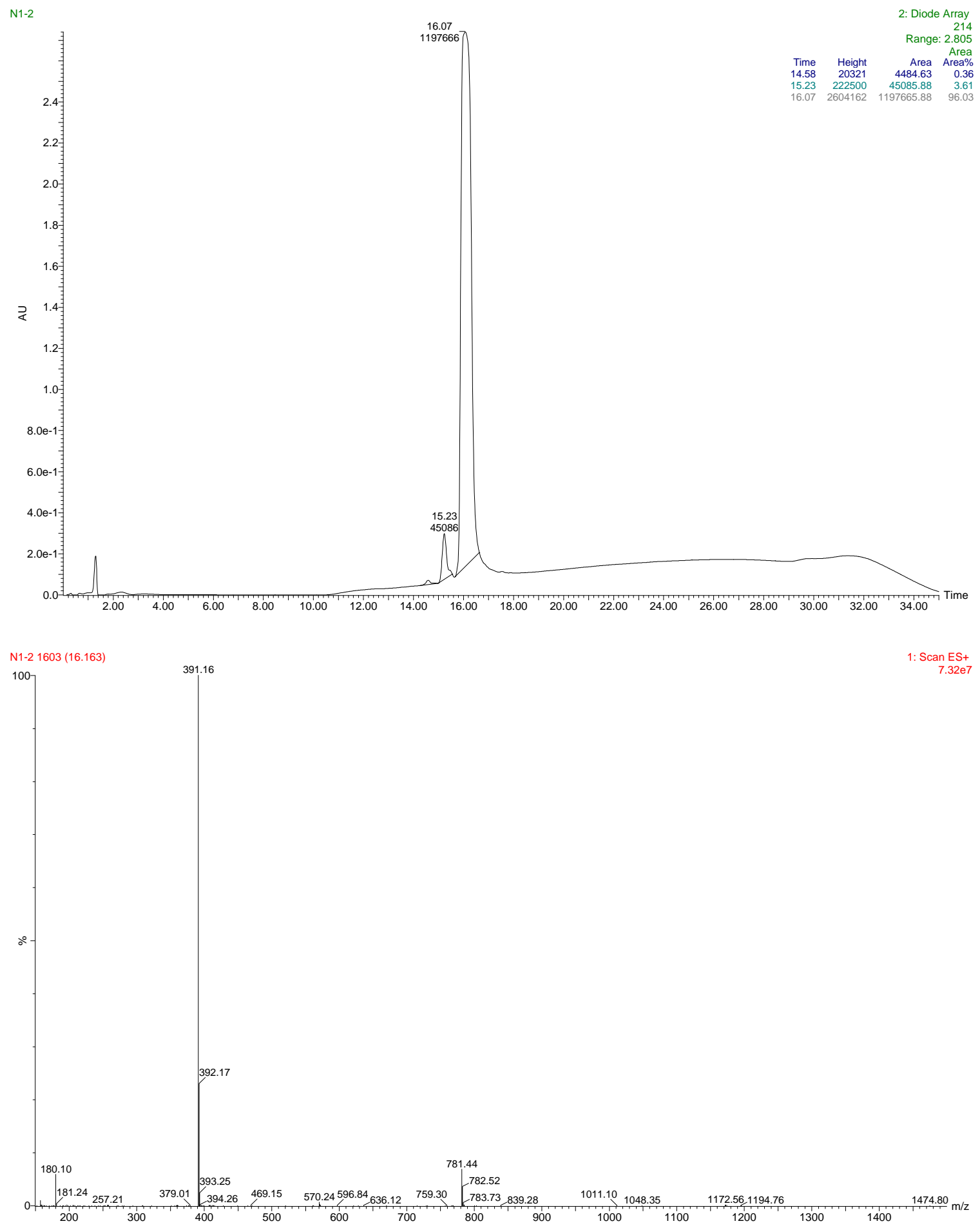




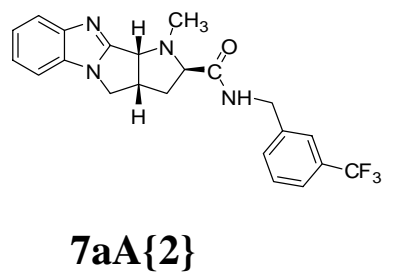

7aA $\{2\}$
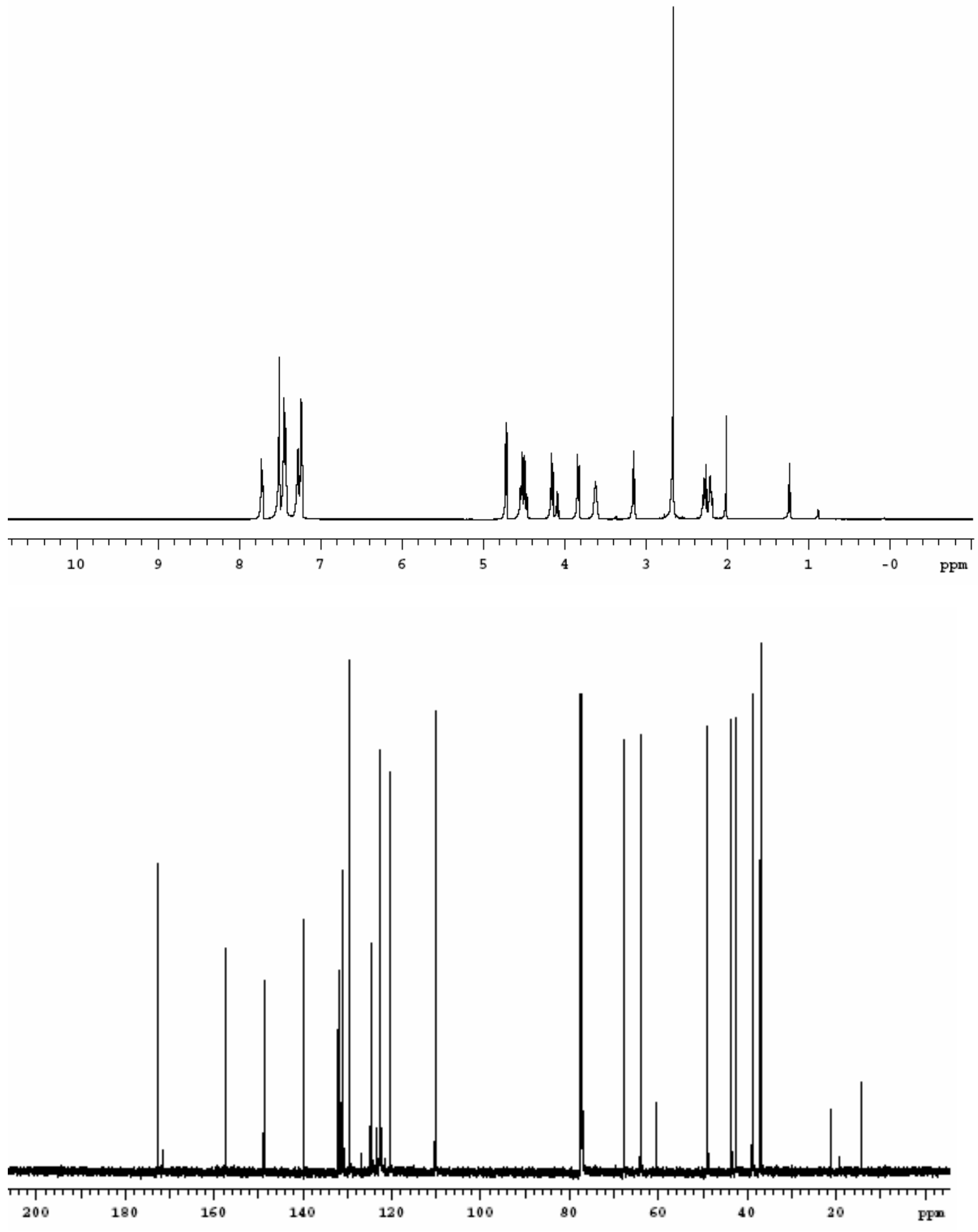

S45 


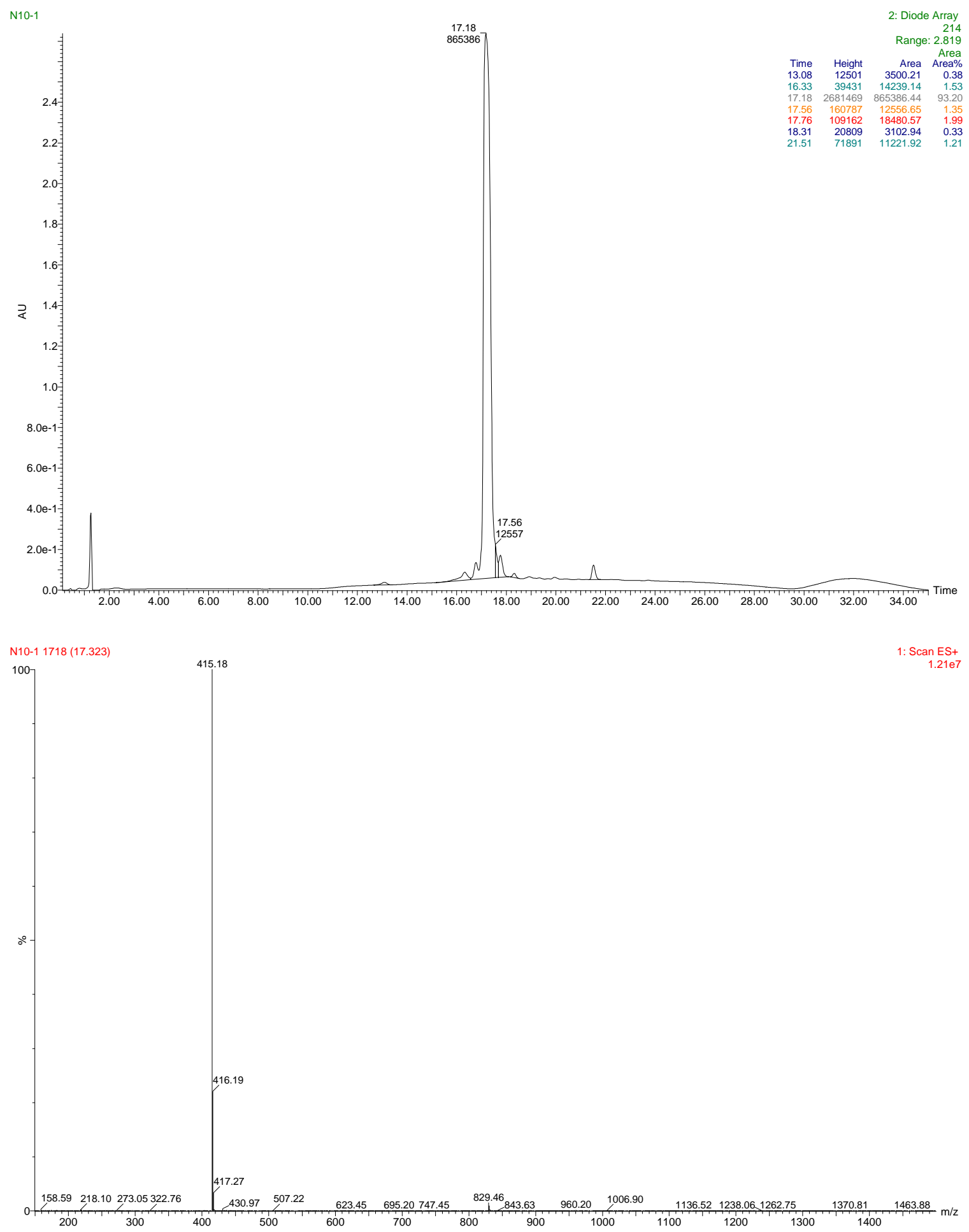




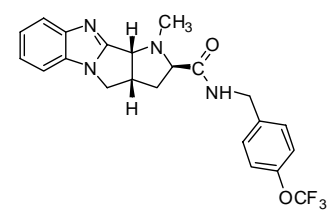

7aA $\{3\}$
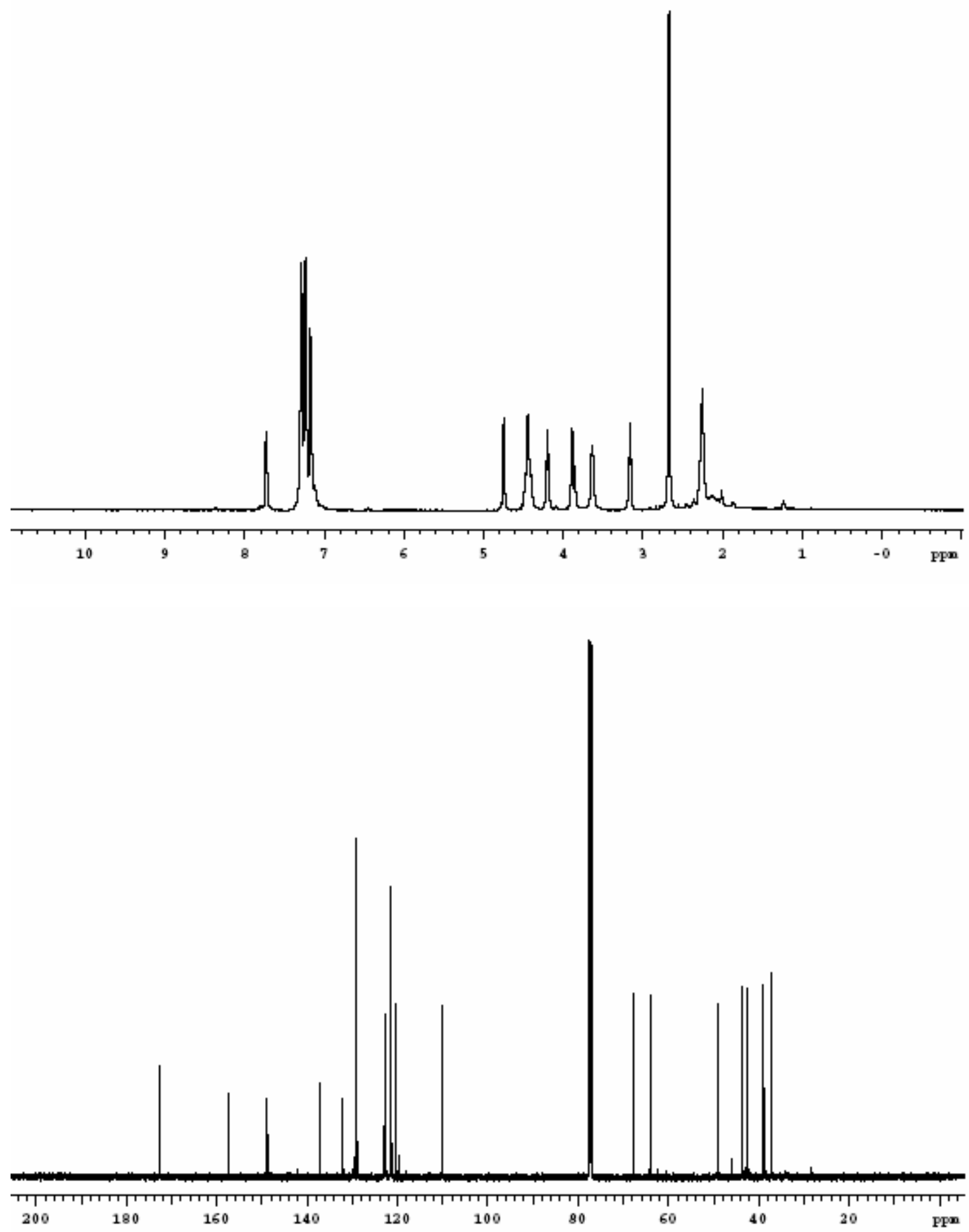
N5
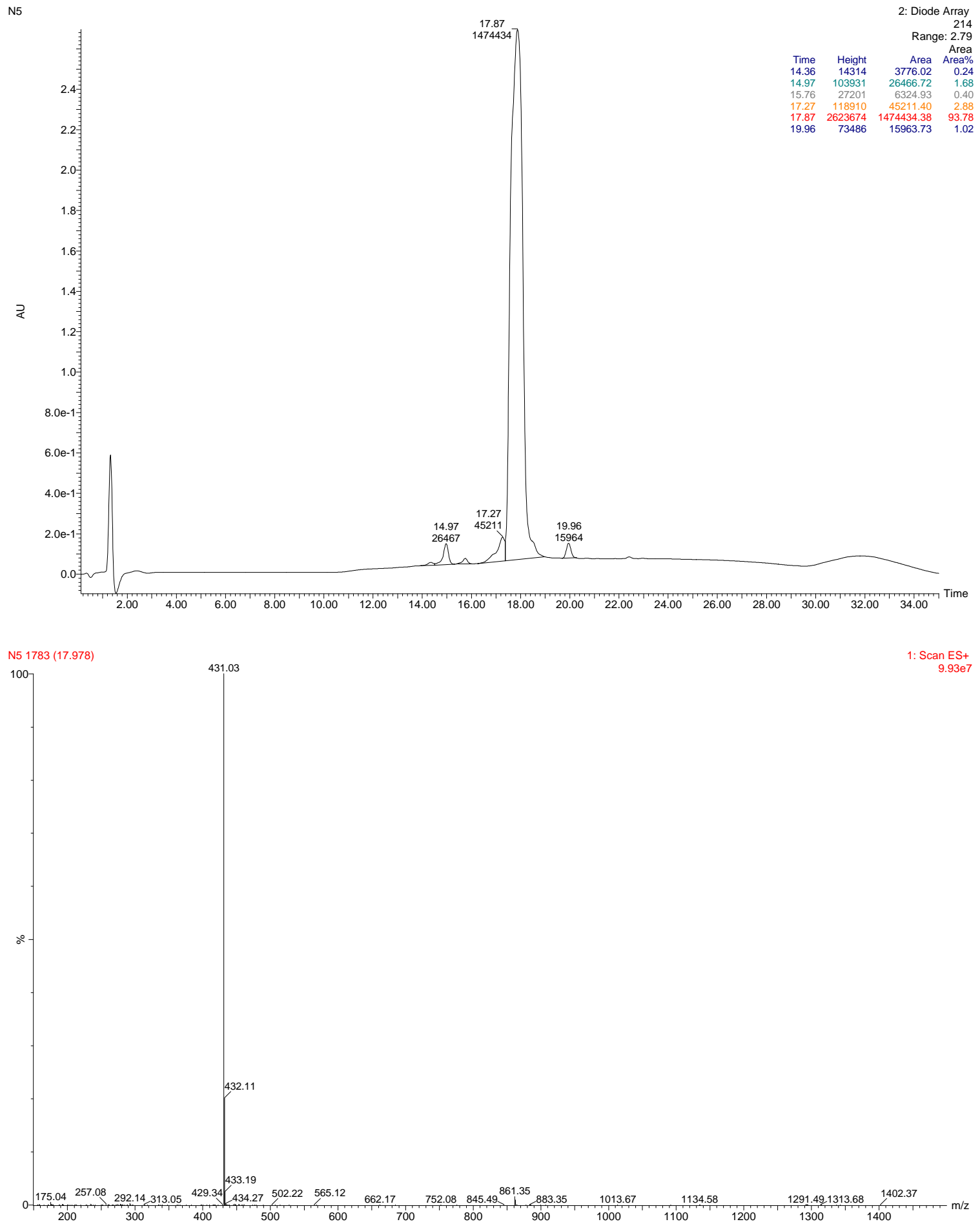


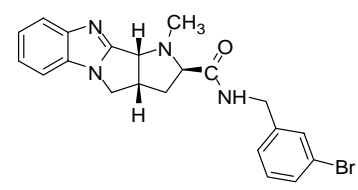

7aA $\{4\}$
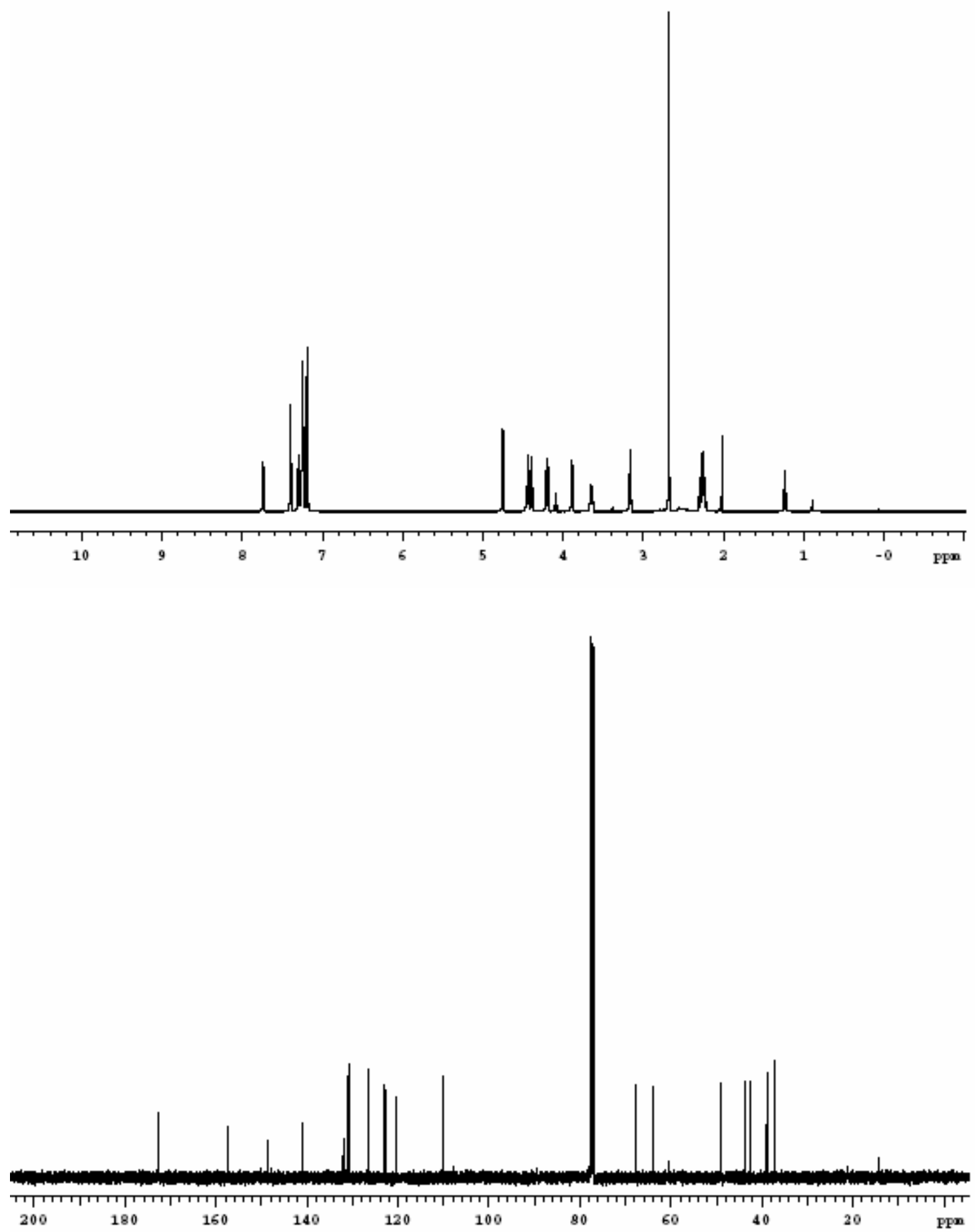
N3
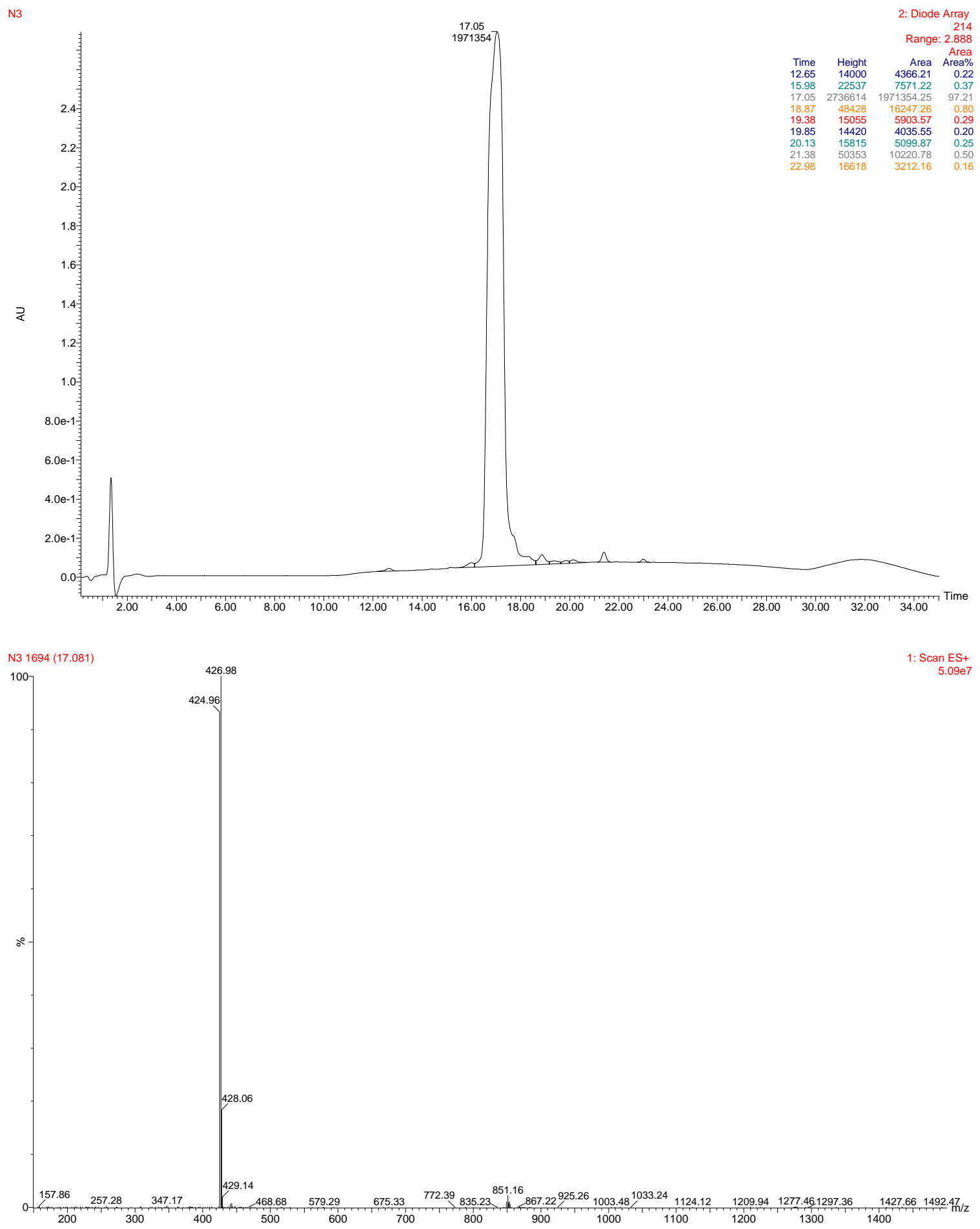

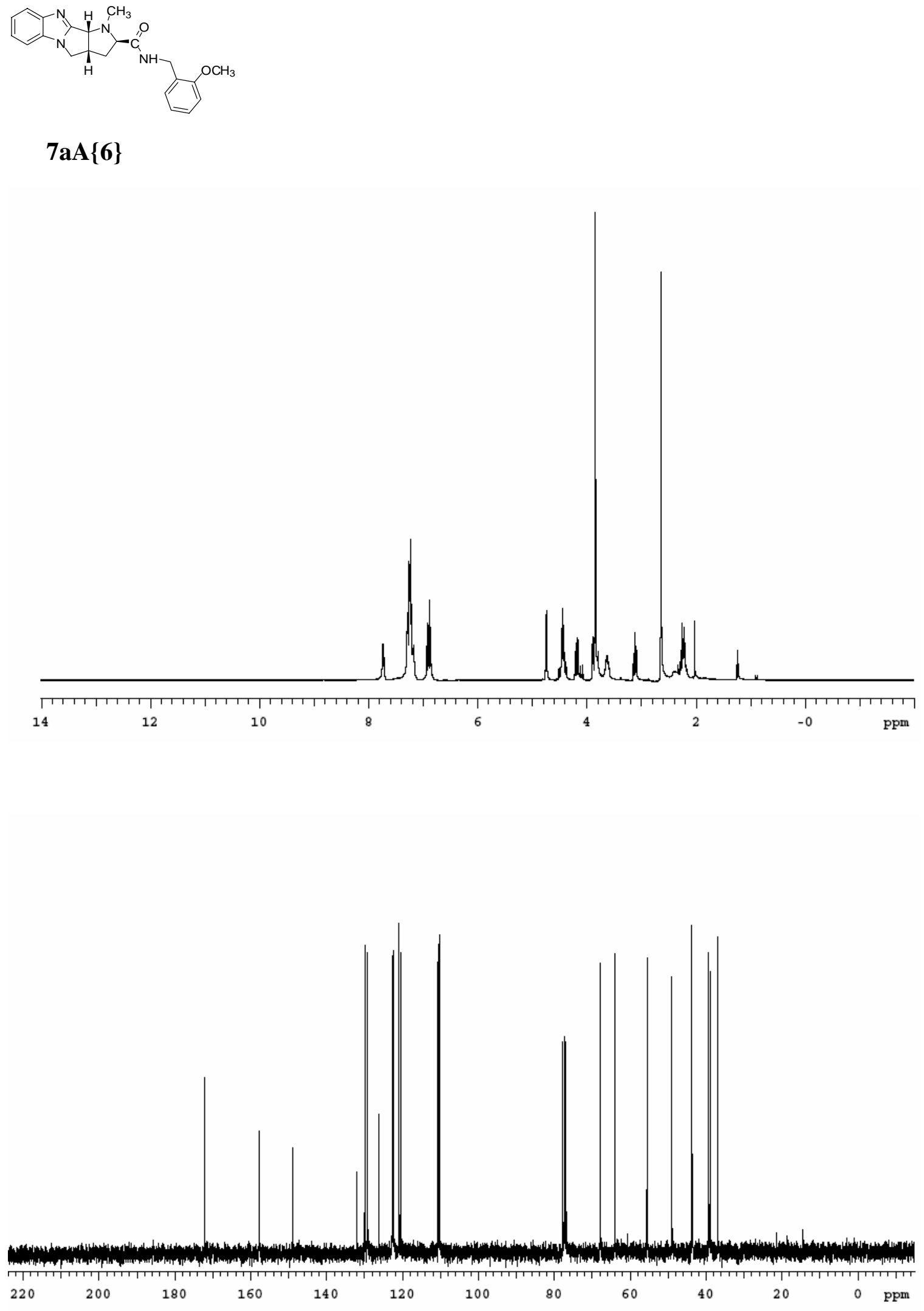

S51 


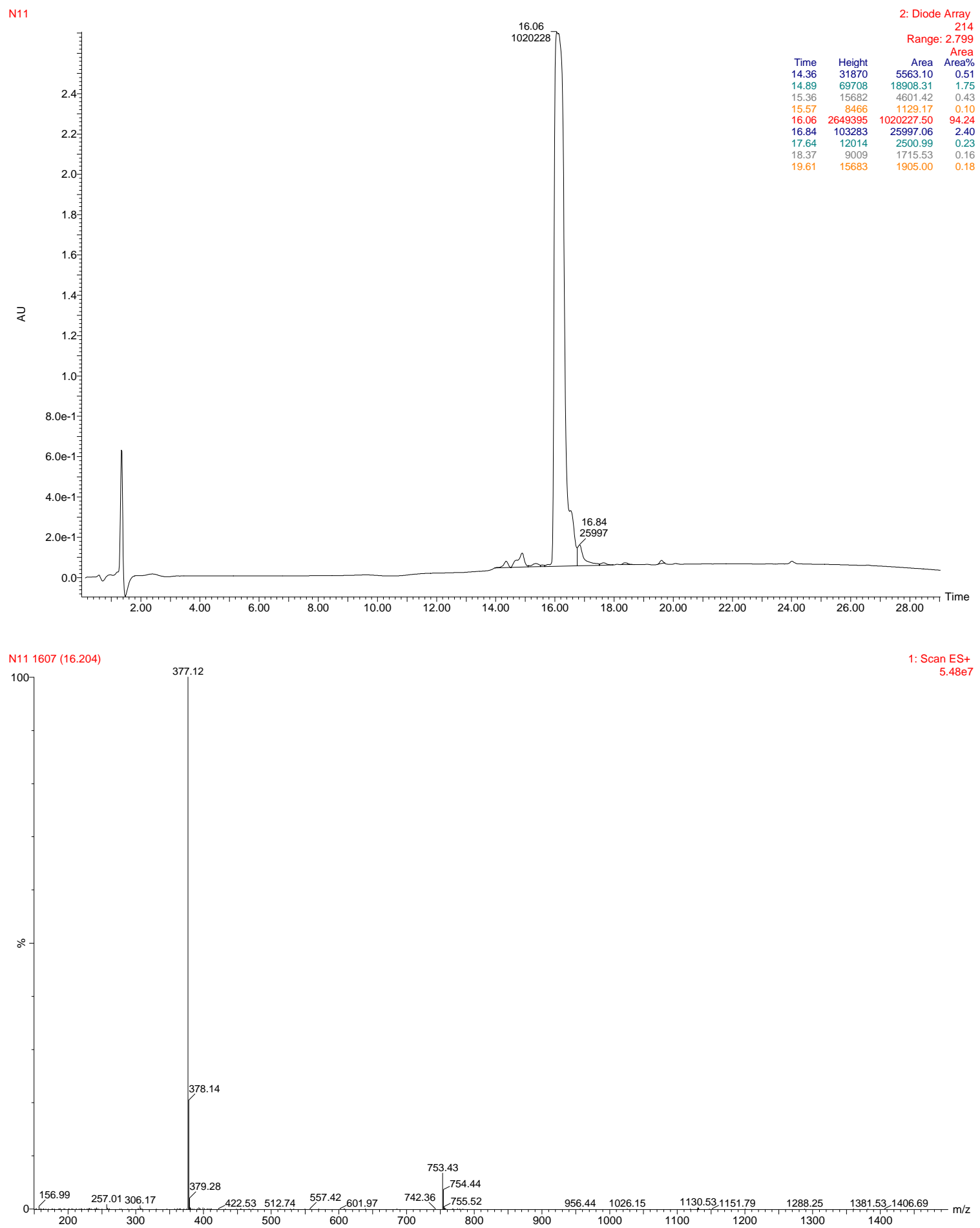



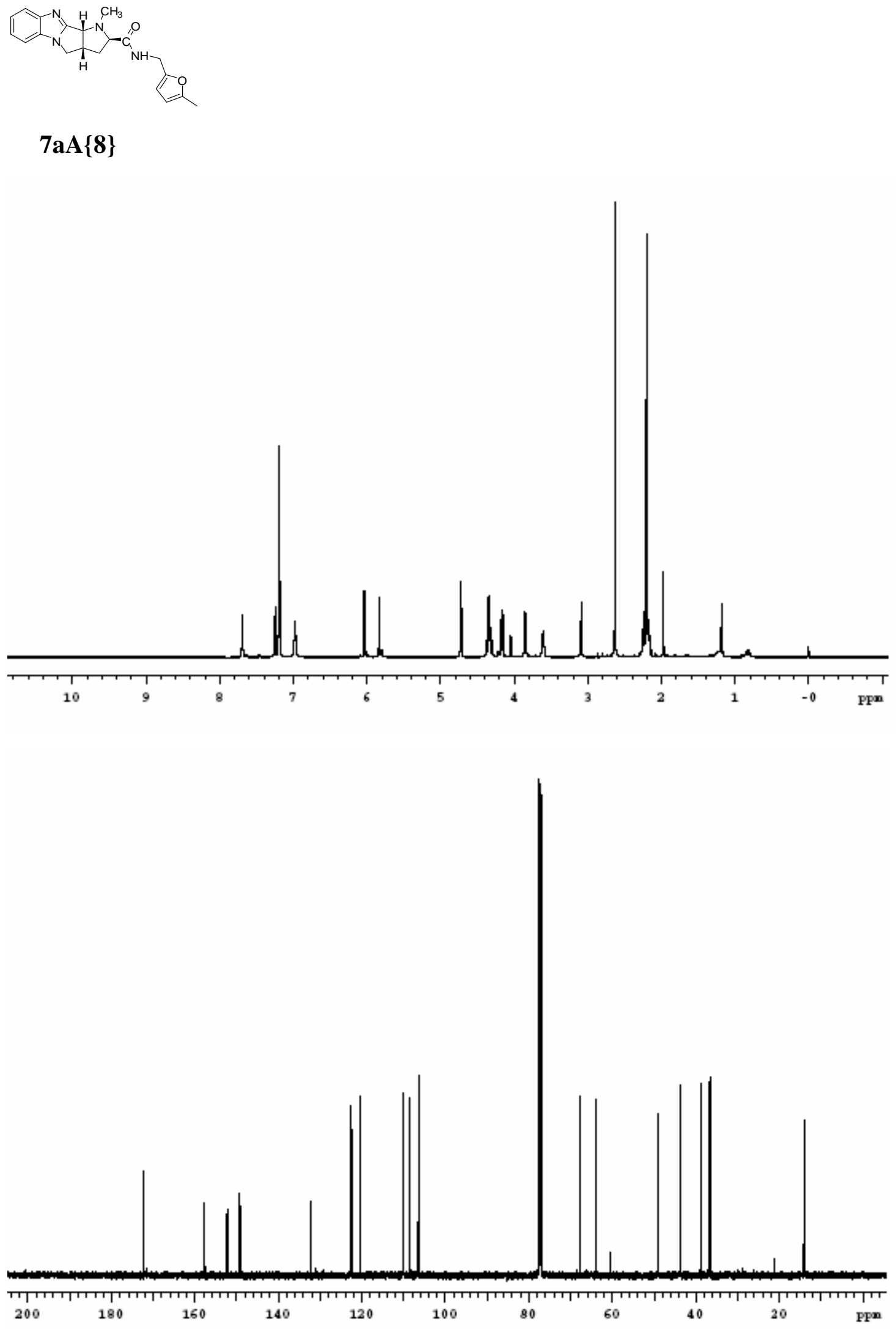

S53 

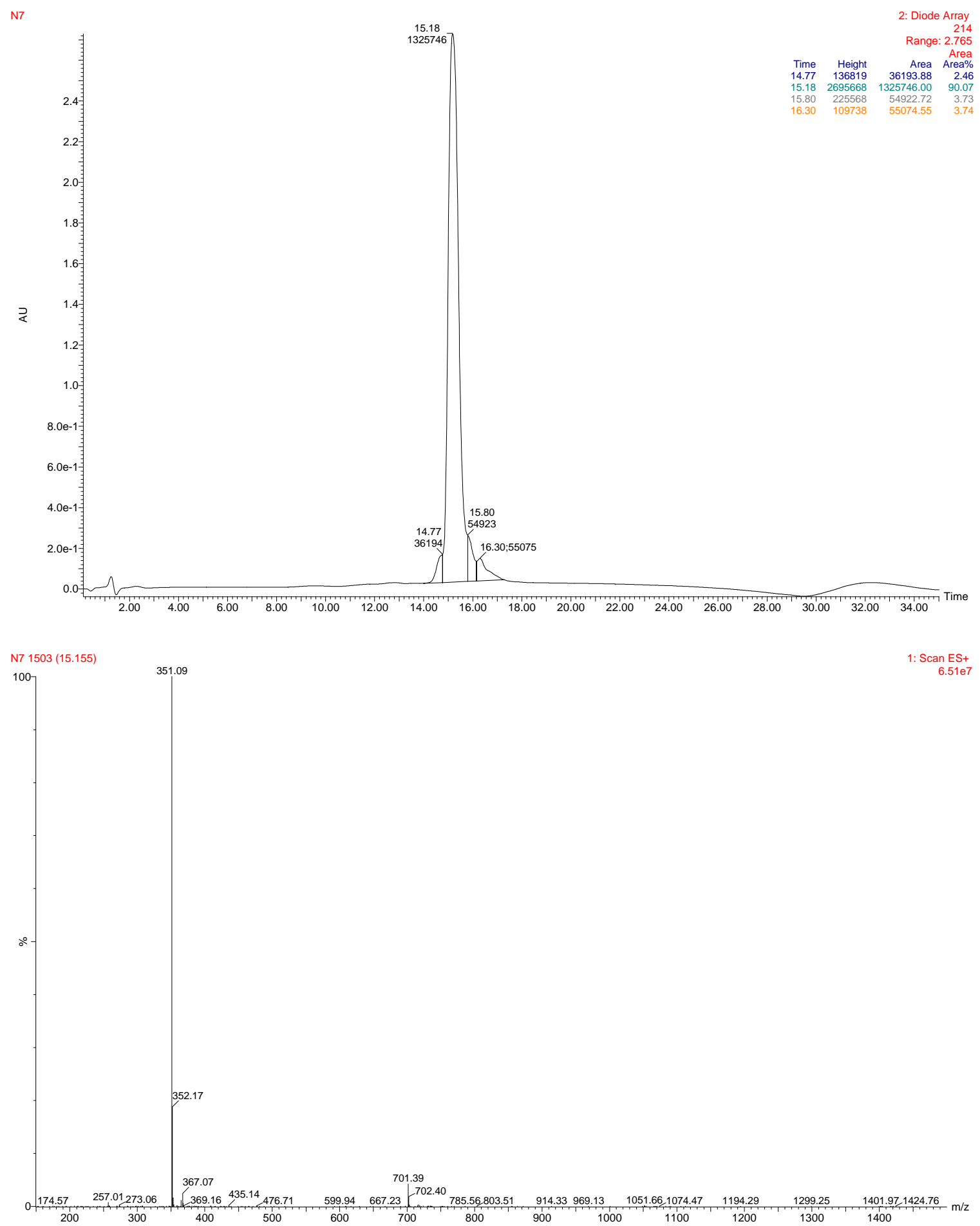

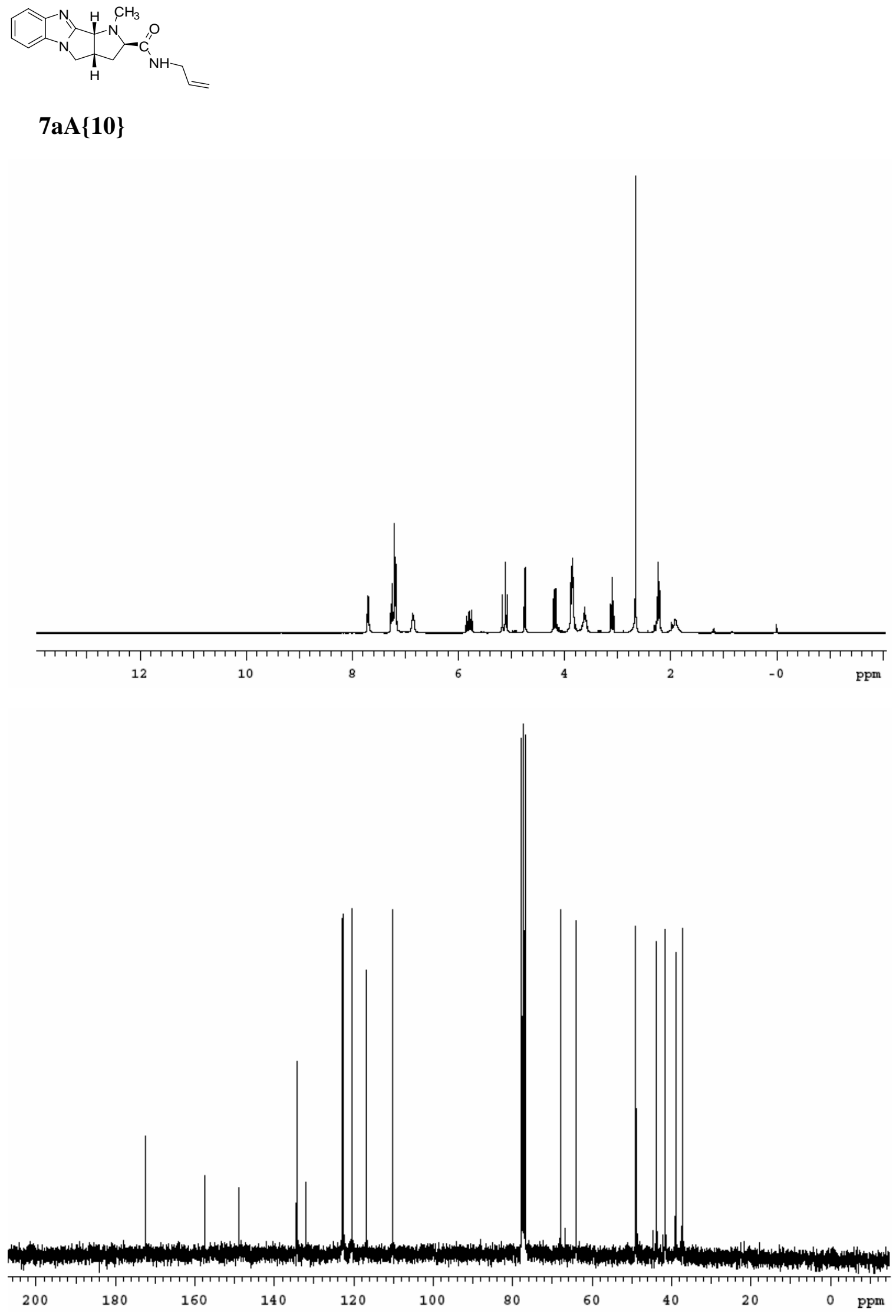

S55 

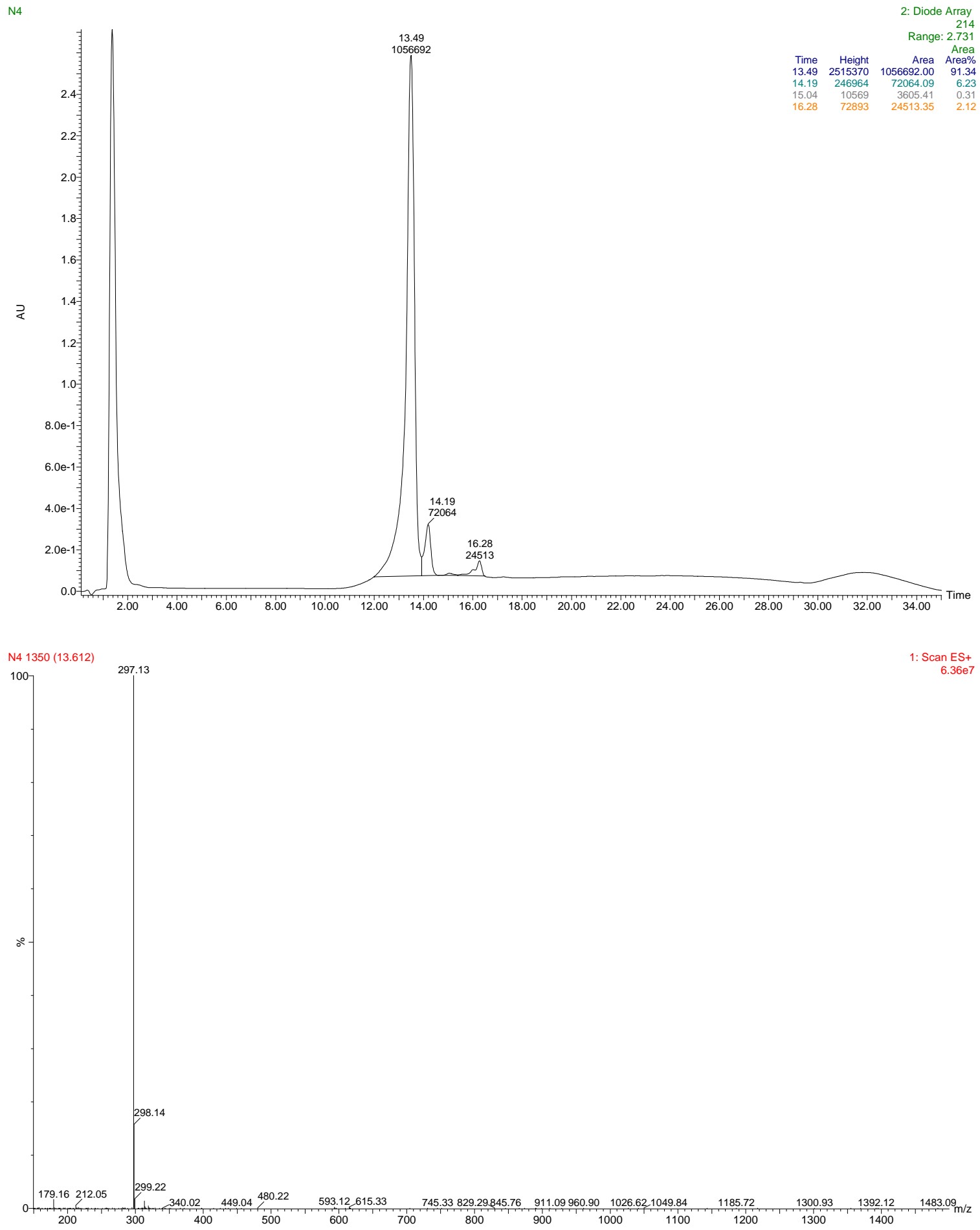


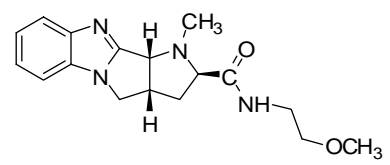

7aA $\{11\}$
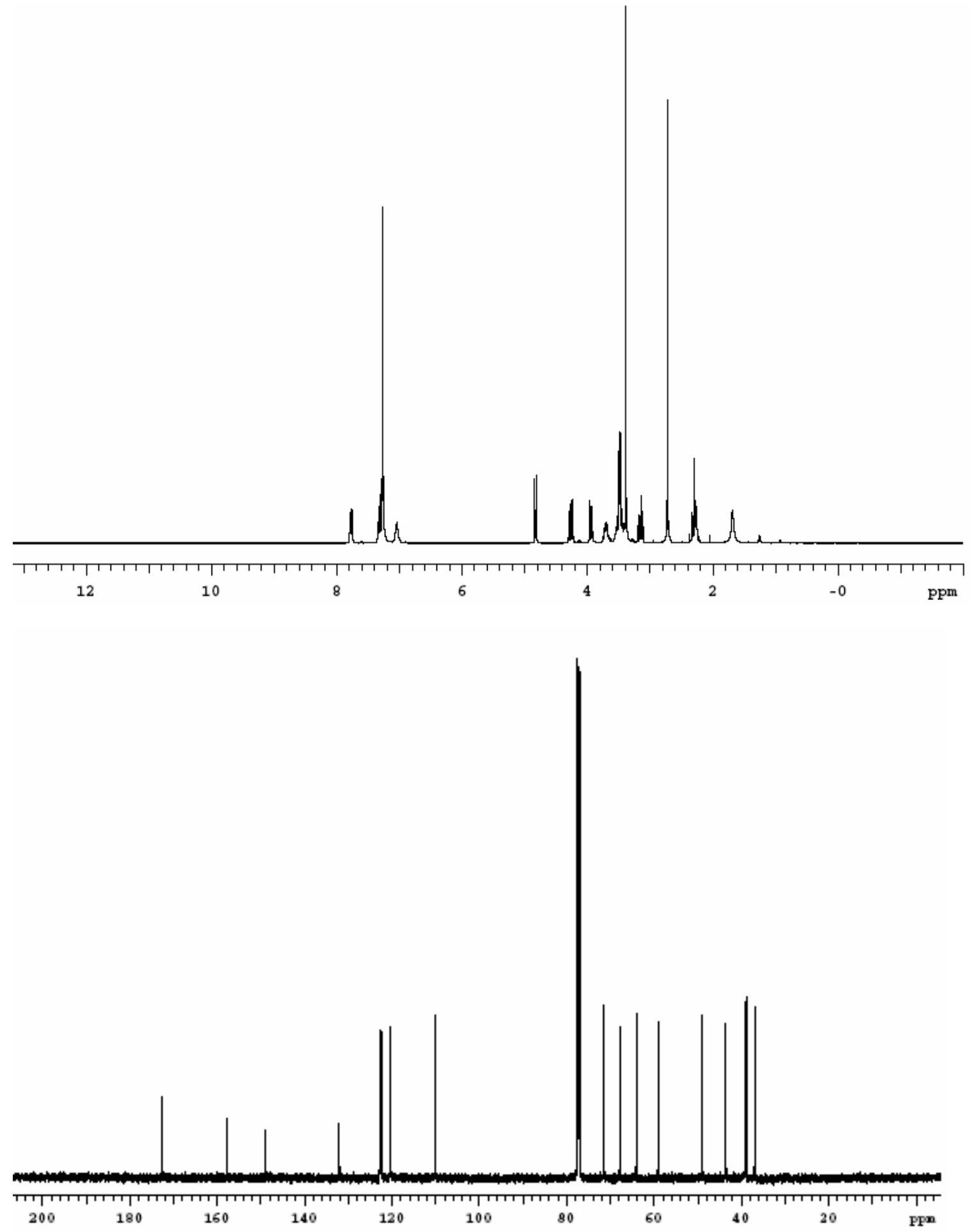

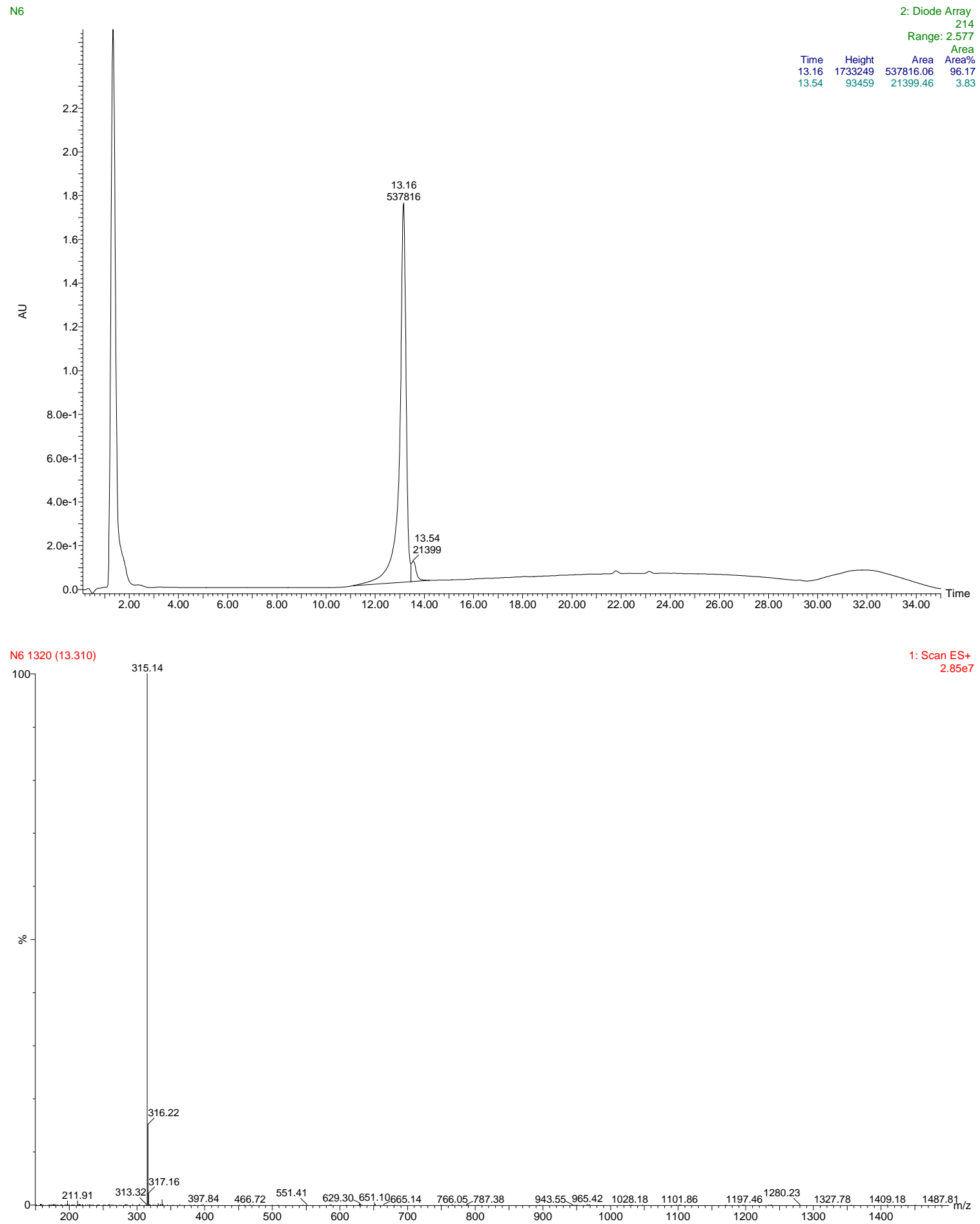

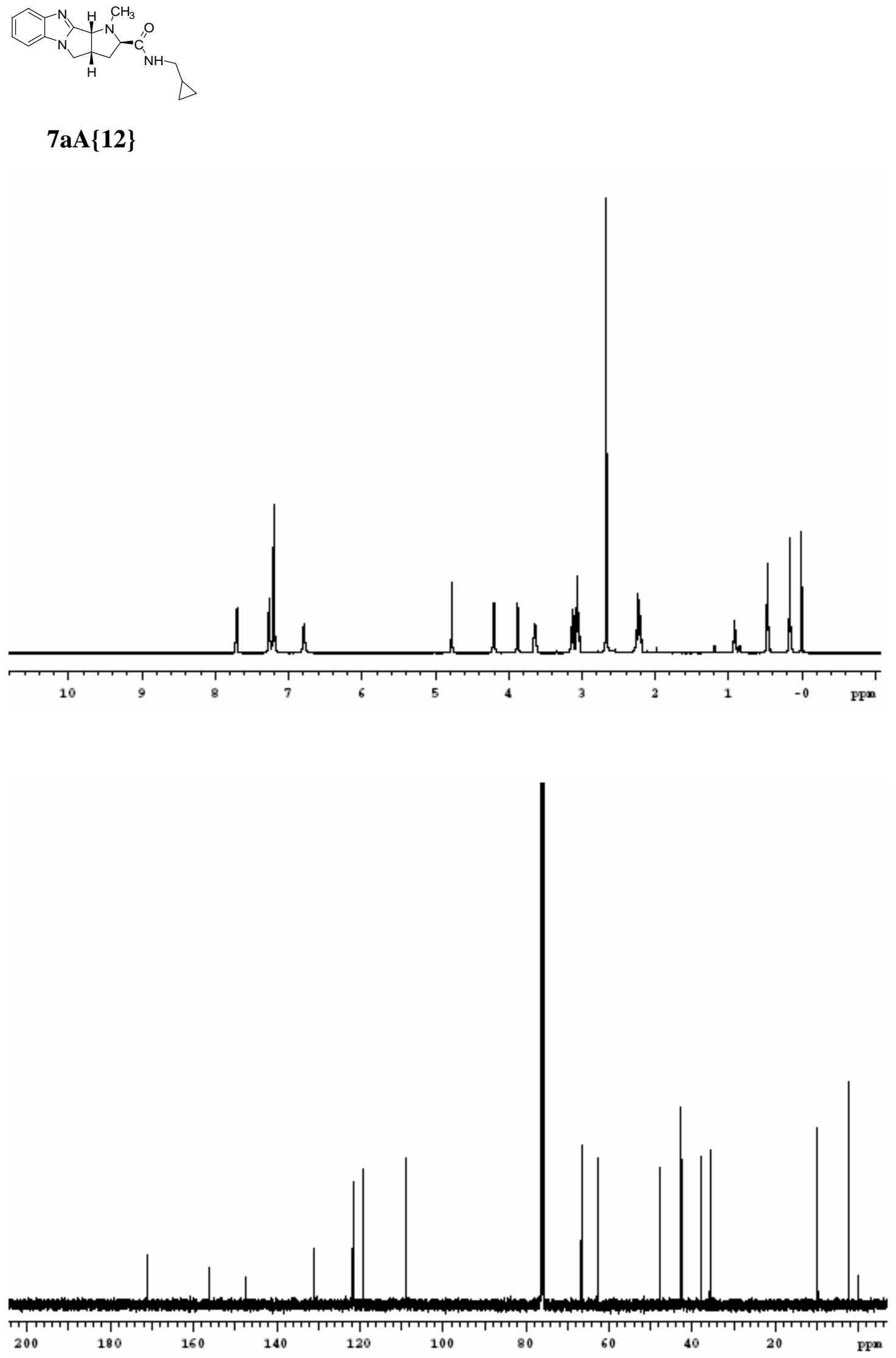

S59 


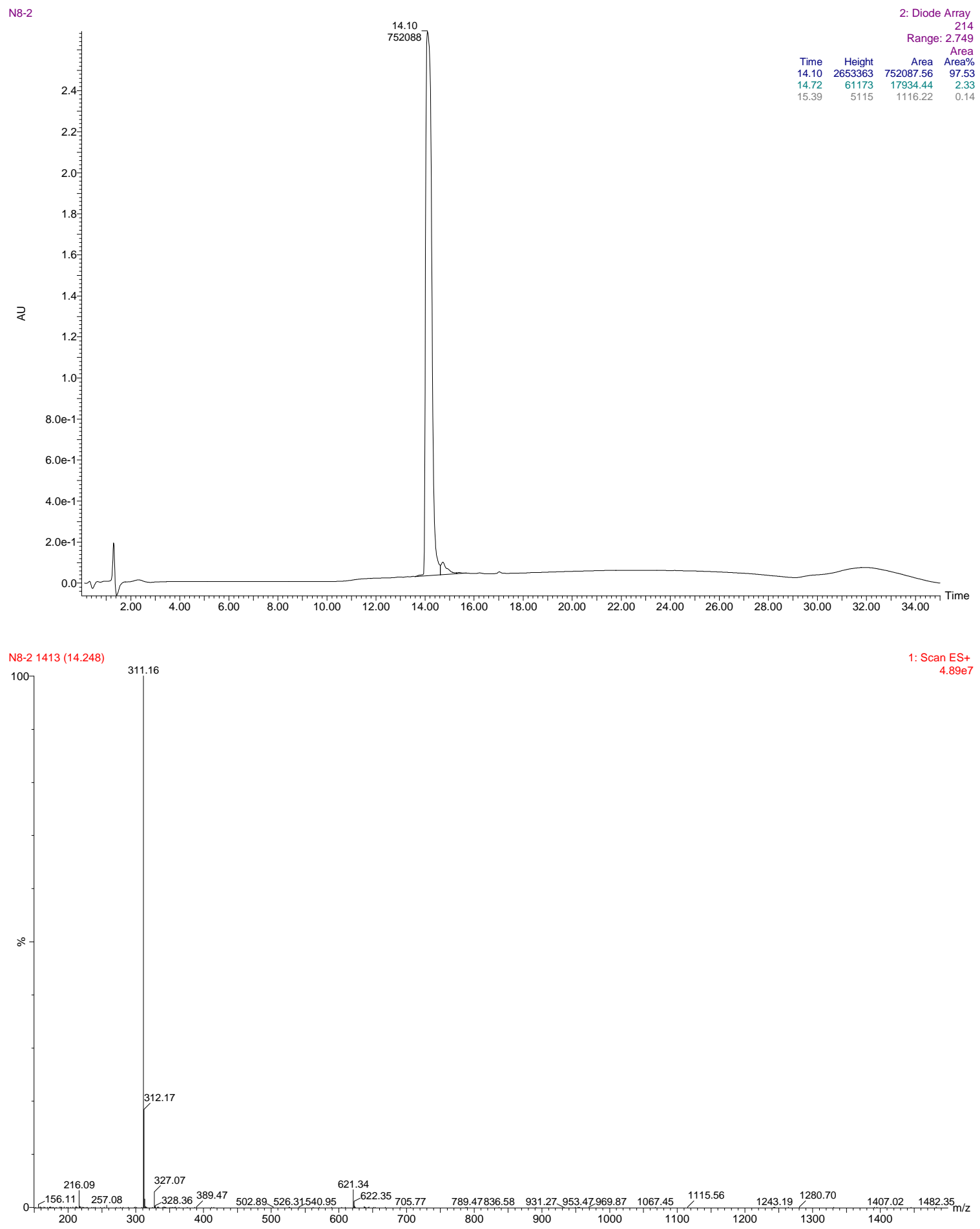



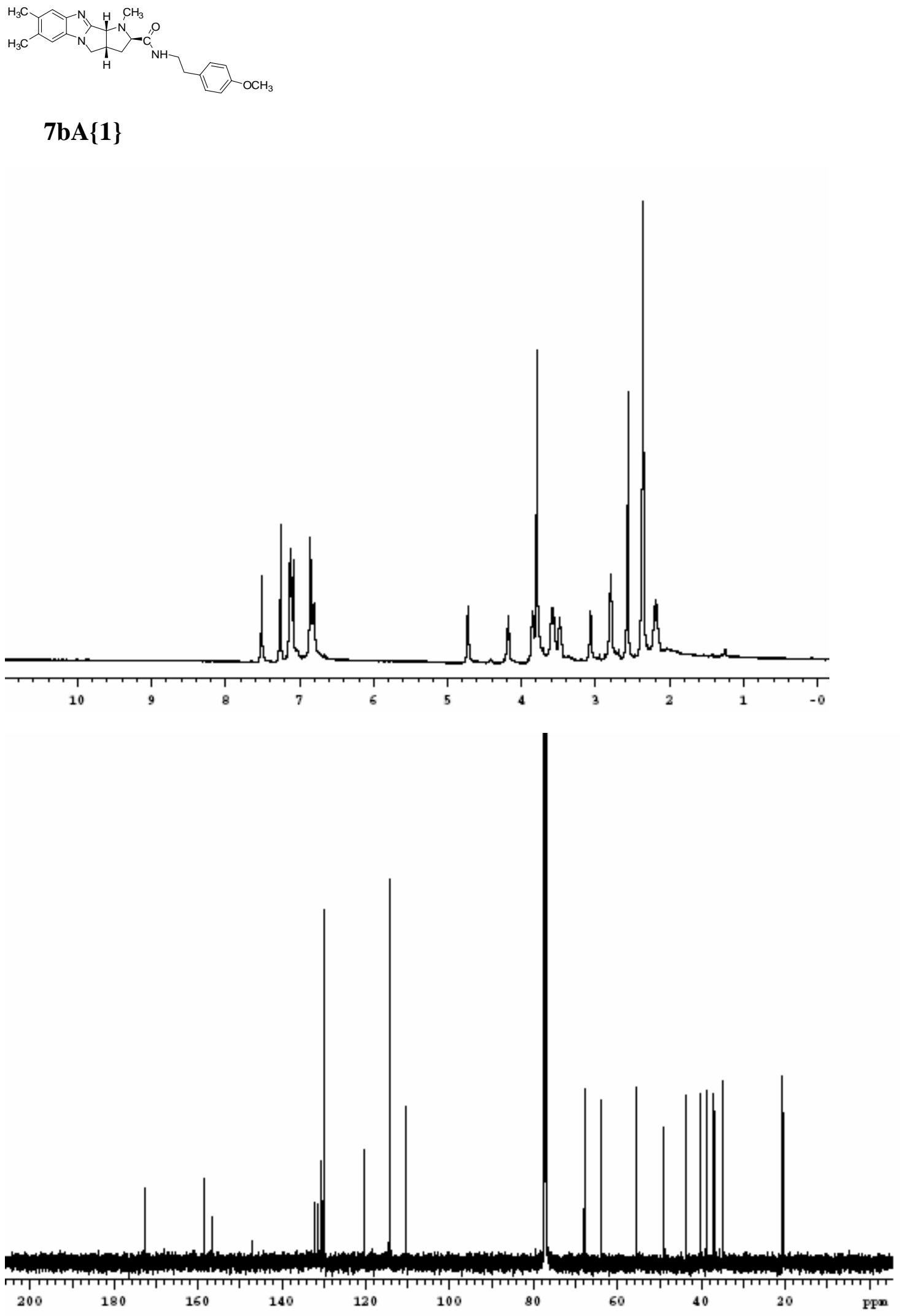

S61 


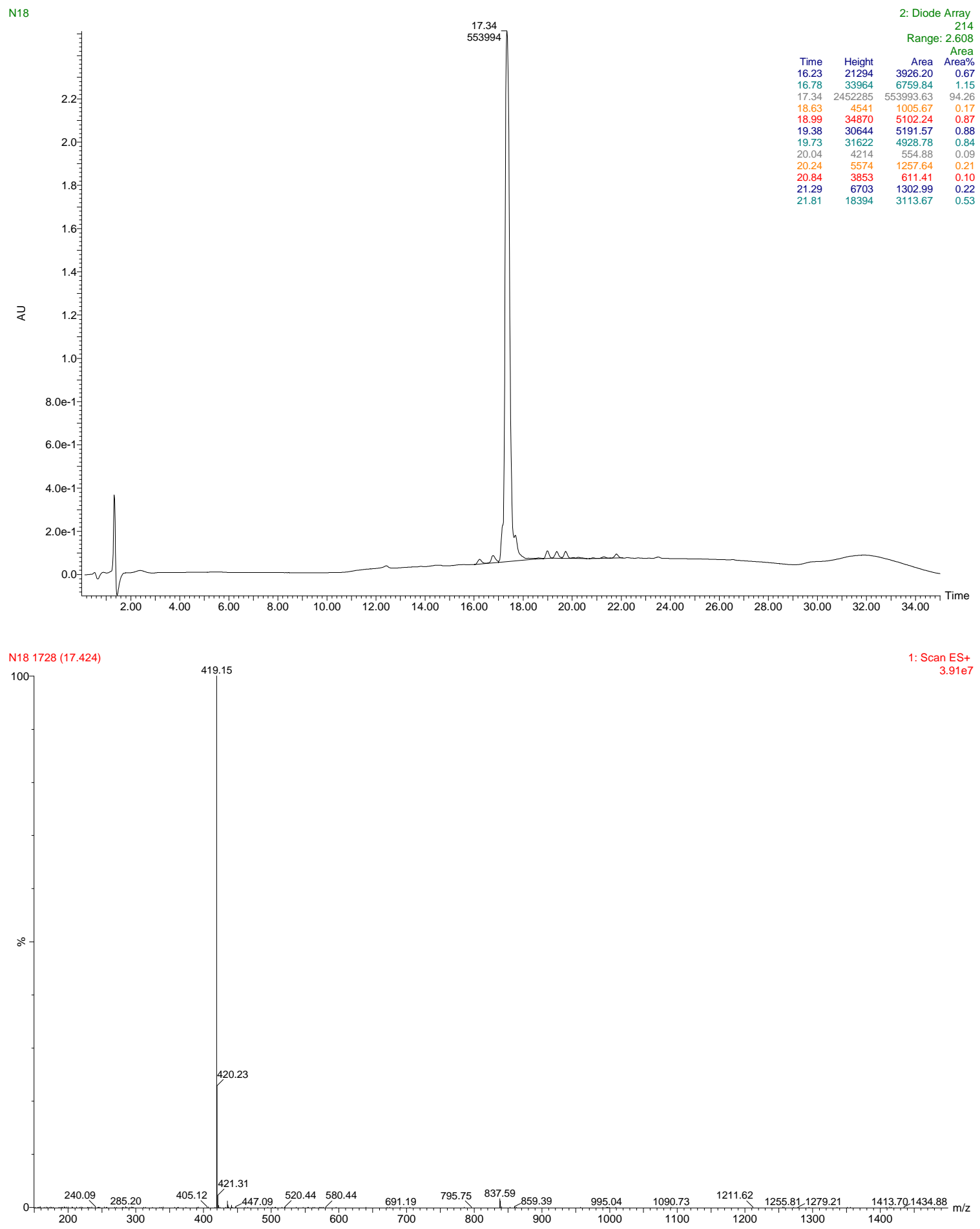



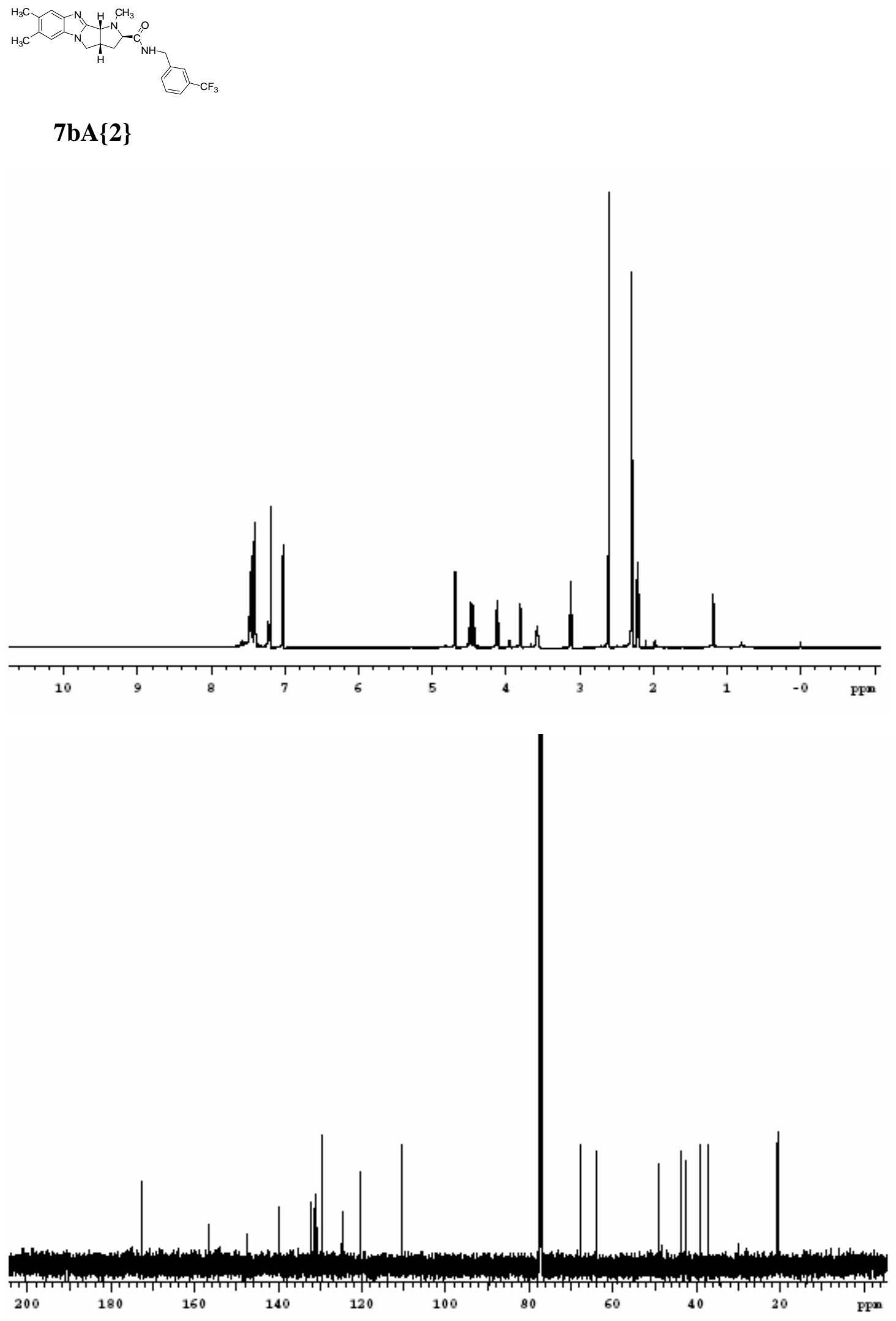

S63 


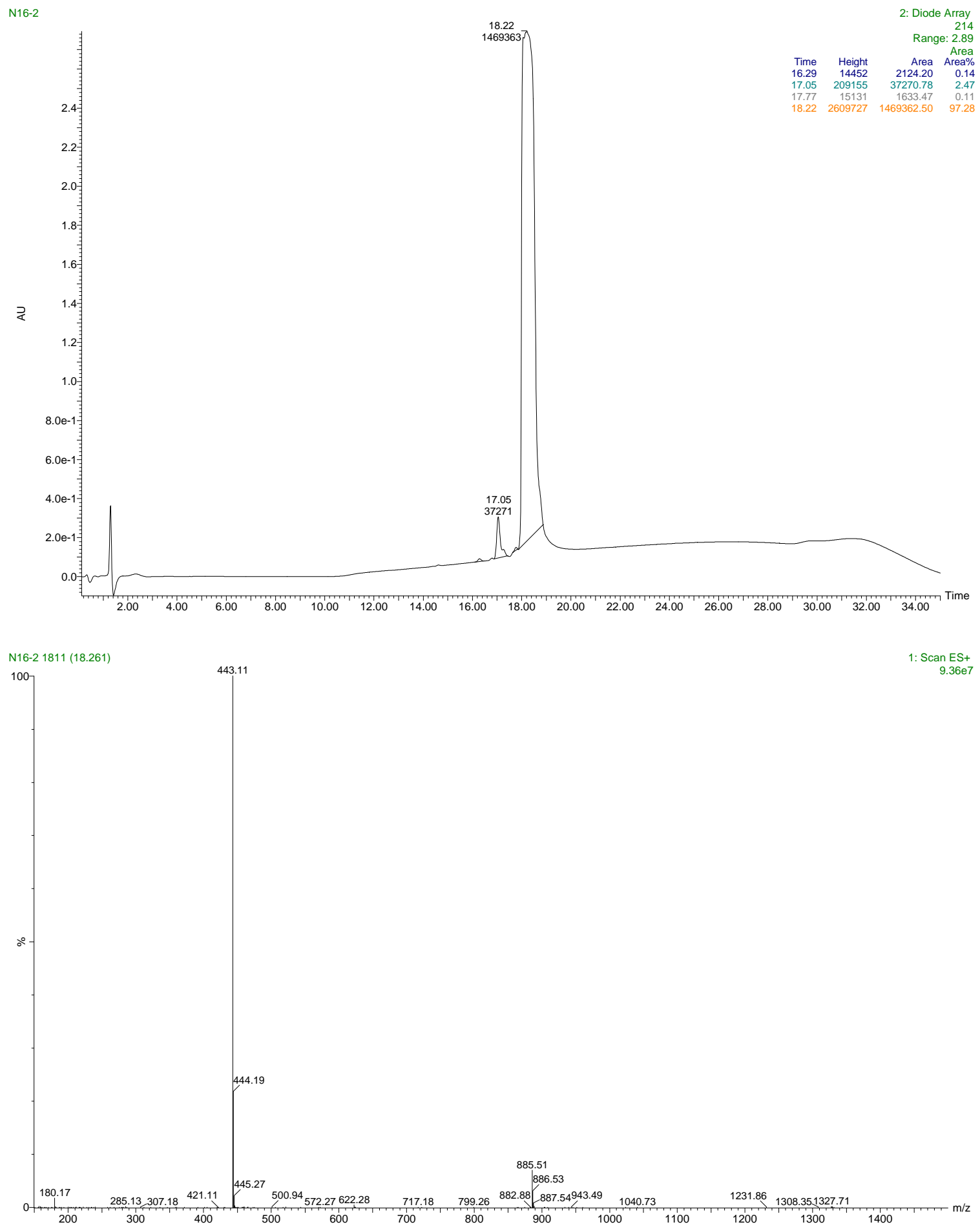




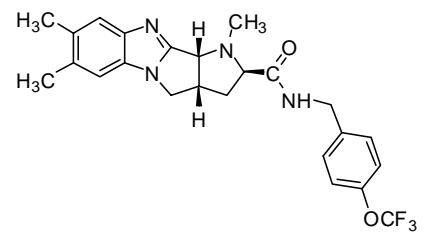

7bA $\{3\}$
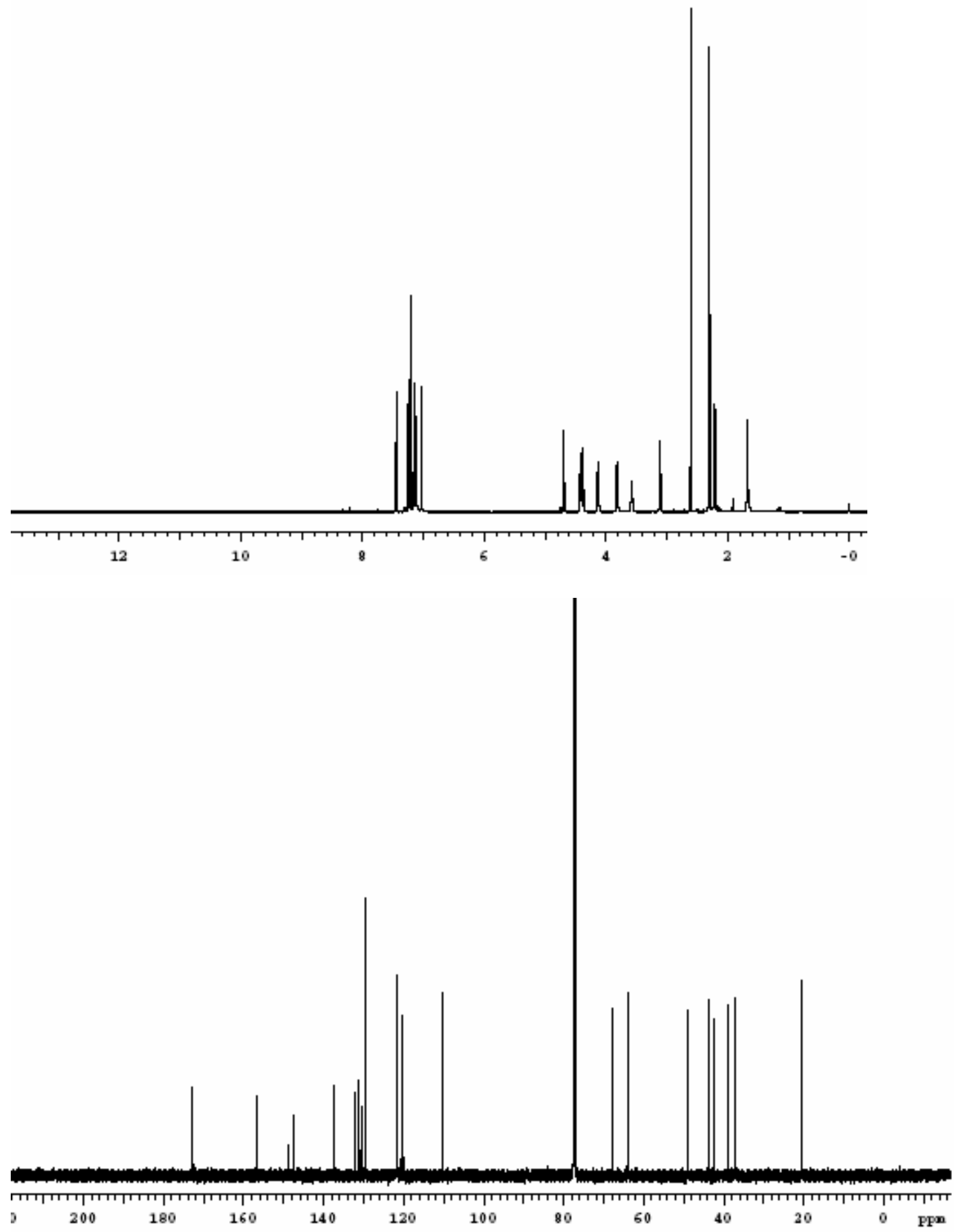


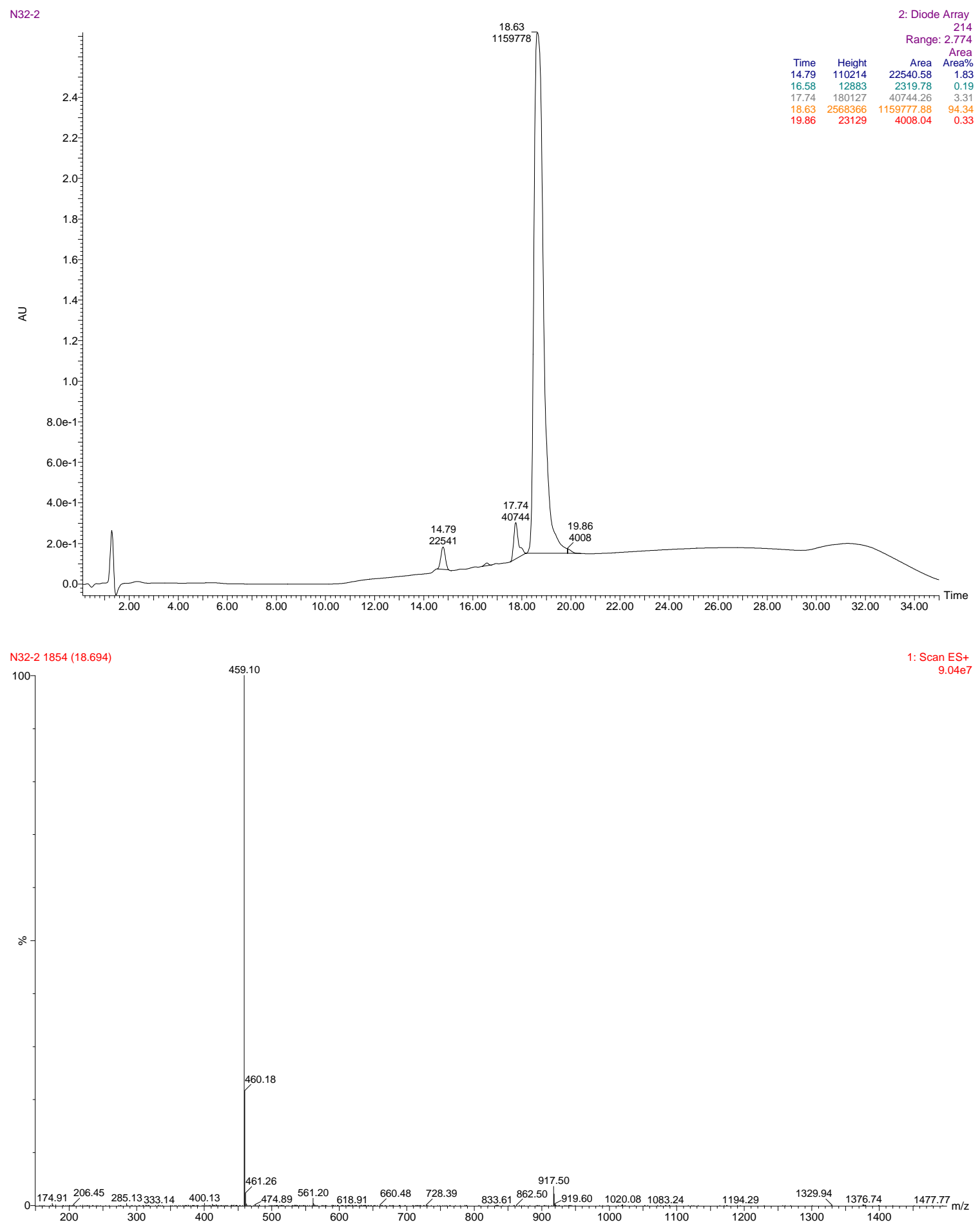




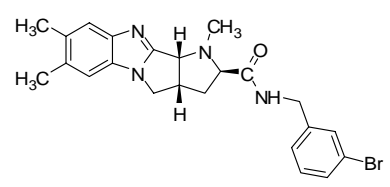

7bA $\{4\}$
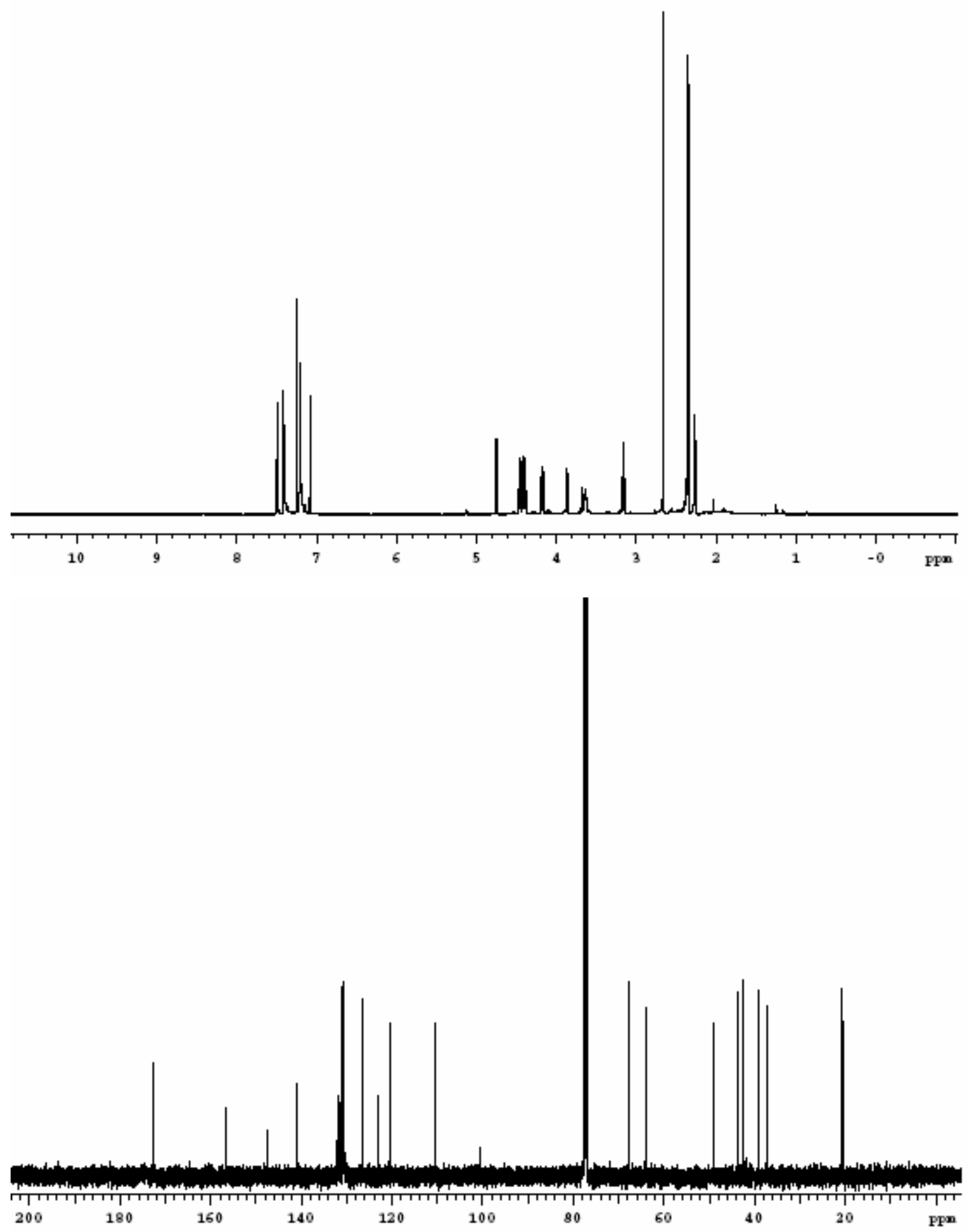


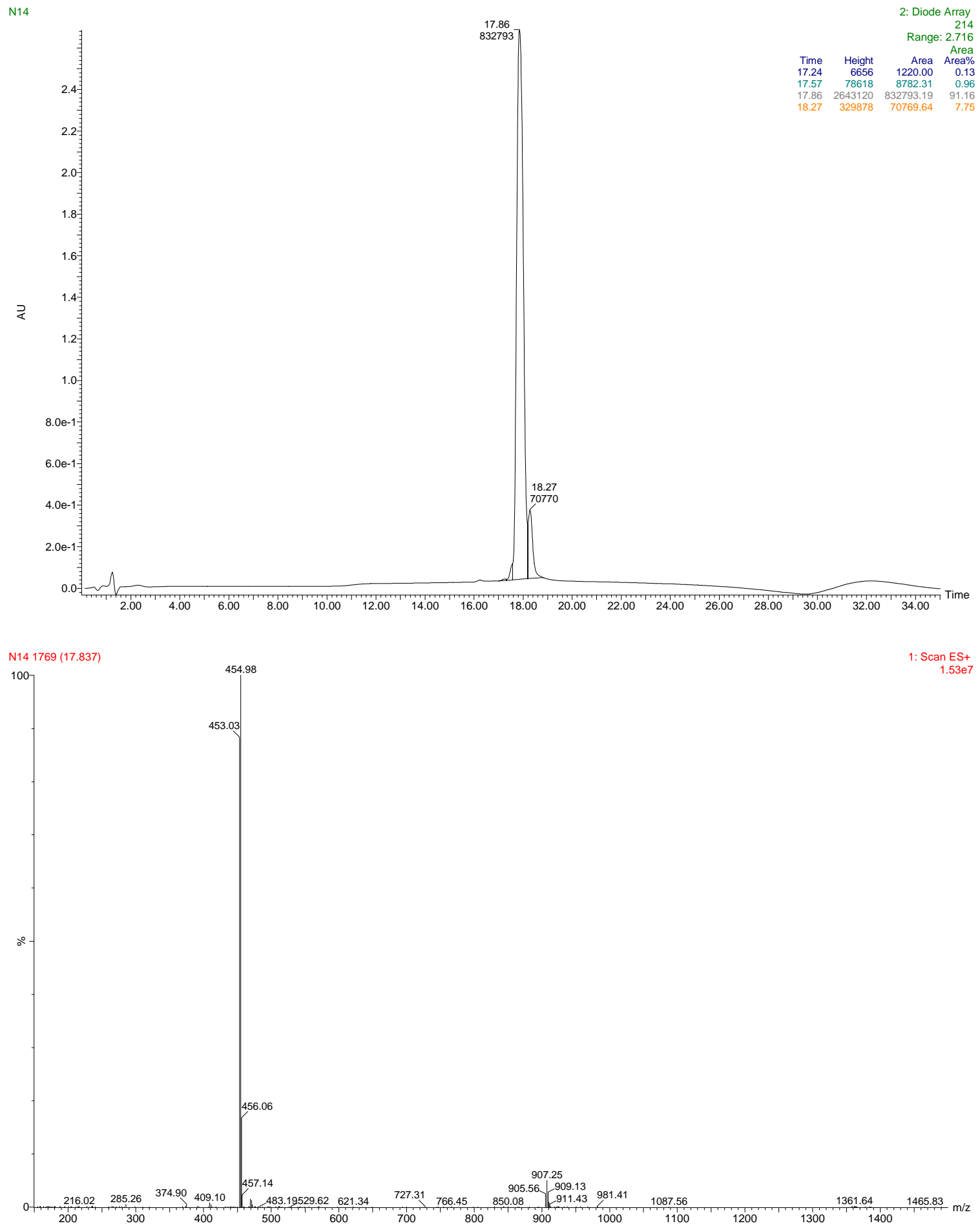




$$
\frac{1}{4}
$$




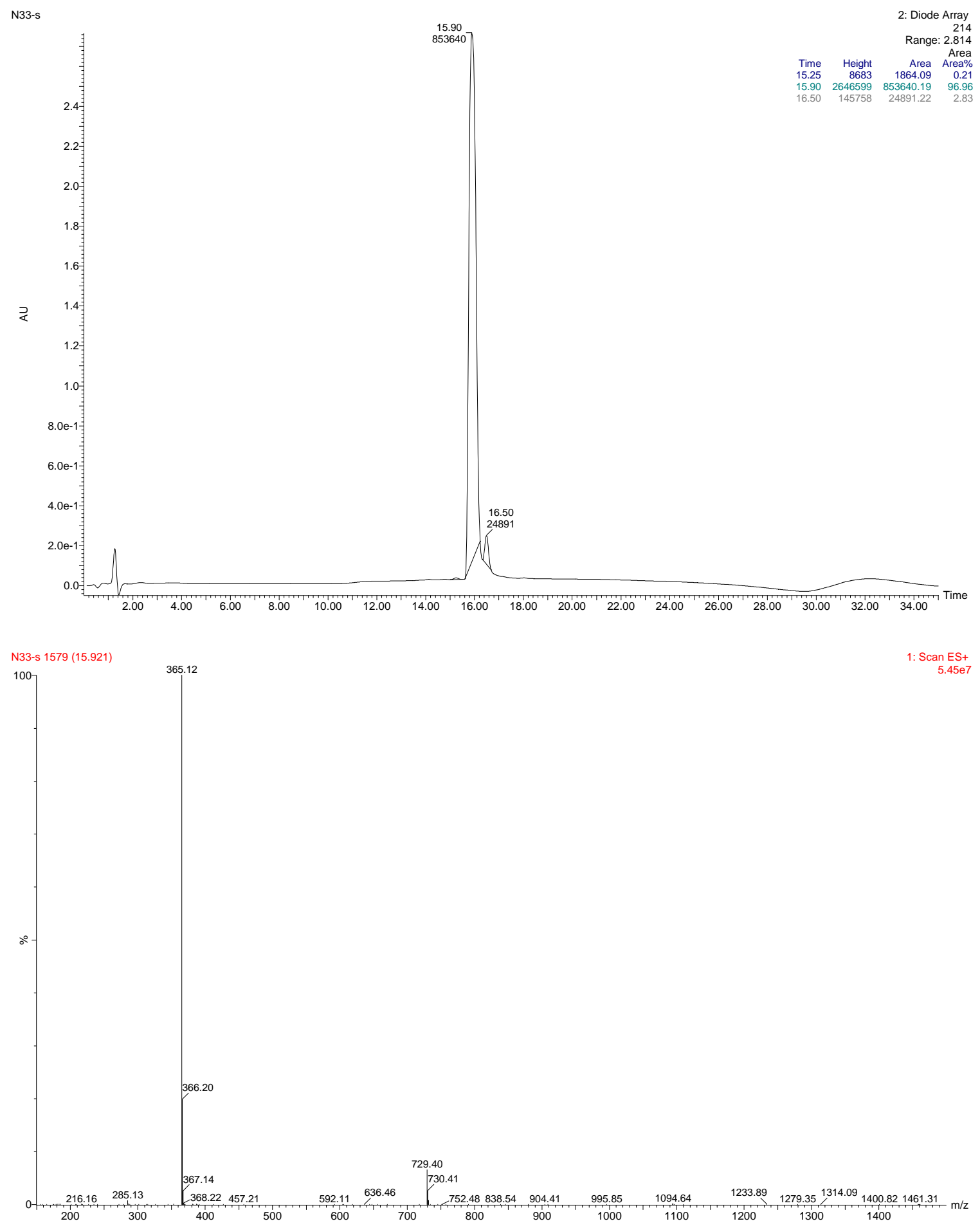




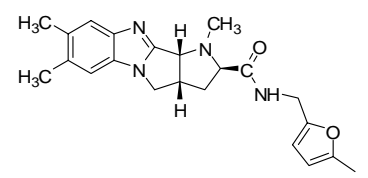

7bA $\{8\}$
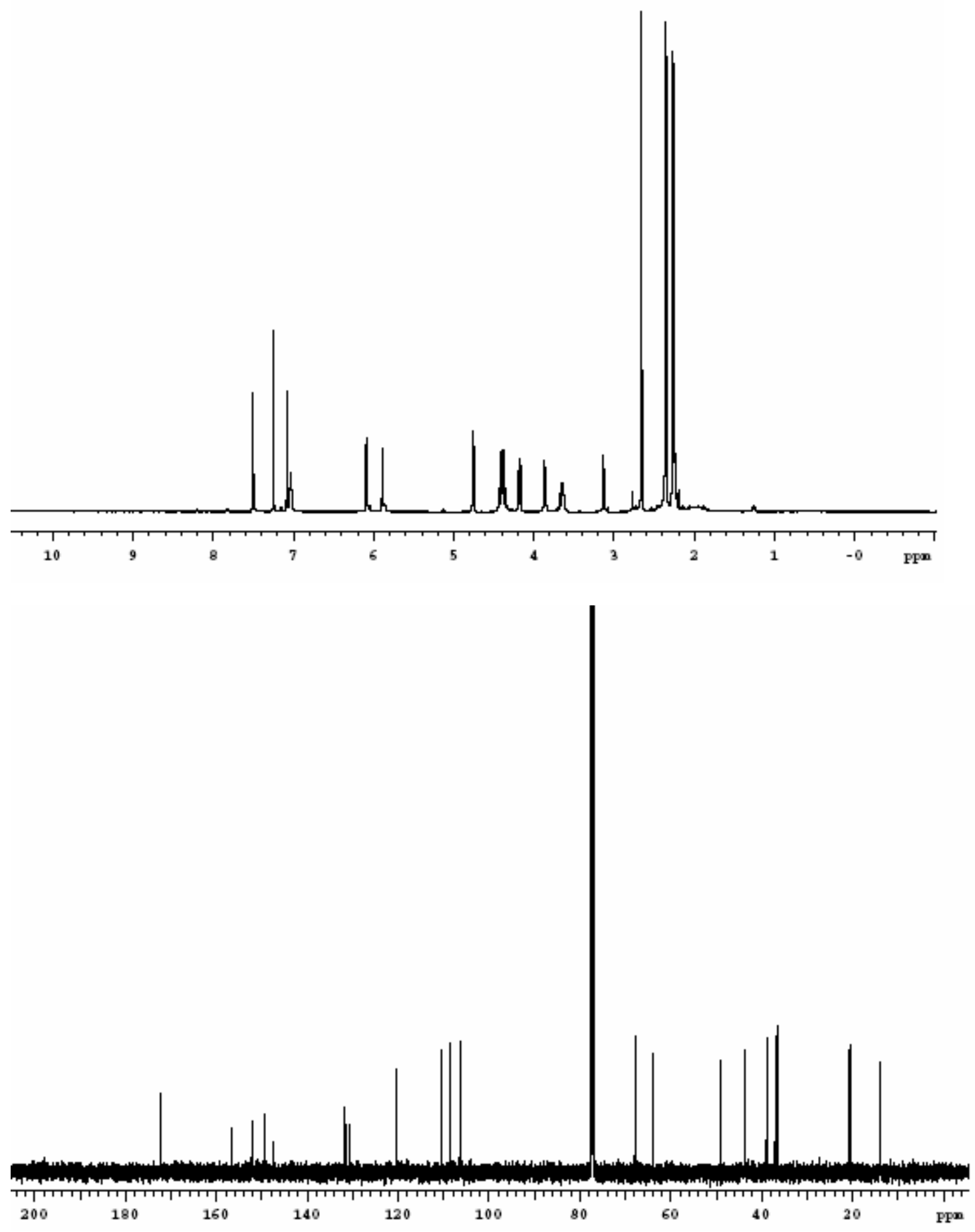

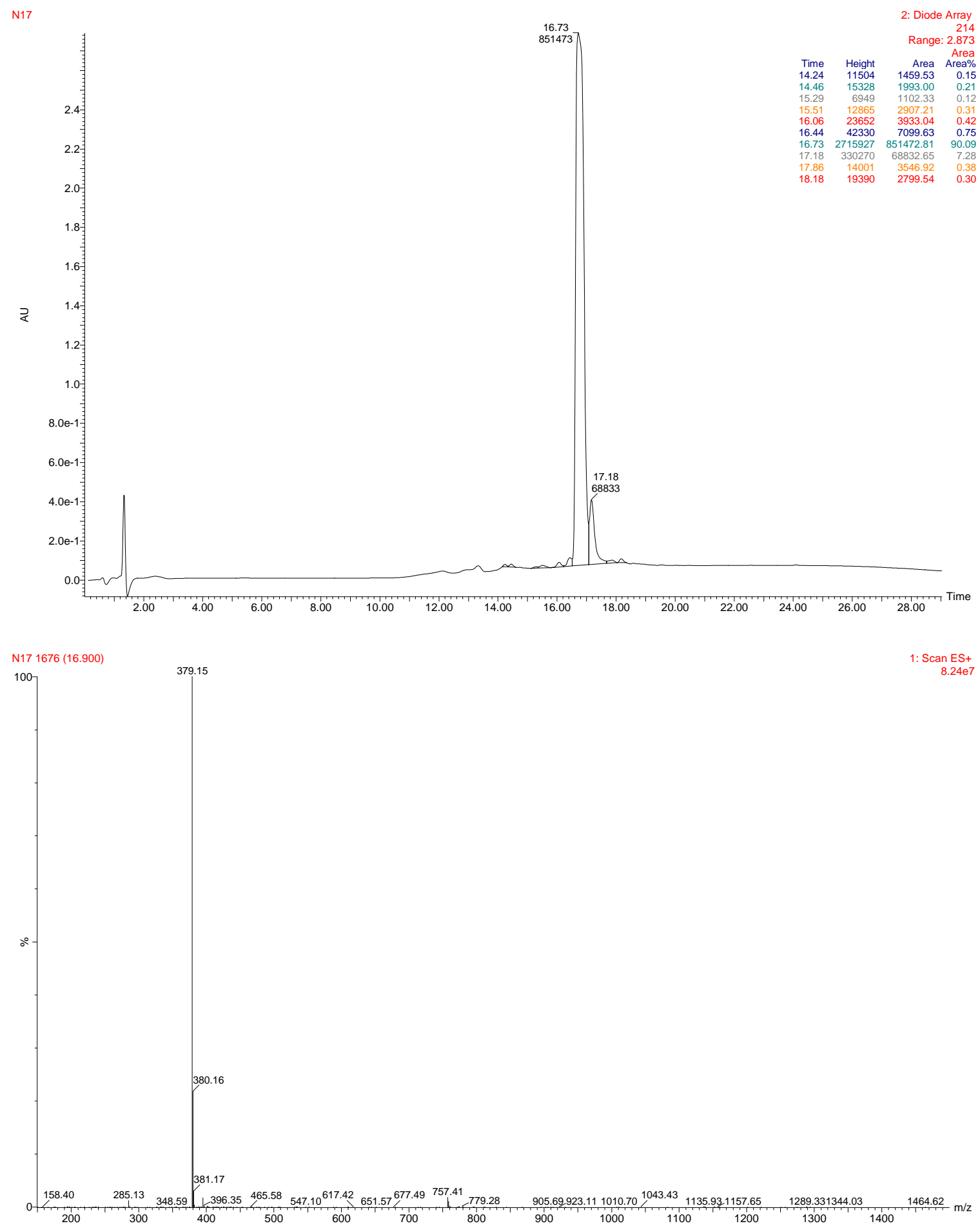

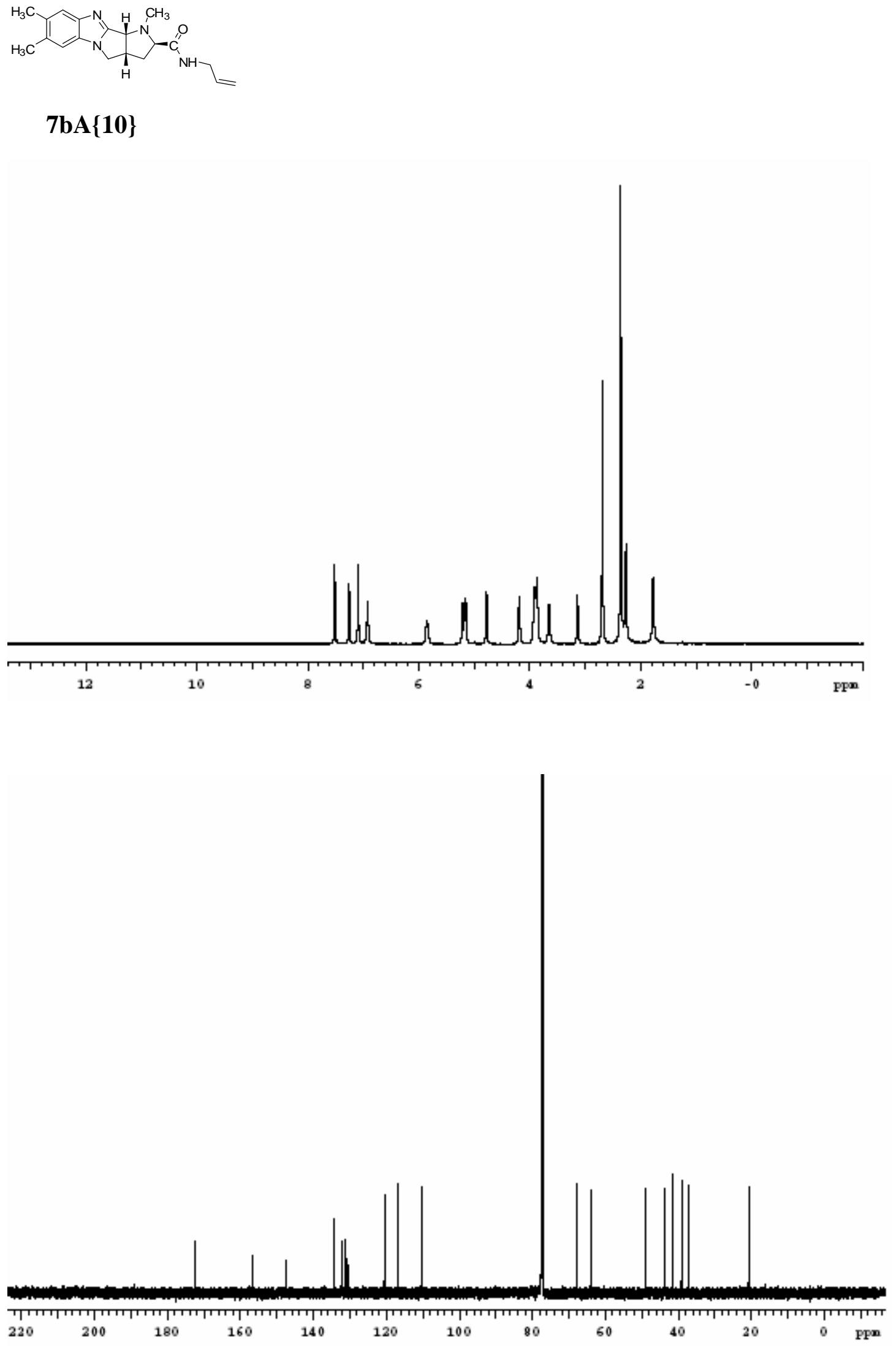

S73 


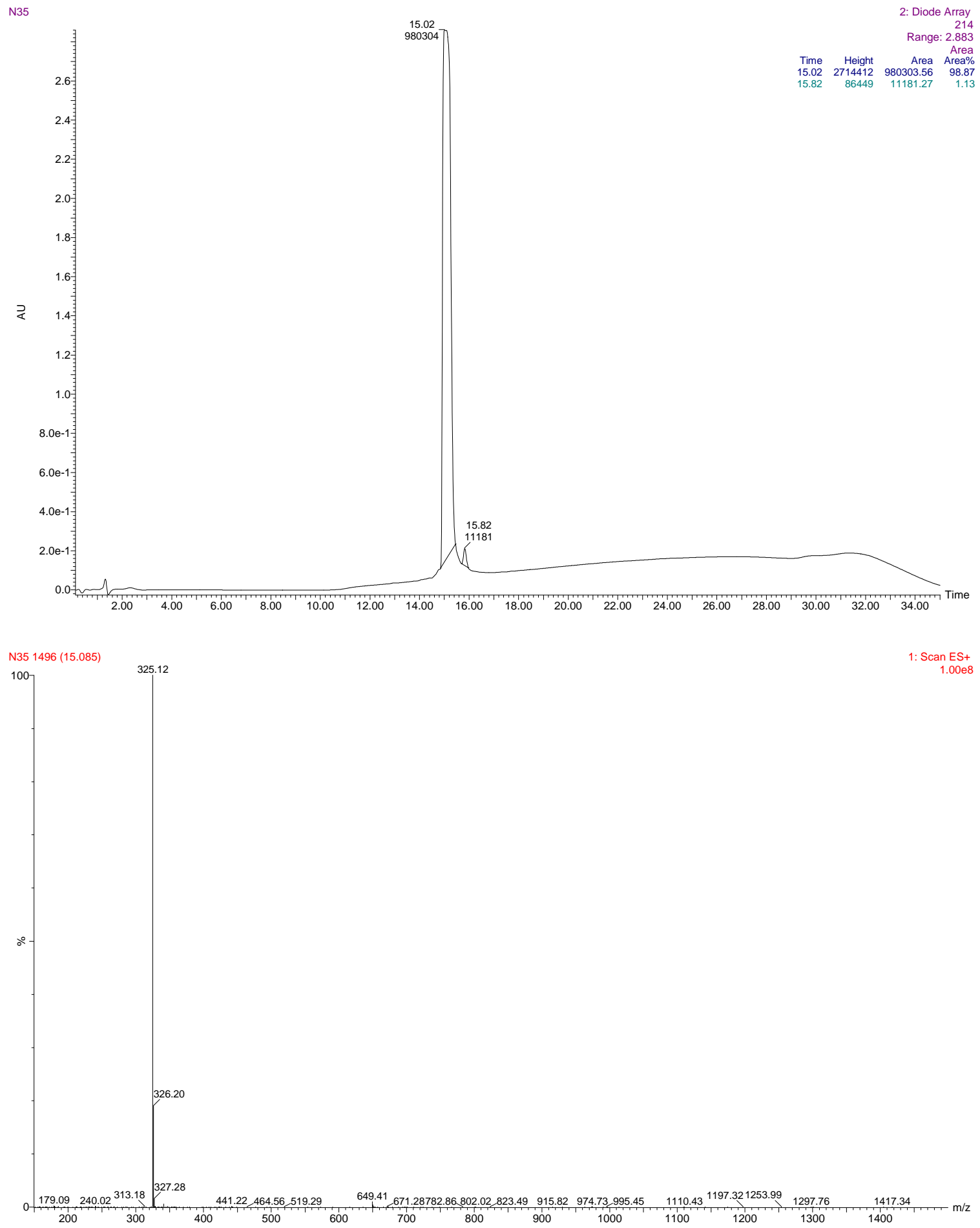




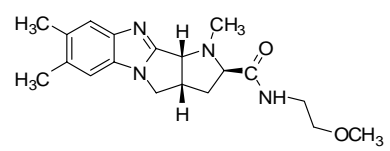

7bA $\{11\}$
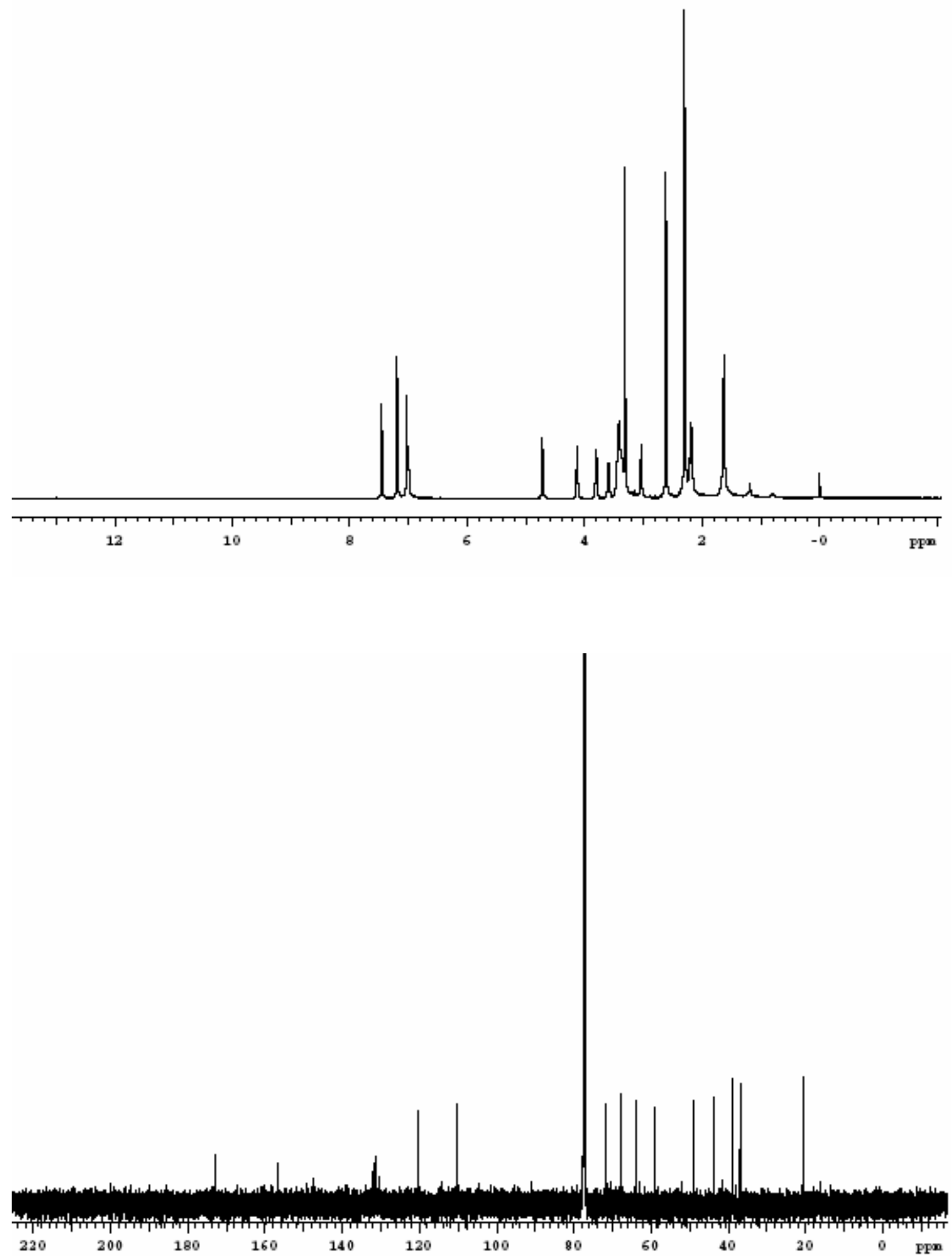


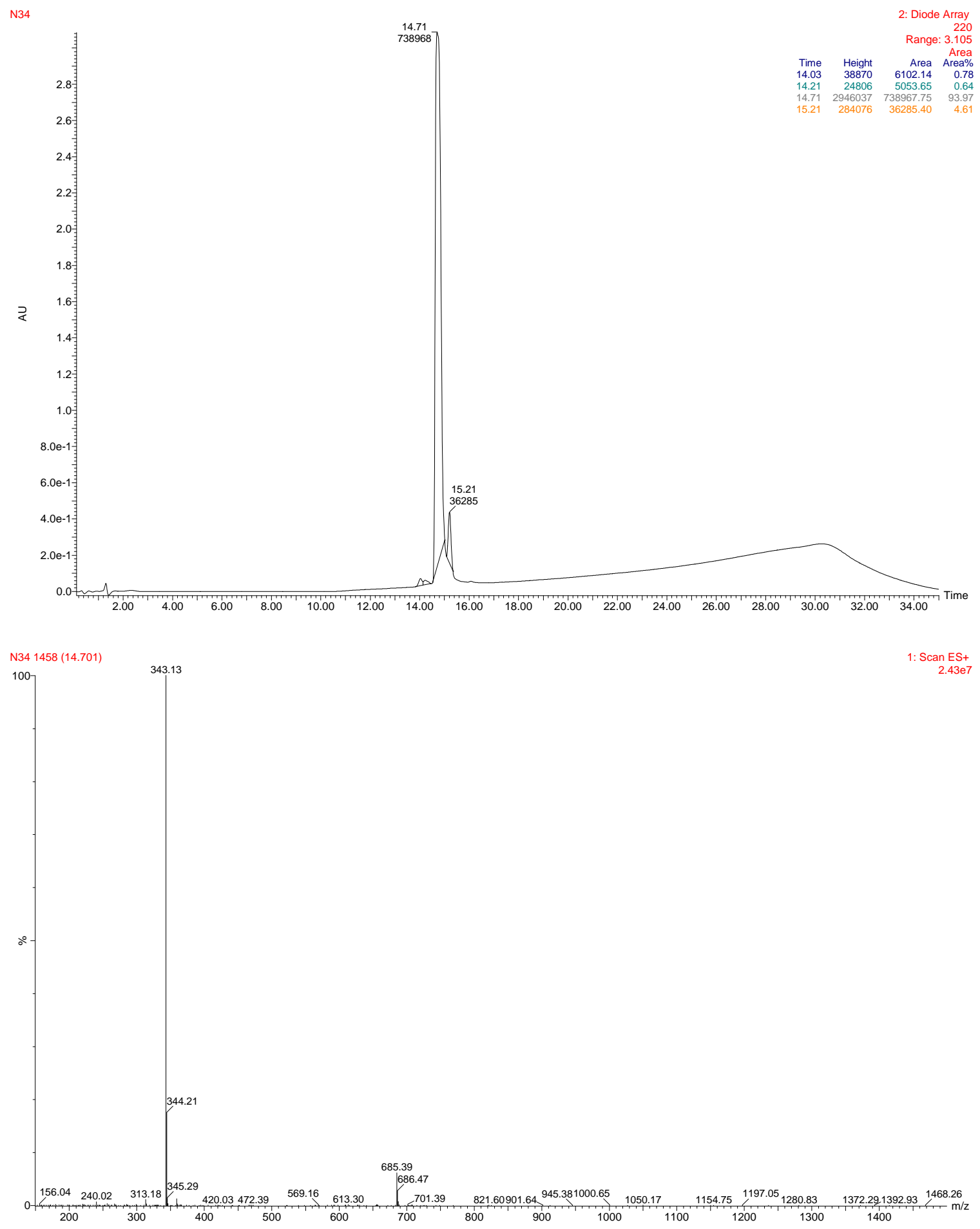




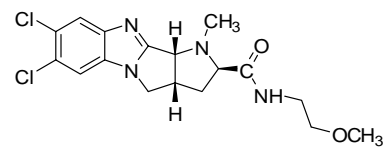

7cA $\{11\}$
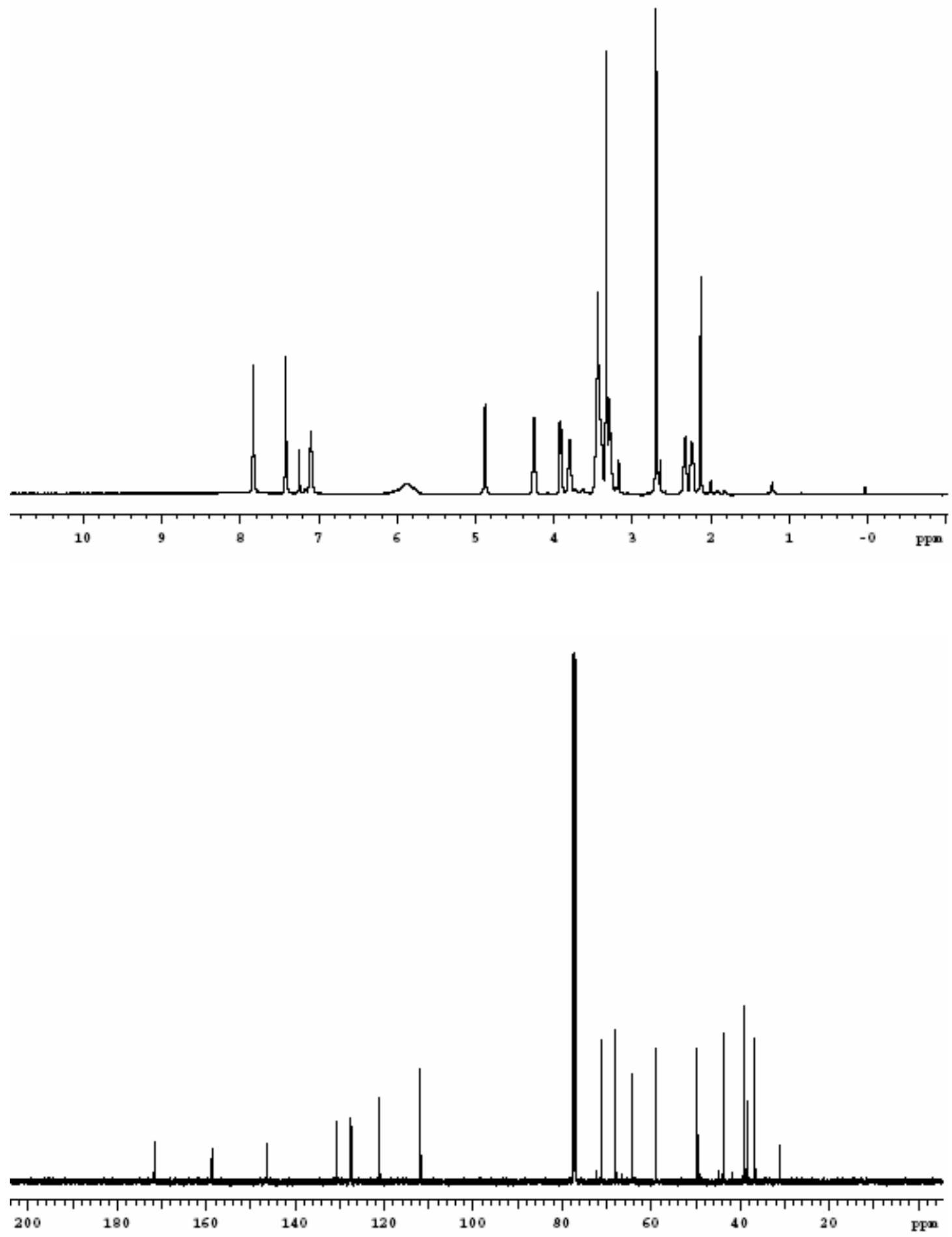

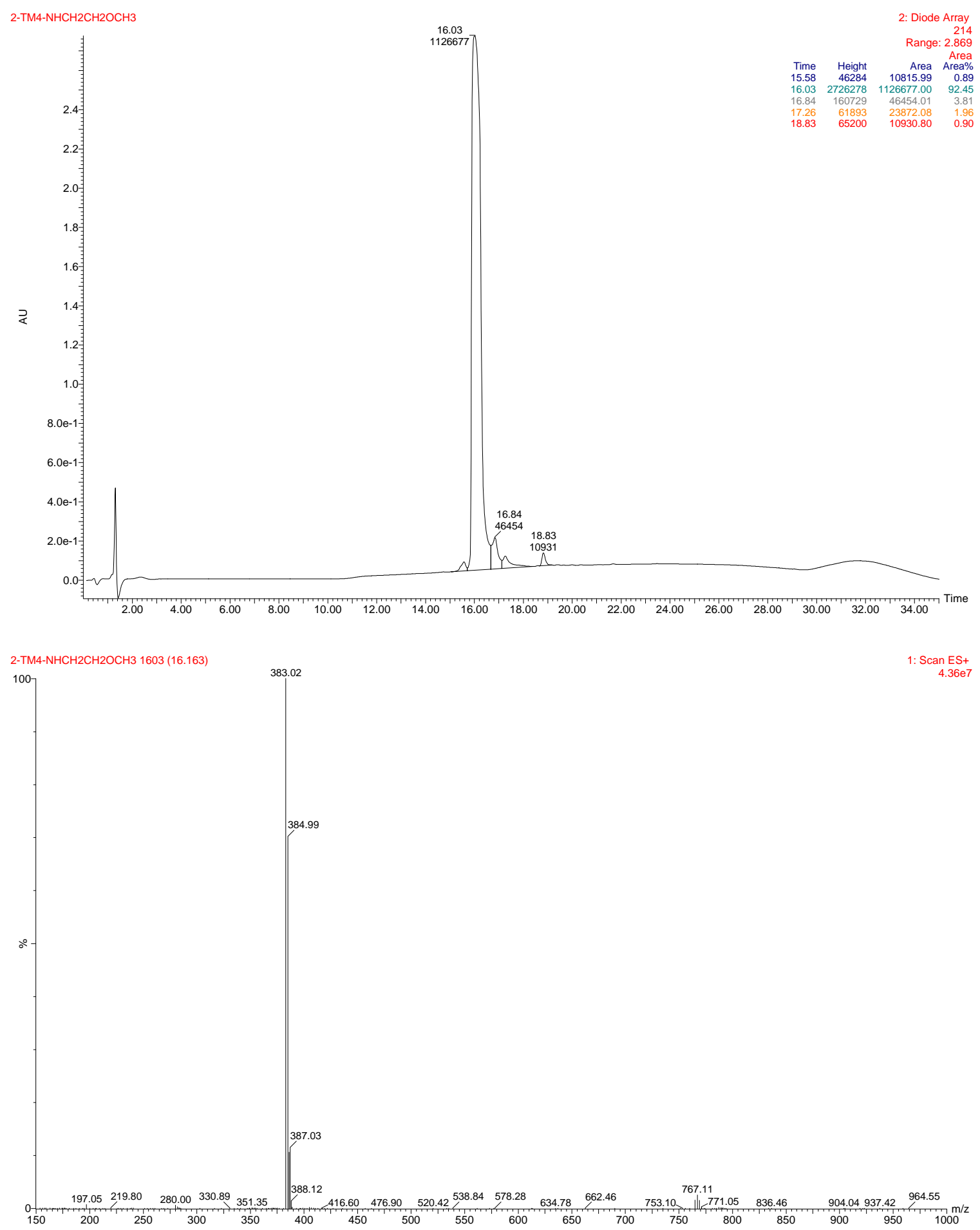


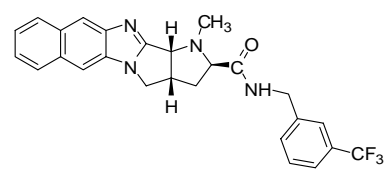

7dA $\{2\}$
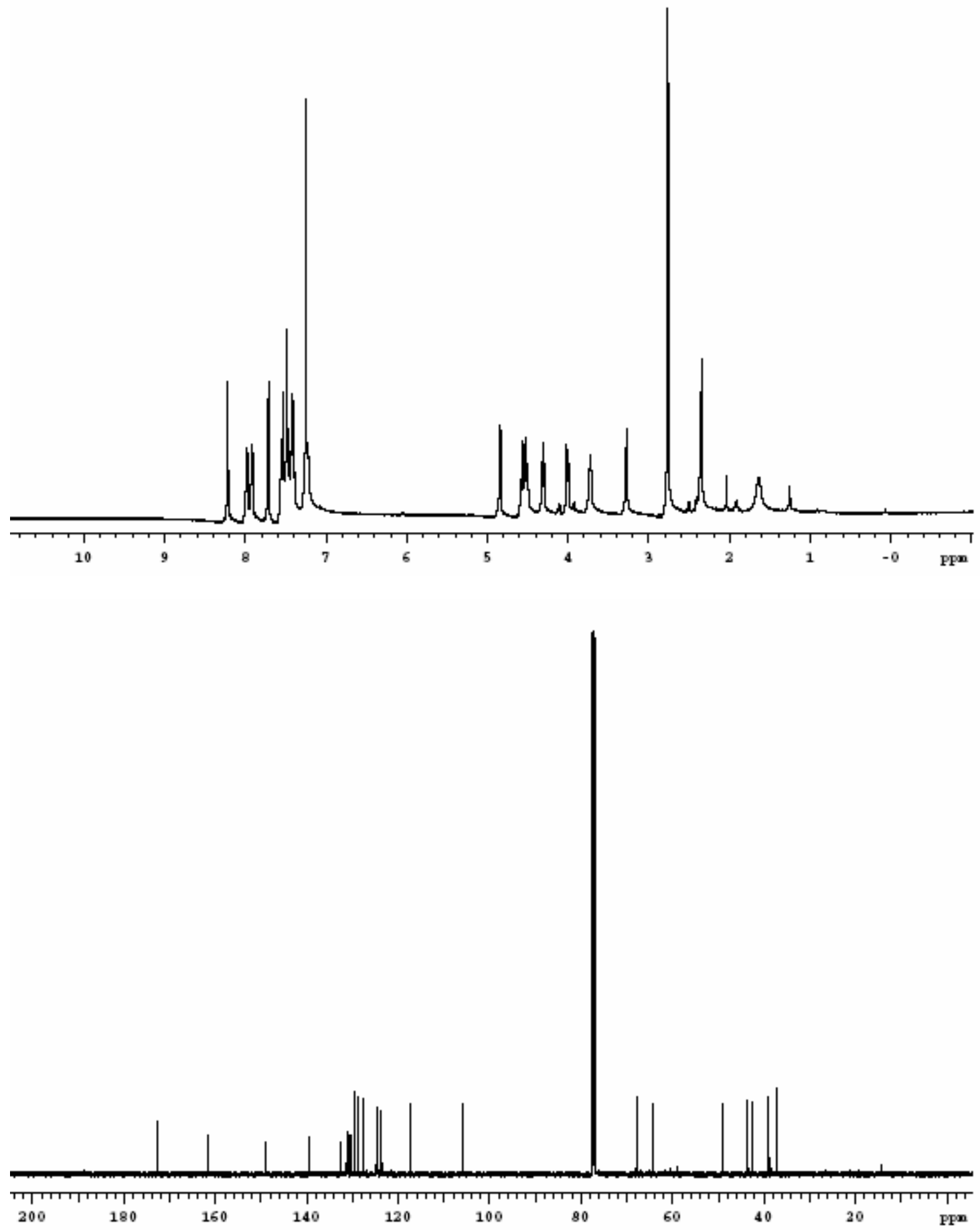


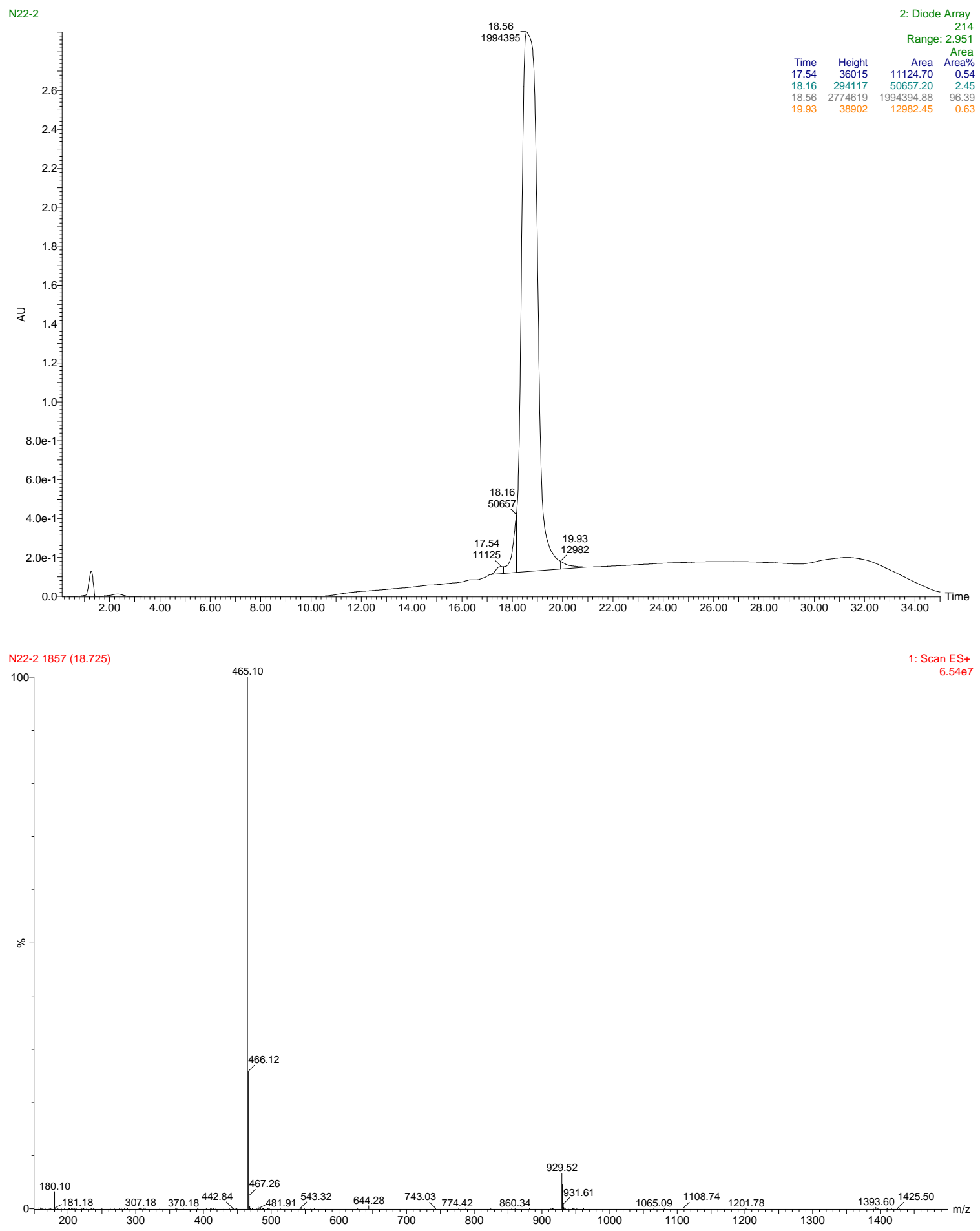




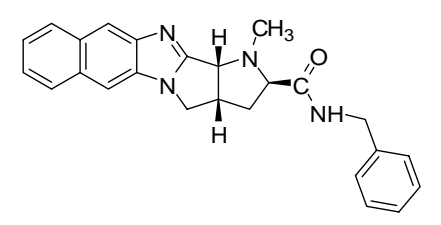

7dA $\{5\}$
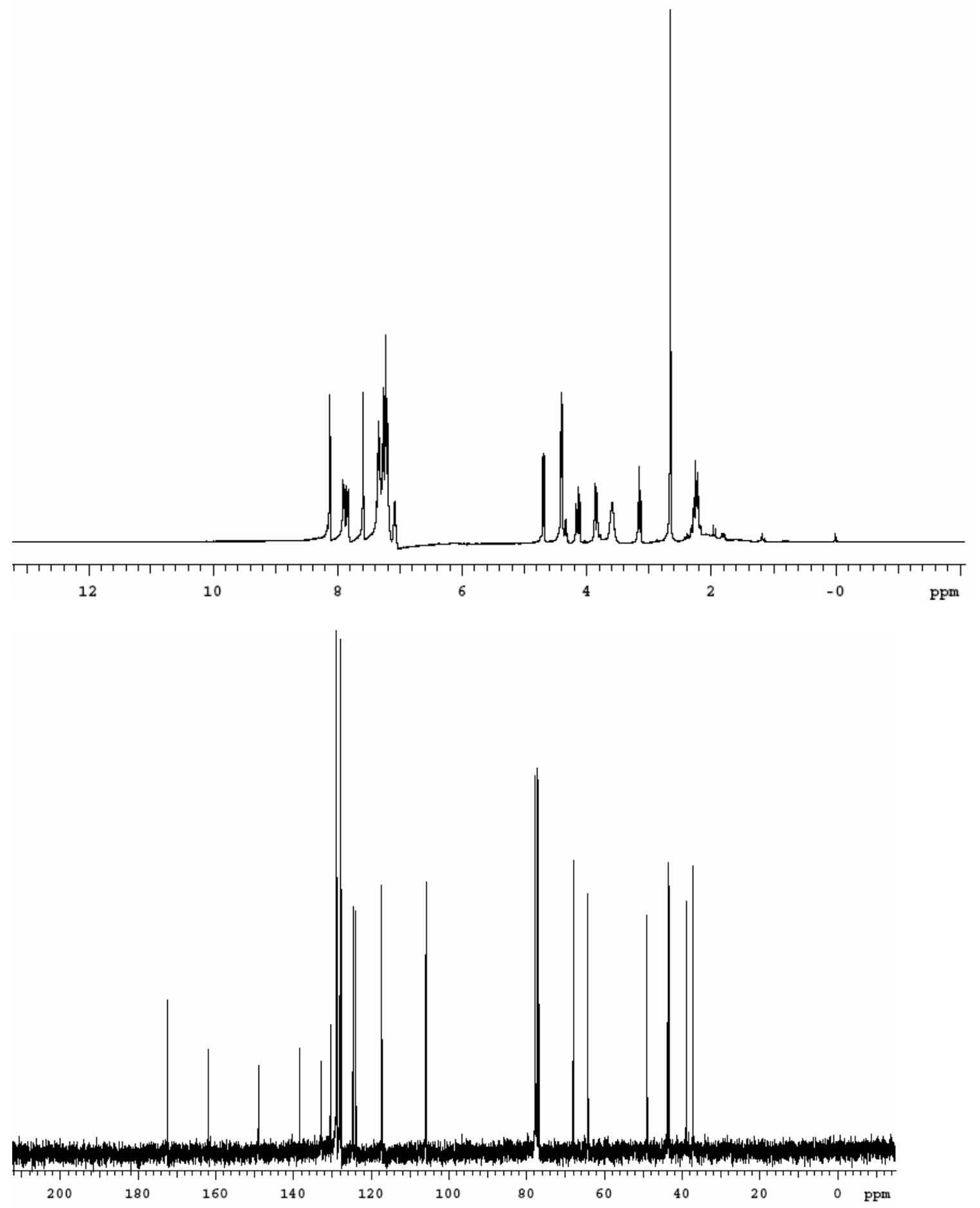


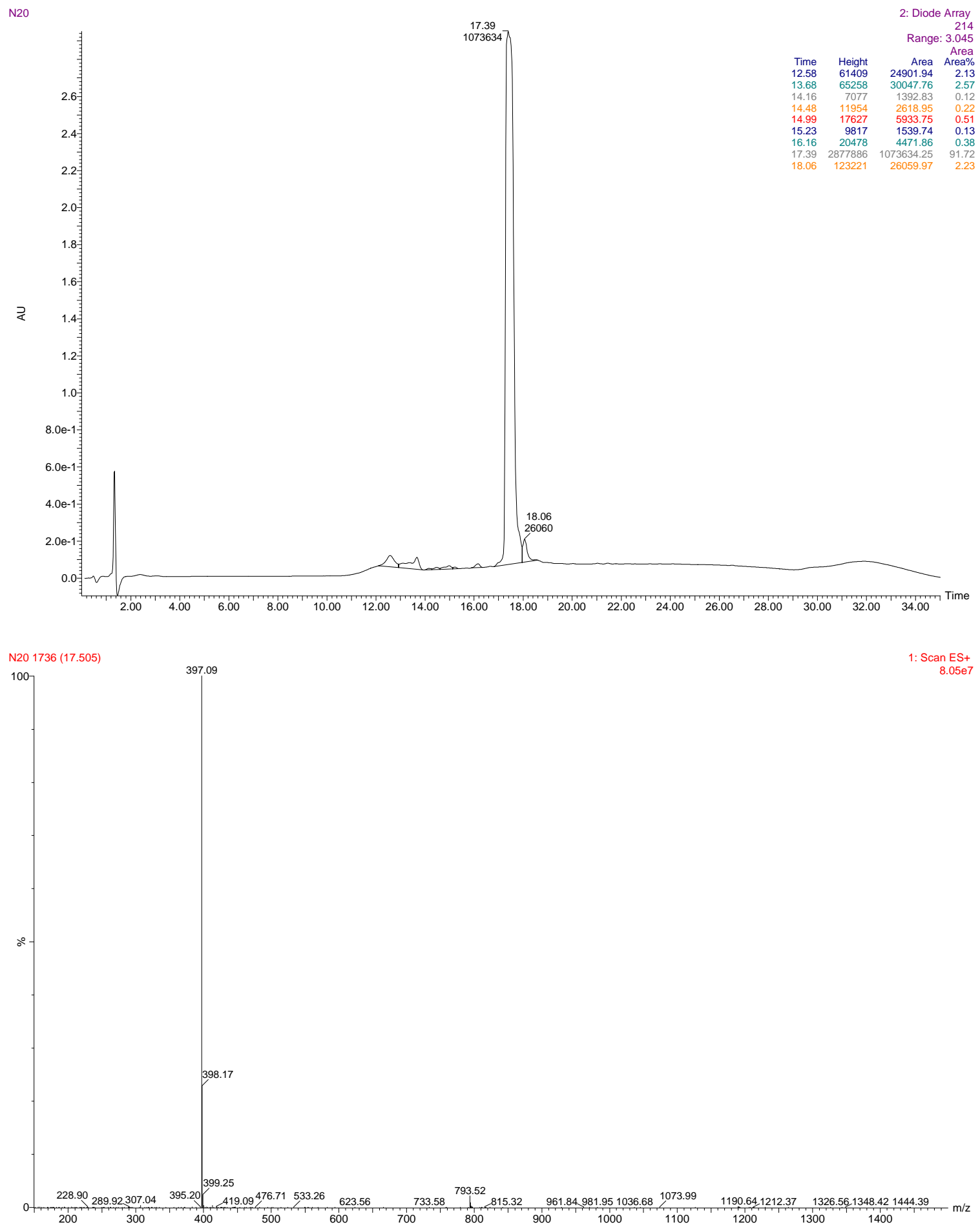

